\title{
Ciclo Económico y Minería del Cobre en Chile ${ }^{1}$
}

\author{
Fernando Fuentes H.2 \\ ILADES- UAH \\ Carlos J. García ${ }^{2}$ \\ ILADES- UAH
}

\begin{abstract}
Resumen:
El presente artículo endogeniza la oferta de cobre, incorporando la demanda por insumos del sector minero correspondiente a otros bienes de la economía, en específico bienes intermedios, y a energía, en un modelo macroeconómico estándar de equilibrio general (DSGE) para una muestra 2003-2013. La estimación del modelo revela que un aumento de 1\% del precio del cobre causa un aumento de 0,16\% en el PIB en cinco años. La principal contribución del estudio es mostrar que si se considera al sector minero integrado al resto de la economía, en vez de suponer que es un enclave, como usualmente se hace, los efectos del precio del cobre sobre la economía chilena por lo menos se duplican.
\end{abstract}

Keywords: Business cycle, Copper-mining industry, Copper price, Bayesian econometrics, DSGE models.

JEL: E17, E27, E37, L72

Septiembre, 2014

\footnotetext{
${ }^{1}$ Se agraden los comentarios de José Tomás Morel, Osvaldo Urzúa y Jorge Cantallopts. El presente estudio fue financiado por el Consejo Minero de Chile. No obstante, la responsabilidad por el contenido recae exclusivamente en los investigadores firmantes.

2 Corresponding authors. ILADES-Universidad Alberto Hurtado, Erasmo Escala 1835, Santiago, Chile. Tel: 56-2-8897366. Fax: 56-2-6920303.E-mail: cgarcia@uahurtado.cl; ffuentes@uahurtado.cl..
} 
I. INTRODUCCIÓN................................................... 2

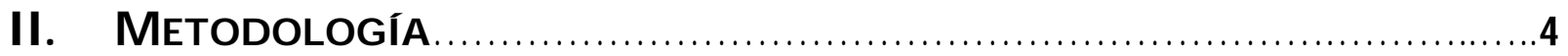

III. REVISIÓN LITERATURA ESPECIALIZADA............................... 9

III.1 LITERATURA NACIONAL............................................. 9

III.2 LITERATURA INTERNACIONAL....................................12

IV. Sector Minero y Ciclo Económico Chileno..............................17

V. Modelo Macroeconómico y Rol del Sector Minero....................30

VI. Estimación Del Modelo: Resultados Principales.....................35

VI.1 RESULTADO ESTIMACIÓN DEL MODELO DSGE........................ 35

VI.2 EFECTO DEL SECTOR MINERO.........................................39

VI.3 CONTRIbUCión DEL SECTOR Minero AL CRECIMIENTO..................43

VI.4 ANÁLISIS DE ESCENARIOS..........................................45

VII. CONCLUSIONES.........................................................49

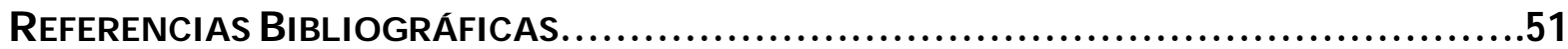

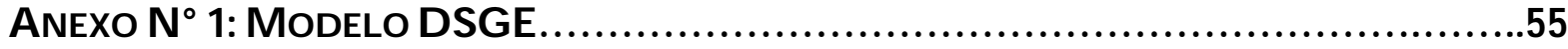

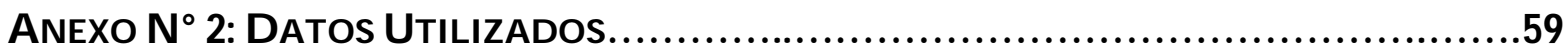

ANEXo N $^{\circ}$ 3: CONVERGENCIA Y PARÁMETROS Estimados Del MOdelo DSGE........62

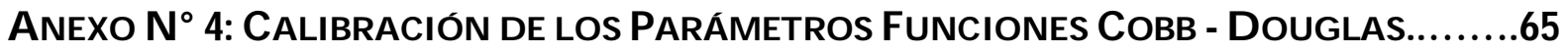




\section{INTRODUCCIÓN}

En Chile el sector minero ha jugado un rol destacado en el desarrollo económico, mostrando importantes tasas de inversión y crecimiento en las últimas décadas. No obstante este hecho que parece evidente, existen pocos estudios que hayan mostrado el impacto real de la minería en el crecimiento del país, y en otras variables de naturaleza macroeconómica que circunscriben dicho crecimiento.

Esta carencia de antecedentes fundados sobre la magnitud de la relación entre minería y desarrollo en nuestro país, podría no ser muy relevante si es que existiera un consenso amplio y claro respecto a los beneficios para Chile y sus habitantes de un pujante sector minero. Sin embargo, se han escuchado en los últimos tiempos voces críticas al tratamiento de la inversión extranjera en Chile en el mencionado sector, llegando incluso a indicarse que los únicos beneficiarios serían los propios inversionistas que rentarían de sobremanera a partir de un bien que pertenecería en rigor a todos los chilenos.

Opiniones como las indicadas podrían en el futuro decantar en posturas políticas que claramente perjudicarían al sector, ya que la convicción de la existencia de inversiones que no ayudan al país y que sólo generan rentas para sus ejecutores es, de facto, un llamado a limitar su participación en Chile.

Unido a este ambiente de cuestionamiento respecto a la relevancia del sector minero y a los beneficios que los chilenos obtienen de su crecimiento, está el hecho que en los últimos años los costos energéticos se han incrementado considerablemente, e incluso se vislumbra la posibilidad de que en el mediano plazo pudiera no existir energía disponible en el mercado para emprender grandes inversiones mineras. Naturalmente, en el contexto descrito si lo que se busca es fomentar futuras inversiones, el primer paso es que la sociedad tenga la convicción de que el desarrollo del sector es positivo para los habitantes de Chile.

En el marco de lo señalado, parece pertinente complementar el conocimiento disponible respecto al verdadero rol del sector minero en el desarrollo de país, cuantificando su contribución a la economía. Para este fin se utilizará un modelo macroeconómico que permite identificar el impacto de la evolución del mencionado sector en las variables más importantes asociadas al desarrollo del país. 
Consistente con lo expuesto, el objetivo principal de este estudio es medir la contribución del sector minero en la economía Chilena a través de la construcción y estimación de un modelo macroeconómico estándar, que explicite las conexiones del sector minero con el resto de los sectores de la economía. El mencionado modelo tiene la virtud de permitir no sólo estimar la contribución del sector minero a la economía en un momento del tiempo, la que se podría cuantificar en base a la matriz insumo producto, sino también medir el aporte de este sector a través del tiempo.

Además, en el marco de la modelación indicada, es posible establecer escenarios alternativos en la producción del sector minero, y cuantificar los efectos de dichos escenarios (respecto de un escenario base) en el resto de la economía. Estos efectos se pueden calcular como cambios en las diversas variables relevantes, entre ellas: el crecimiento en otros sectores productivos, los agregados macroeconómicos (consumo, inversión, empleo, etc.), los ingresos del gobierno provenientes del cobre (y su efecto en la deuda pública y en el gasto fiscal) y el financiamiento de la cuenta corriente (exportaciones y flujo de la inversión extranjera hacia el país).

El estudio se estructurará del siguiente modo. El capítulo II entregará las características estilizadas de la metodología que se empleará para efectos de medir el impacto del sector minero en la economía. El capítulo III realizará una breve reseña de la literatura nacional e internacional relevante. El capítulo IV entregará una primera aproximación a la relación entre el sector minero y el ciclo económico chileno. El capítulo $\mathrm{V}$ describirá la manera en que el sector minero se incorpora al modelo macroeconómico DSGE. Por su parte, el capítulo VI presenta los resultados principales de la estimación de modelo, para terminar con una breve reseña de las conclusiones más importantes de la investigación realizada. 


\section{METODOLOGÍA}

El presente capítulo entregará una primera aproximación a la metodología que se empleará para efectos de medir el impacto del sector minero en la economía del país ${ }^{3}$. Con esta perspectiva, cabe destacar que la conexión del sector minero con el resto de la economía se obtiene modelando aspectos claves de la economía chilena, como el consumo privado, la inversión privada, sectores productivos diferentes al minero, la inflación, el desempleo, la política económica y el sector externo (cuenta corriente). A continuación se presenta un resumen general de la estructura conceptual del modelo, la cual se sintetiza en el Gráfico 1 que se expone más adelante ${ }^{4}$.

\section{SECTOR MiNERO}

En general, la inclusión del sector minero en la modelación macro en Chile ha sido extremadamente simple, de hecho la mayoría de las veces se ha supuesto una oferta de cobre dada 0 exógena, dejando toda la volatilidad de los ingresos de este sector a variaciones en el precio del cobre. Como alternativa a ello, el en el presente estudio se modelará con detalle el proceso productivo del sector minero, de modo de cumplir con el objetivo general del trabajo. En este marco se considerará que los principales insumos del sector son capital, energía y mineral, por lo cual se deberá modelar tanto el proceso de inversión y la demanda por energía, como las expectativas sobre la ley del mineral. Así, se podrá definir con claridad los determinantes fundamentales de la producción del sector, y con ello establecer las razones del crecimiento del sector en el tiempo.

\section{Otros Sectores Productivos}

Se explicitará el sector de bienes intermedios de la economía que puede asociarse a los sectores industriales (y sus respectivas exportaciones) y de comercio. También se modelará el sector construcción (inversión en construcción).

\section{CONSUMO}

El consumo se modelará suponiendo la existencia de dos tipos de familias, familias con acceso al mercado del crédito (definidos como optimizadores) y familias que dependen sólo de su ingreso

\footnotetext{
3 Más adelante, en el capítulo $\mathrm{V}$ y en el anexo $\mathrm{N}^{\circ} 2$, se explicitarán las ecuaciones específicas empleadas.

4 En el Gráfico 1 el sector minero debe entenderse induido en el "Sector Externo".
} 
laboral para financiar su consumo (definidos como restringidos). Esto permite una modelación realista del consumo agregado, que es aproximadamente un $60 \%$ del PIB.

\section{INVERSIÓN}

La inversión privada se modelará suponiendo dos tipos de firmas, aquellas con acceso al mercado financiero, es decir, firmas que podrán tomar sus decisiones de inversión en función del valor esperado de sus ingresos futuros, y firmas restringidas, que podrán invertir dependiendo sólo de su flujo de caja. Se considerará asimismo la inversión en construcción. Esto permite una modelación realista de la inversión agregada, que es aproximadamente un 20\% del PIB.

\section{INFLACIÓN DE PRECIOS Y SAIARIOS}

Los precios de la economía (IPC) y los salarios se supondrán que reaccionan lentamente al ciclo económico, con esto se captura un hecho estilizado de la economía chilena en que los precios de los bienes y los salarios nominales se mantienen a nivel agregado rígidos entre tres y cuatro trimestres.

\section{CUENTA CORRIENTE}

Las exportaciones se separarán en cobre y no cobre, de manera similar se considerará las importaciones de petróleo y no petróleo. Además se modelará las remesas y las transferencias al exterior.

\section{POLÍTICA EOONÓMICA}

Se modelará el Banco Central, donde la tasa de política monetaria (TPM) depende de la inflación y el PIB. En cambio, el gobierno seguirá en el largo plazo una regla estructural basada en los ingresos estructurales (PIB y precio del Cobre). En este contexto, se permitirán desviaciones de esa regla fiscal en el corto plazo a través de cambio en la deuda pública. 


\section{Gráfico 1}

\section{Esquema del Modelo de Consistencia}

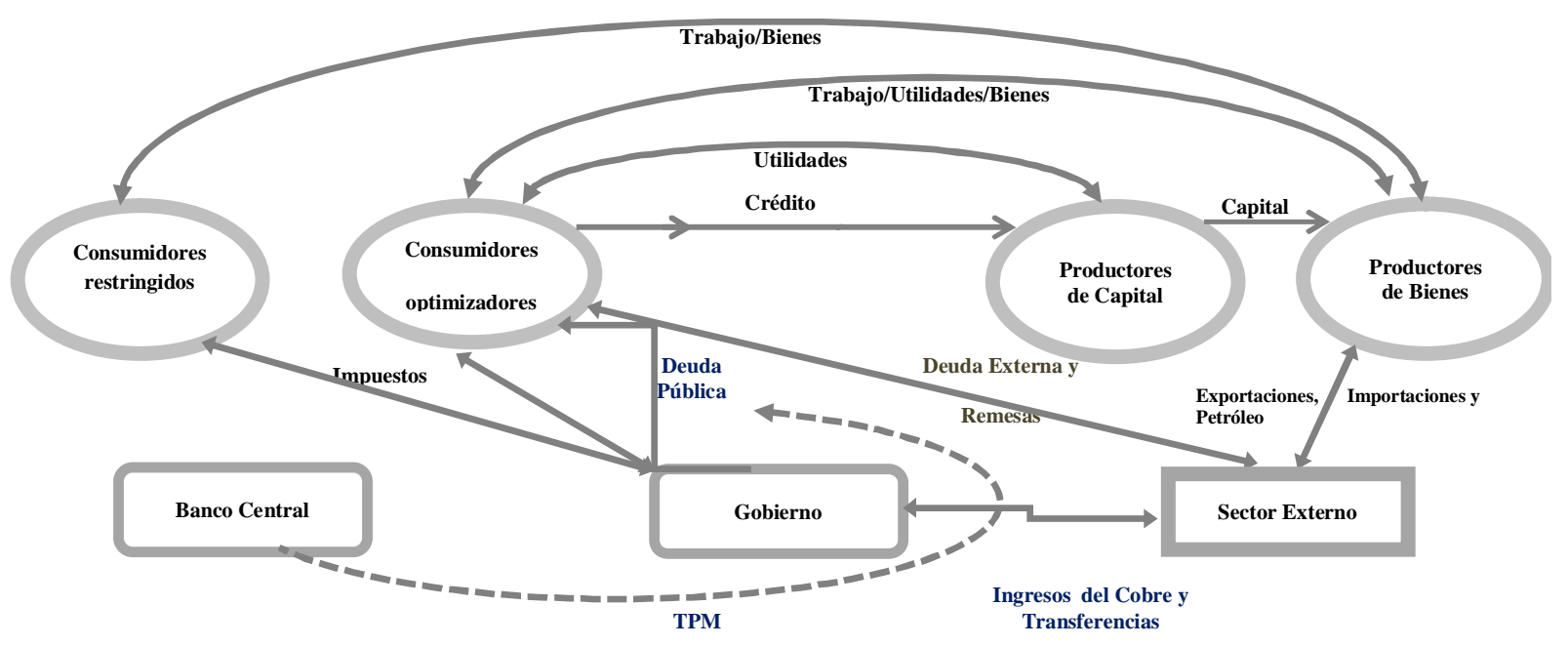

\section{ASPECTOS DeSTACADOS DE LA MODELACIÓN}

Los modelos que describen con detalle las principales conexiones entre sectores y agentes económicos se conocen por modelos DSGE5 . Son modelos que usan un enfoque de equilibrio general (es decir, de consistencia) para poner en su interior el conjunto de relaciones económicas más estándares en macroeconomía. Se puede decir que los DSGE representan el lenguaje común entre los macroeconomistas en los últimos años, después de haberse reemplazando el antiguo esquema IS - LM. El enfoque DSGE es una mezcla entre los modelos IS - LM y los $\mathrm{RBC}^{6}$, es decir, hereda el realismo del enfoque IS - LM con la consistencia teórica de los RBC o modelos neo-clásicos introducidos a principio de los ochenta del siglo pasado.

El Cuadro 1 presentado más adelante resume las fortalezas y debilidades de sucesivas generaciones de modelos macroeconómicos usados para analizar las fluctuaciones económicas. Dentro de este conjunto de enfoques, la nueva generación de modelos de equilibrio general DSGE corresponde como se ha dicho a una combinación entre los modelos keynesianos y los de ciclos reales (ó $\mathrm{RBC}$ ).

\footnotetext{
${ }^{5}$ Dynamic Stochastic General Equilibrium

${ }^{6}$ Real Business Cycle.
} 
Los modelos keynesianos tradicionales si bien están muy orientados al análisis de políticas macroeconómicas, tienen importantes debilidades en especial a lo que se refiere a la formación de expectativas y la modelación de la oferta de la economía. El supuesto de expectativas mecánicas (adaptativas) introduce falencias relevantes cuando se simulan nuevas políticas, porque los cambios de política alteran las expectativas y con ellas los valores de los parámetros reducidos del modelo.

En cambio, los modelos de ciclos reales o RBC tienen sólidos fundamentos teóricos, en especial de la oferta, pero con escasa utilidad para el análisis de política, especialmente en países en desarrollo por los supuestos excesivamente simplificatorios sobre la estructura de la economía (precios y salarios flexibles, y equivalencia Ricardiana ${ }^{7}$ entre los más importantes). Con lo cual tanto la política fiscal como la monetaria no tienen efectos reales, supuesto altamente discutible a la hora de elegir un modelo para analizar la estabilización de corto y mediano plazo.

Los DSGE tienen características largamente anheladas por los hacedores de política (policy makers); posee una estructura donde se describe con detalle la demanda y la oferta de distintos sectores económicos, y a su vez es útil como sus antecesores para analizar diferentes políticas, shocks y escenarios alternativos. Además, para algunas variables (inflación y crecimiento) se obtienen proyecciones tan buenas como las realizadas por los modelos de series de tiempo (VAR, ARIMA, etc.). Esto permite juntar en una sola metodología buenas explicaciones con proyecciones razonables, con lo cual incrementa las posibilidades de realizar políticas adecuadas tanto del punto de vista monetario como fiscal.

En términos generales, el DSGE que se utilizará en el presente estudio será un modelo de equilibrio general muy usado para el análisis de corto y mediano plazo de las fluctuaciones cíclicas de la economía en muchos bancos centrales (Canadá, Japón, la Reserva Federal de los Estados Unidos, etc.) y oficinas gubernamentales. El modelo está basado en el trabajo seminal desarrollado por los economistas del Banco Central Europeo (ECB) Smets y Wouters $(2003,2007)$, pero también se fundamenta en el trabajo de Gertler y Kiyotaki (2010), Galí (2008), Galí y Gertler (2007), Galí et al (2007), Ercer et al (2006), Christiano et al (2005), Galí et al (2004), y Woodford (2003). Los detalles sobre la estimación de estos modelos pueden encontrarse en Dejong y Dave (2011) y An y Schorheide (2007).

Los modelos DSGE comparten algunas similitudes básicas con otros modelos de equilibrio general; los modelos computables de equilibrio general ó CGE. Sin embargo, estos últimos no han

7 Se entiende por equivalencia ricardiana la propiedad de inefectividad de la política fiscal, en especial de cambios en los impuestos sobre el consumo. 
sido usados comúnmente para el análisis macroeconómico por adolecer de una serie problemas relacionados con la formación de expectativas y la falta de un análisis estocásticos y dinámico realista que permita un estudio sensato de los fenómenos macroeconómicos más relevante (inflación, empleo, crecimiento, competitividad, etc.). Este tipo de modelos no pertenecen a la tradición de modelos macro propiamente tal y ellos se han dejado para el análisis del comercio exterior entre países (acuerdos comerciales) y temas ambientales.

\section{Cuadro 1}

\section{Resumen de las debilidades y fortalezas de los Modelos Macro(*)}

\begin{tabular}{|c|c|c|c|c|}
\hline \multirow[t]{4}{*}{ Modelos } & Keynesiano & Keynesiano & Modelo & Modelo \\
\hline & Expectativas & Expectativas & Ciclos & Estocástico de \\
\hline & Adaptativas & Racionales & Reales RBC & Equilibrio \\
\hline & & & & General DSGE \\
\hline \multirow[t]{5}{*}{ Fortalezas } & Permiten analizar & Generan dinámicas & Sólidos fundamentos & Integran demanda y \\
\hline & de manera unificada & más realista & teóricos que mejoran la & oferta a través \\
\hline & impactos de política & para analizar shocks & modelación de la oferta & de fundamentos microeconómicos \\
\hline & y otros shocks cíclicos sobre & & agregada y permite cálculo & Incorpora precios y \\
\hline & la economía & & de bienestar & salarios rígidos \\
\hline \multirow[t]{6}{*}{ Débilidades } & Expectativas adpatativas & Ausencia de fundamentos & Supuesto de precios flexibles & Factibles de construir \\
\hline & pueden provocar que los & teóricos sólidos & limita el análisis de & por avances computacionales \\
\hline & policy makers & para analizar el efecto de shocks & política macroeconómica & \\
\hline & consistentemente & sobre la oferta agregada & & \\
\hline & caigan en errores y tengan sesgo & & & \\
\hline & hacia políticas expansivas & & & \\
\hline
\end{tabular}

(*)Los modelos de series de tiempo (ARIMA, VAR, etc.) son excluidos de esta tabla. Sus fortalezas son bien conocidas, precisión en las proyecciones pero con una limitada capacidad de interpretación estructural de los resultados.

Un último aspecto que es relevante destacar, es que en Chile los modelos DSGE se han desarrollado desde mediados de la década pasada. Las primeras versiones de DSGE pueden ser encontradas en, Soto y Medina (2006, 2007), Caputo et al (2007), Céspedes et al. (2010), García et al. (2011a,b) y García et al (2013), García y González (2013a,b) quienes han utilizado estos modelos tanto para hacer proyecciones como análisis económico. En el Banco Central de Chile algunos de estos estudios dieron nacimiento al llamado MAS, modelo DSGE que complementa las proyecciones que se realizan con múltiples modelos de series de tiempo univariados, VAR y sus derivados, y el MEP, modelo Neo-Keynesiano reducido (Banco Central de Chile, 2003). Además estos modelos han servido para que el Consejo de Banco y sus economistas principales definan los escenarios que se publican periódicamente en el informe de política monetaria de Banco Central de Chile, más conocido por IPoM. 


\section{REVISIÓN LITERATURA ESPECIALIZADA}

El presente capítulo entrega una breve reseña de la literatura nacional e internacional respecto del tema minero en general, y su eventual impacto en la economía a través de sus variables agregadas fundamentales. Para estos efectos, con fines de presentación, se ha incluido los antecedentes referidos a la economía chilena en primer lugar, para luego concentrarse en las referencias enfocadas en otros países.

\section{III.1 LITERATURA NACIONAL}

La literatura nacional sobre el sector minero establece diversas perspectivas para analizar el sector, desde la simple descripción o entrega de antecedentes hasta aquellos estudios que intentan establecer relaciones más complejas entre la minería y la economía en general. Sin embargo, más allá de esta variabilidad de enfoques y de la existencia de tratativas por medir el impacto de este sector en la economía a través del uso de la matriz insumo - producto, no existen antecedentes de mediciones o proyecciones llevadas a cabo desde una óptica de análisis dinámico basado en modelos macroeconómicos de equilibrio general, que representa el foco esencial del presente estudio. Teniendo en mente lo indicado, a continuación se entregará una breve reseña algunos trabajos seleccionados que sirven de contexto para la investigación que se desarrollará en los siguientes capítulos.

En el ámbito descriptivo, destaca el Anuario de la Minería de Chile del SERNAGEOMIN que detalla el volumen de la producción, el nivel de exportaciones, los precios, la relación precios/ producción y el consumo energético de la gran minería. Asimismo, identifica subgrupos de datos por recurso minero y por región, y presenta estadísticas de operación y gestión minera, contexto en el que desarrolla temas de seguridad minera, concesiones y medio ambiente. Estos datos son evidentemente relevantes para hacer un estudio de impacto económico del sector minero, no obstante representa una serie muy corta para realizar un exhaustivo análisis (2008-2012). En relación a la vinculación y/ o dependencia que existe entre el sector minero y el energético, Jiménez, S. (2013) analiza el impacto que ha generado el atraso de los proyectos energéticos en el sector minero y, a través de dicho sector, en la economía en general, considerando de modo particular el efecto sobre las arcas fiscales. La mencionada autora inicia su estudio haciendo un barrido de datos

\footnotetext{
8 Servicio Nacional de Geología y Minería (2012).
} 
que intentan vincular el PIB con el consumo eléctrico y, además, presenta una serie de inquietudes referidas al incremento en los costos de la minería y su impacto en la competitividad del sector. Por último, esboza posibles soluciones al problema descrito, como por ejemplo la necesidad de aumentar la oferta de energía de base (hidroeléctrica y termoeléctrica), la facilitación de trámites y el freno al activismo judicial, mediante la difusión de información transparente y objetiva en materia energética, y la estandarización de metodologías de impacto ambiental, en un contexto de fomento a la participación ciudadana.

Desde otra perspectiva, destaca un área temática referida a la minería y su impacto económico, con trabajos dedicados a analizar, por ejemplo, los costos de la minería desde la óptica de sus dimensiones ambientales. Es así como, Borregaard, N. (2009) analiza los valores de las externalidades económicas asociadas al uso de agua, las emisiones de dióxido de azufre, el riesgo implicado en el transporte de sustancias peligrosas y otros riesgos ambientales. Por su parte, Leal, J. (2000) revisa métodos de valoración contingente, funciones de producción domésticas y precios hedónicos, para efectos de estimar los costos ambientales de la actividad minera, técnicas que hasta ese entonces no habían sido aplicadas al sector minero chileno. Ambos trabajos se focalizan en dimensionar correctamente los costos ambientales de modo de priorizar intervenciones y/o mitigaciones de impactos, elaborar una política medioambiental eficiente y potenciar la gestión ambiental.

Otra agrupación temática la constituyen aquellos estudios que visualizan a la minería como promotora del desarrollo económico. Trabajos como los de Aroca, P. (2012), De Gregorio, J. (2009) y Álvarez, R. et al (2004) analizan el crecimiento sectorial de la minería, vinculándolo a la ventaja comparativa de Chile en la extracción de minerales y/ o recursos naturales. Estos autores comienzan contextualizando el desarrollo minero en Chile, identificando ciclos y políticas al respecto, y analizando la participación del sector en el PIB. Asimismo, focalizan la relación del sector minero con otros sectores económicos, a través de su efecto multiplicador en la economía. En el contexto indicado, también ponen de manifiesto el grave problema que ocasiona la falta de profesionales en el país, destacando la poca alineación existente entre el sector productivo y las instituciones de formación, la importancia de la institucionalidad y certeza jurídica a nivel país para el desarrollo del sector minero, y el impacto de los factores productivos (como el capital humano y físico) en el crecimiento del sector.

Otras investigaciones se concentran en los impactos económicos sectoriales de la minería. Este es el caso de Aroca, P. (2000), Acevedo, R. et al (2006) y el de COCHILCO (2013) que, en base a una 
matriz de insumo-producto, calculan los multiplicadores del sector minería con respecto a los otros sectores de la economía. Cabe señalar que este impacto es calculado sobre variables como el producto, el empleo y las remuneraciones (o ingresos). Aroca, que focaliza su trabajo en la segunda región, destaca que los multiplicadores captan los efectos directos e indirectos (en otros sectores) de un cambio en una determinada variable, pero no captarían los efectos inducidos en variables que no forman parte de la matriz (el impacto en el empleo, por ejemplo). Debido a lo anterior, considera dos tipos de matrices insumo-producto, una primera que solo considera los efectos directos e indirectos, y una segunda que incluye los efectos inducidos en el empleo. Los resultados encontrados muestran que los efectos multiplicadores son mayores al utilizar el segundo tipo de matriz, y las grandes diferencias se encuentran en aquellos sectores que son intensivos en el uso de mano de obra. Por ejemplo, el mencionado autor encontró, al aplicar el modelo de dos matrices, que el multiplicador de la matriz abierta (sin efectos inducidos) varía entre 1.02 y 1.66 para los 12 sectores de la economía analizados, mientras que para la matriz cerrada varía entre 1.76 y 3.96. Resultados análogos encontró COCHILCO (2012), cuando nivel nacional incluye el efecto del empleo y las remuneraciones. ${ }^{9}$

Soto, R. et al (1998) concentra sus esfuerzos en analizar, para el caso particular de una mina específica instalada en Chile, el impacto de la actividad de dicha mina en tres áreas de la VI región: el efecto empleo (directo e indirecto), el efecto creación de riqueza (valor agregado directo e indirecto) y el efecto de externalidades (positivas y negativas). Estos efectos directos e indirectos son calculados a través de la observación de los diferentes sectores económicos, considerando la creación de riqueza y el empleo generado. En cuanto al impacto de la minería en los ingresos fiscales regionales, los mismos autores anteriores (Aroca y Soto) y Leturia, F. et al (2004) exponen dos facetas del tema analizado: por un lado, los ingresos derivados de los impuestos que van a dar al fisco, los cuales se distribuyen en el país sin criterio de procedencia; y por otro, los ingresos derivados de las patentes mineras que se quedan en la región. En el trabajo de Leturia et al, también se intenta desmitificar los dichos acerca del comportamiento tributario del sector minero en Chile, como por ejemplo, que gozan de un régimen tributario preferente, distinto al del resto de los contribuyentes. En general, los citados trabajos tratan sobre los impactos del sector minero en la comunidad, los cuales se pueden desglosar en dos grandes dimensiones: los planes de beneficio a la comunidad en donde destacan fundaciones, proyectos educativos y subsidios habitacionales para

\footnotetext{
9 En este contexto es relevante destacar que el modelo dinámico de equilibrio general que será usado en el presente trabajo permite visual izar directamente (como resultado de la estimación) el efecto de variaciones de las variables fundamentales en magnitudes que no forman parte de la matriz insumo producto, como el empleo.
} 
los trabajadores; y los efectos negativos para la salud de la población, en donde se mencionan las enfermedades respiratorias derivadas de la explotación minera.

COCHILCO (2012) también analiza las bases del éxito minero vinculado a la gestión jurídicainstitucional del país, su impacto económico y social a través de los aportes fiscales que contribuyen al desarrollo de otros sectores como la salud y la educación, y la competitividad del sector que ha ido disminuyendo dado los crecientes costos energéticos y falta de certeza jurídica en la aprobación de algunos proyectos. Asimismo, muestra el impacto de las empresas mineras en los proveedores locales, evidenciando que gran parte de la economía regional minera está ligada a esta relación.

Meller (2013) presenta un modelo econométrico que relaciona en una ecuación reducida el crecimiento de la economía chilena con la actividad minera. Sus resultados indican que cuanto más se expanden las exportaciones de cobre, más crece la economía, refutándose, según este estudio, la hipótesis de enfermedad holandesa que podría haber causado el boom del sector del cobre en la última década.

Tal como fuera indicado, se puede observar que si bien existen esfuerzos por identificar la importancia de la minería en la economía nacional, no se ha estudiado de manera formal las dimensiones dinámicas del problema desde una óptica macroeconómica, incluyendo funciones de impulso respuesta que permitan visualizar el efecto de shocks específicos al equilibrio intertemporal de la economía.

\section{III.2 LITERATURA INTERNACIONAL}

Dado el foco fundamental de la presente investigación, la literatura internacional respecto de la cual se realizará una breve reseña estará referida esencialmente a la explicación de la existencia de dos formas de introducir un commodity minero en un modelo macro estándar (del tipo DSGE).

En primer término cabe destacar que existe una vasta literatura de modelos DSGE para economías pequeñas y abiertas, que analizan los efectos del precio de un commodity minero sobre la economía suponiendo que la producción (oferta) de dicho commodity es exógena. Ejemplos de estos trabajos son Dib (2008), Bems y de Carvalho Filho (2011), Bodenstein, Erceg y Guerrieri (2011), Garcia et al (2011), Lama and Medina (2012), Natal (2012), y Garcia y González (2014). Una perspectiva abstracta similar es también común en los modelos DSGE desarrollados por varios bancos centrales, incluyendo Australia (Jääsleä y Nimark 2008), Canadá (Murchison y Rennison 
2006), Nueva Zelanda (Lees 2009), Chile (Medina y Soto 2007) y España (Andrés, Burriel y Estrada 2006). En resumen, todos los mencionados modelos introducen el valor de la exportación del commodity como un elemento adicional en la balanza de pagos, también llamada en la literatura de modelos DSGE; "restricción de la economía". En consistencia con lo indicado, el enfoque se puede expresar a través de la ecuación (1.1) que representa una típica "restricción de la economía", donde el financiamiento del gasto total $P_{t} C_{t}+P_{t} I_{t}+P_{t} G_{t}$, donde $C_{t}$ corresponde al consumo privado, $I_{t}$ la inversión privada, $G_{t}$ el gasto del gobierno, y $P_{t}$ el nivel de precios, se explica por dos componentes. Primero, por la producción doméstica $P_{m, t} Y_{t}-S_{t} M_{t}$ neta de las importaciones $M_{t}$ que se usan como insumos productivos, es decir, se debe restar al valor de la producción $\left(P_{m, t} Y_{t}\right)$ el valor de las importaciones $\left(S_{t} M_{t}\right)$, donde $S_{t}$ es el tipo de cambio nominal. Segundo, el gasto es también financiado a través del financiamiento externo o deuda externa neta de los pagos de intereses $S_{t} B_{t+1}^{*}-S_{t} R_{t}^{*} B_{t}^{*}$, donde $B_{t}^{*}$ es la deuda externa y $R_{t}^{*}$ la tasa de interés externa.

$$
\underbrace{P_{t} C_{t}+P_{t} I_{t}+P_{t} G_{t}}_{\text {gasto total }} \leq \underbrace{P_{m, t} Y_{t}-S_{t} M_{t}}_{\text {produccion neta }}+\underbrace{S_{t} B_{t+1}^{*}-S_{t} R_{t}^{*} B_{t}^{*}}_{\text {deuda externa neta }}+\underbrace{\left(S_{t} P_{t}^{c u} \overline{C U}\right)}_{\text {Elemento adicional }}
$$

De este modo, los modelos que incorporan la producción del commodity en forma exógena, adicionan un elemento extra en la restricción (ver ecuación 1.1), el cual corresponde al valor de las exportaciones de dicho commodity $\left(S_{t} P_{t}^{c u} \overline{C U}\right)$, donde es $P_{t}^{c u}$ el precio en dólares y $\overline{C U}$ es la cantidad fija que se exporta en cada período.

En la modelación descrita, toda la influencia del commodity en el modelo DSGE queda reducida a las fluctuaciones del precio $P_{t}^{c u}$. Al respecto, hay dos formas de modelar este precio, como un shock exógeno (ejemplo García y González 2014) o como un valor determinado endógenamente por las estructuras que representan al resto del mundo en el modelo (Laxton y Pesenti 2003). En este último caso, se debe modelar la economía internacional completa y no solo el país en cuestión, por esta razón en la literatura de los últimos años la primera alternativa es más popular que la segunda.

El Gráfico 2, reproducido de García et al (2011), ilustra los efectos de un shock positivo en el precio del cobre sobre las principales variables macroeconómicas en un modelo típico donde la producción del commodity es exógena. El ejercicio de simulación que muestra el mencionado gráfico se hace suponiendo dos escenarios alternativos para la política fiscal, puesto que en el modelo el 
gobierno es propietario de una parte importante de las empresas mineras: en un caso, que se denomina "presupuesto equilibrado", el gobierno se gasta todo el aumento del precio del cobre; en el otro, el gobierno decide usar una "regla fiscal", con lo cual se va gastando a través del tiempo el aumento del precio del cobre. Los autores demuestran que los efectos de un aumento en el precio del cobre sobre la economía son diferentes dependiendo de la política fiscal, en especial la respuesta de la inversión privada y el tipo de cambio real (en el gráfico se muestra el inverso de esta variable).

Si el gobierno usa una regla fiscal, como en el caso chileno, el aumento en la cantidad de dólares (el precio del cobre se expresa en dólares) no presionan a la baja el tipo de cambio porque los dólares se usan para el ahorro y no se gastan en el período. Por otro lado, los efectos inflacionarios son menores porque se privilegia el ahorro fiscal, con lo cual la tasa de interés (manejada por el banco central) no sube y por tanto la inversión se incrementa.

Por el contrario, en el caso de presupuesto equilibrado, el mayor gasto presiona al alza la inflación y con ello la tasa de interés. Como resultado final cae fuertemente tanto el tipo de cambio real como la inversión.

\section{Gráfico 2: Efecto de un aumento del precio del cobre en un modelo DSGE donde la producción de cobre es exógena.}
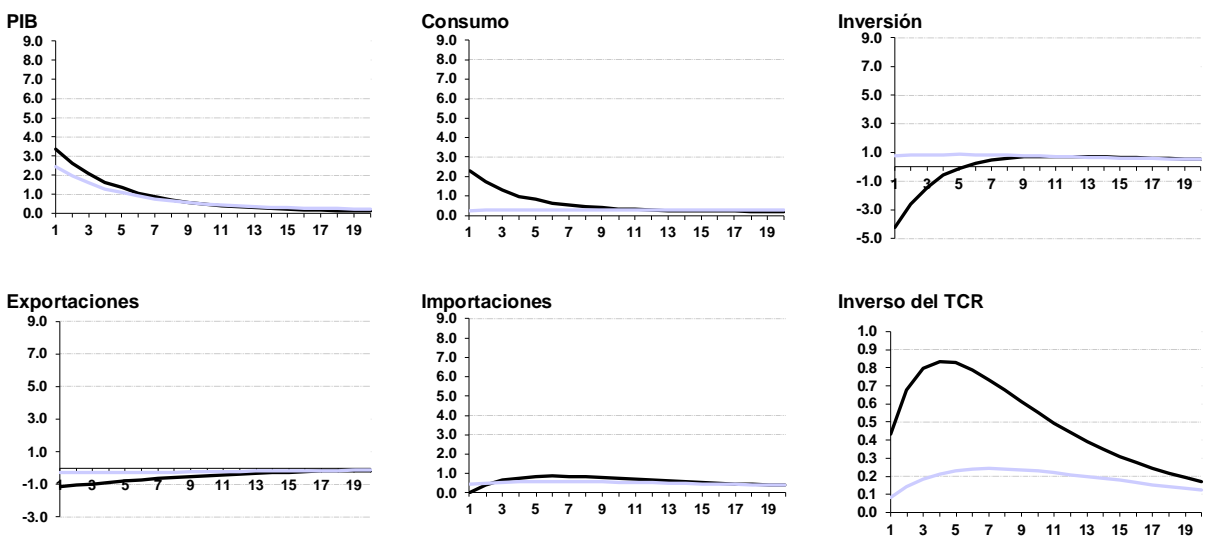

Fuente: García et al (2011)

Como alternativa a lo descrito, la literatura que endogeniza la producción de un commodity minero en un modelo DSGE es muy escasa. Dos artículos seminales en esta dirección son los de Gross y Hansen (2013) y Veroude (2012), quienes introducen en un modelo DSGE elementos de la 
vasta literatura de extracción óptima ${ }^{10}$. Ambos artículos parten por suponer una función de producción para la extracción de mineral $X$ que depende del trabajo $L$, el capital $K$ y un mineral $R$, tal como se expresa en la siguiente ecuación.

$$
X_{t}=L_{t}^{\alpha} K_{t}^{\beta} R_{t}^{1-\alpha-\beta}
$$

De este modo, las firmas mineras están sujetas a dos restricciones, por un lado, la formación de capital, y por otro, la reducción de las reservas de mineral. En términos muy simples se supone que el capital evoluciona de la manera estándar, aumentando por la inversión $I_{t}$ pero que se reduce de acuerdo a una tasa de depreciación $\delta$, lo cual se observa en la expresión:

$$
K_{t+1}=(1-\delta) K_{t}+I_{t}
$$

Cabe destacar que la innovación de ambos artículos es suponer que las reservas futuras $R_{t+1}$ son iguales a las pasadas, menos la cantidad que se extrae $X_{t}$ más una medida que representa nuevos descubrimiento de mineral $D_{t}$ :

$$
R_{t+1}=R_{t}-X_{t}+\omega_{t+1} D_{t}
$$

En esta última ecuación, el parámetro $\omega$ mide la incertidumbre asociada a encontrar nuevos descubrimientos de yacimiento de mineral. Por tanto, en virtud de la modelación la empresa representativa del sector minero enfrenta un trade -off entre extraer más mineral en el presente a costa de reducir sus reservas, en un contexto de incertidumbre respecto de encontrar nuevos yacimientos en el futuro.

En definitiva, la firma debe derivar sus costos marginales de (1.2) y maximizar beneficios sujetos a (1.3) y (1.4). Dada las características dinámicas y estocásticas de este problema, la solución se debe conseguir obteniendo las condiciones de primer orden de un problema de programación dinámica.

El Gráfico 3 muestra las diferencias entre un modelo con oferta exógena vs un modelo con oferta endógena. Frente a un aumento exógeno en el precio del cobre, los ingresos aumentan en A en el primer caso, en cambio en el segundo caso aumentan en $\mathrm{A}+\mathrm{B}+\mathrm{C}+\mathrm{D}$.

Teniendo presente lo antes expuesto, la conclusión preliminar a la que es razonable llegar parece clara: al suponer una oferta endógena en la producción de un commodity minero, los efectos

${ }^{10}$ Esta literatura trata de determinar hasta cuando es óptimo la extracción de un recursos no renovable (ver, por ejemplo, Bohn y Deacon 2000). 
sobre la economía de los cambios en algunas variables fundamentales serán más fuertes que los propuestos por modelaciones que no incluyen esta endogeneidad (por ejemplo, Garcia et al. (2011) ver Gráfico 2 -). No obstante lo indicado, no debiera esperarse una variación en la dirección de los efectos (los signos). De esta manera, se puede indicar que los trabajos tradicionales que suponen una oferta exógena han sistemáticamente sub-valuado los verdaderos efectos macroeconómicos del sector minero en la economía. La presente investigación intentará justamente medir estos efectos en un modelo dinámico con un sector minero endógeno.

\section{Gráfico 3: Oferta exógena vs endógena en modelos DSGE}

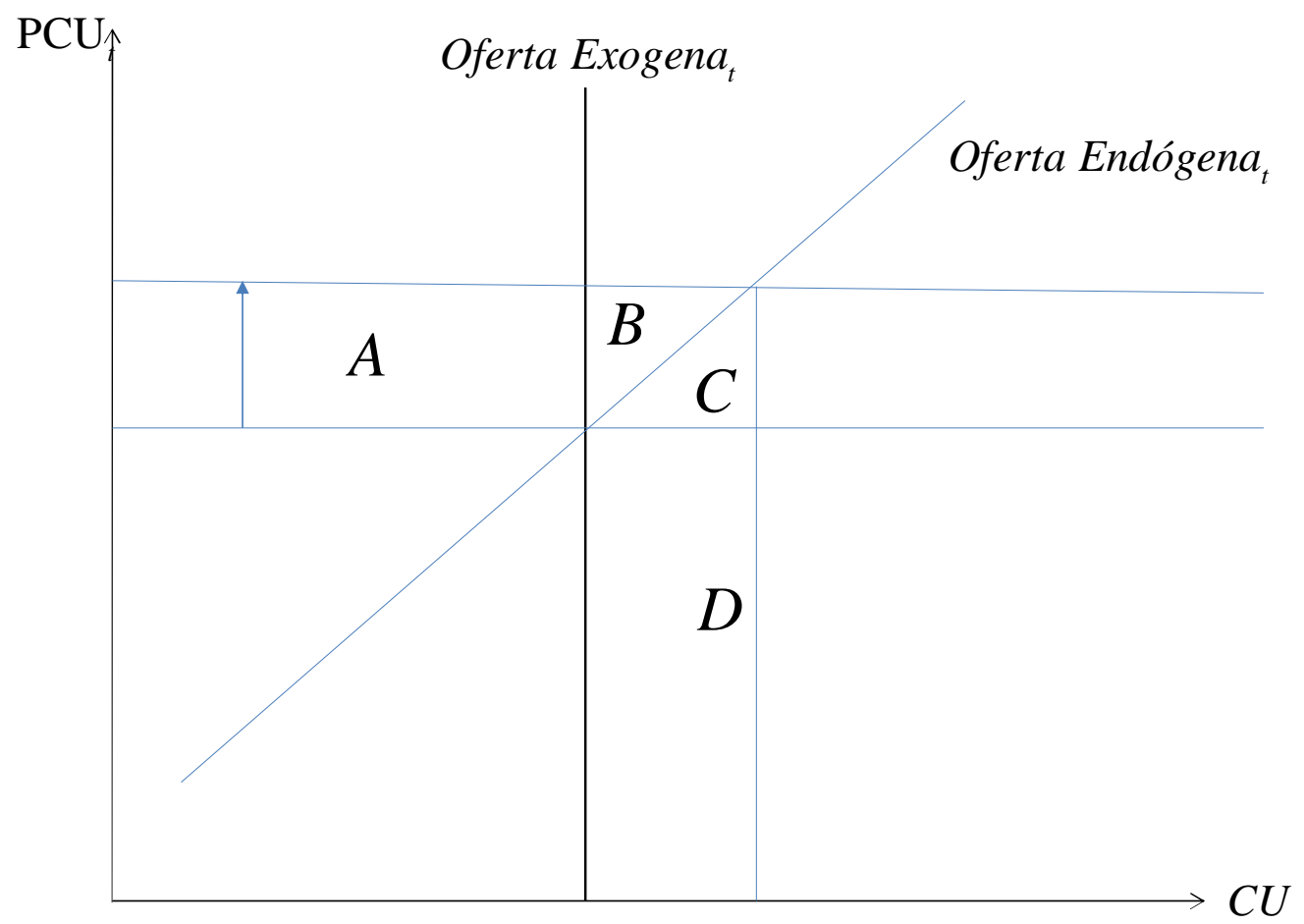




\section{SECTOR Minero Y CicLO ECONÓMICO CHILENO}

En este capítulo se presenta evidencia sobre los co-movimientos (correlaciones dinámicas) entre el sector minero y el ciclo económico de la economía chilena. La muestra utilizada, dependiendo de la disponibilidad de datos, fue idealmente de frecuencia trimestral y abarcó desde 2000-hasta 2013. Específicamente, se llevarán a cabo los siguientes análisis: (a) presentación gráfica de las relaciones entre las variables macroeconómicas elegidas y el sector minero, representado por el precio del cobre y el PIB sectorial; (b) correlación dinámica entre las variables macroeconómicas asociadas a las cuentas nacionales y el precio de cobre; (c) correlación dinámica entre las variables macroeconómicas asociadas a las cuentas nacionales y el PIB minero; (d) correlación dinámica entre los PIB sectoriales y el precio del cobre; (e) correlación dinámica entre el sector fiscal y el precio del cobre; (f) correlación dinámica entre variables del sector externo y el precio del cobre (g) correlación dinámica entre mercado laboral y el tipo de cambio real, respecto del precio del cobre; y (h) descripción sectorial cobre la importancia del sector minero.

Se ha optado por usar el tipo de análisis indicado de correlaciones, debido a que: permite visualizar la interacción entre el sector minero y el ciclo económico de Chile en la última década; entrega información adicional respecto a los trabajos antes citados en relación al impacto del sector minero en la economía; y, por sobre todo, representa una primera aproximación del análisis que se llevará a cabo en el contexto de la estimación del modelo DSGE en que la producción del sector minero se incorpora endógenamente.

Las correlaciones cruzadas entre la serie económica que representa el sector minero " $m$ " (precio del cobre y/ o PIB minero) y la serie macroeconómica " $y$ ” (PIB, consumo, tipo de cambio real, inversión, etc.) se miden de la siguiente forma:

$$
r_{m, y}(l)=\frac{C_{m, y}(l)}{\sqrt{C_{m, m}(0)} \sqrt{C_{y, y}(0)}} \quad l=0,+1,+2, \ldots
$$

Donde: 
$C_{m, y}(l)=\sum_{t=1}^{T-l}\left(\left(m_{i}-\bar{m}\right)\left(y_{i+l}-\bar{y}\right)\right) / T \quad l=0,1,2, \ldots$

$C_{m, y}(l)$ covarianza entre $m$ e $y$.

$C_{m, m}(0)$ varianza de $m$.

$C_{y, y}(0)$ varianza de $y$.

Lo que la ecuación 1.5 permite estimar es si las variables que representan al sector minero "adelantan" los movimientos de las series macroeconómicas, coinciden con dichos movimientos en forma contemporánea, o simplemente no se relacionan con ellos. De esta manera, se establece que la variable minera " $m$ " antecede el ciclo de una variable macroeconómica por " $T-l$ " períodos (trimestres 0 años), si dicha correlación es significativa (positiva o negativa) para un $T-l>0$ (García et al 2007).

La significancia estadística de una correlación específica, es decir, si esta correlación es diferente de cero, se mide analizando si el valor de la correlación está o no fuera de una banda de confianza de dos desviaciones estándares, representada por: $\pm 2 / \sqrt{N}$, donde $N$ es el número de observaciones.

Como primera aproximación, el Gráfico 4 muestra la relación contemporánea entre las diferentes variables de cuentas nacionales (CCNN) y las variables mineras (precio del cobre y PIB minero). Así, la diagonal del Gráfico 4, indica la relación de una misma variable medida tanto en el eje x como en el eje y, debiendo ser una línea de 45 grados. Fuera de la diagonal, el Gráfico 4 señala las relaciones contemporáneas entre las diferentes variables en dos ejes alternativos, dependiendo si se mira la diagonal triangular superior o inferior del gráfico (scatter plot). 


\section{Gráfico 4: Scatter Plot entre variables mineras y variables de CCNN}

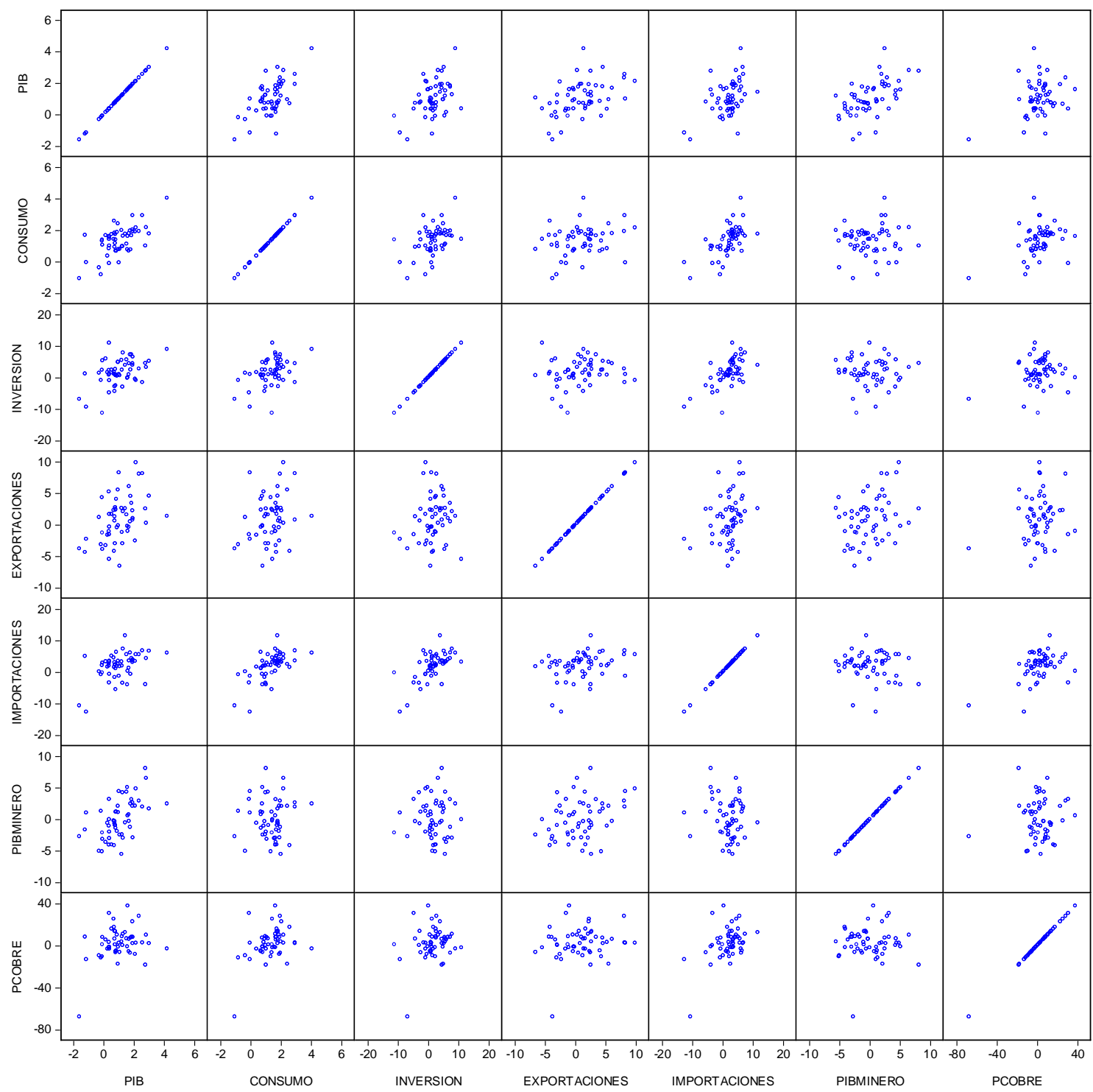

Fuente: elaboración de los autores.

En el Gráfico 4 se puede apreciar el primer resultado de esta sección y que se confirmará cuando se presenten las correlaciones dinámicas: se observa una relación positiva aunque débil entre el precio del cobre y la mayoría de las variables de CCNN, situación que ocurre en bastante menor medida con el PIB minero. Así, parece ser que el sector minero afectaría al resto de la economía a nivel macroeconómico a través del precio del cobre.

La Tabla y Figura 1 mide las correlaciones dinámicas entre el precio del cobre y las variables macroeconómicas de CCNN: consumo privado, inversión privada, exportaciones e importaciones, 
todo medido en términos reales. Todas las variables fueron expresadas como tasas de crecimiento trimestral ${ }^{11}$.

\section{Tabla y Figura 1: Correlaciones Dinámicas Sector Minero (precio del cobre) y CCNN}

Tabla: Correlaciones Dinámica entre precio del cobre y adelantos de las variables de CCNN

\begin{tabular}{c|ccccc}
\hline Adelantos & PIB & Inversión & Consumo & Importaciones & Exportaciones \\
\hline 0 & 0.2937 & 0.1904 & $0.3835^{*}$ & $0.4512^{*}$ & 0.1554 \\
1 & $0.3206^{*}$ & $0.4203^{*}$ & $0.3614^{*}$ & $0.5708^{*}$ & 0.1168 \\
2 & 0.2073 & 0.3018 & $0.344^{*}$ & $0.375^{*}$ & 0.0982 \\
3 & 0.0892 & 0.024 & 0.2043 & -0.0009 & 0.1235 \\
4 & 0.1085 & 0.067 & 0.1617 & -0.116 & -0.0797 \\
5 & 0.1622 & 0.0108 & 0.0893 & 0.0291 & 0.1754 \\
6 & -0.1683 & -0.0088 & -0.1586 & -0.0656 & -0.1762 \\
7 & -0.116 & 0.1954 & -0.0876 & 0.0458 & -0.0793 \\
8 & 0.0827 & 0.1532 & -0.0661 & 0.0885 & 0.0465 \\
9 & 0.0704 & -0.0134 & 0.0109 & -0.0995 & 0.0425 \\
10 & -0.0493 & -0.0072 & -0.1058 & -0.135 & -0.0886 \\
11 & -0.2165 & -0.2155 & -0.1847 & -0.2669 & -0.2138 \\
12 & -0.1457 & -0.1611 & -0.0875 & -0.0769 & -0.0872 \\
13 & -0.11 & -0.079 & -0.0837 & -0.0323 & 0.0885 \\
14 & -0.1072 & -0.0961 & -0.011 & -0.0107 & 0.0442 \\
15 & -0.0836 & -0.2022 & -0.0872 & -0.0378 & 0.0141 \\
16 & -0.0427 & 0.0193 & -0.0162 & -0.0462 & -0.1344 \\
* Significativo, mayor que dos errores estándars. & & & \\
Gráfico: Correlaciones Dinámica entre PIB minería y adelantos de las variables de CCNN
\end{tabular}

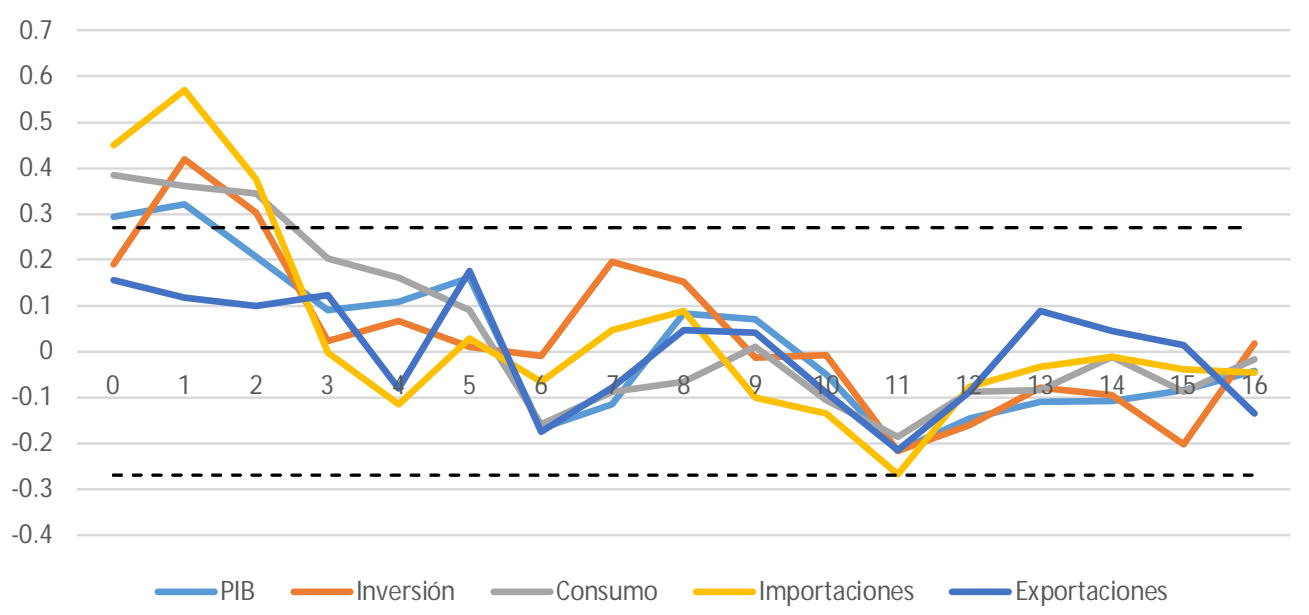

Banda de confianza de dos errores estándars se muestra con líneas discontinuas negras.

Fuente: elaboración de los autores.

Estas correlaciones indican que los crecimientos futuros del consumo, el PIB, la inversión y las importaciones co-varían positivamente con variaciones presentes en el precio del cobre. No

11 Una alternativa es usar el componente cíclico de la descomposición que hace el filtro de Hodrick Prescott (García et al 2007 y Restrepo y Soto 2006). 
obstante, es importante aclarar que el análisis de esta sección es sólo de co-movimiento entre variables y no de causalidad. Asimismo, es ilustrativo corroborar que el precio del cobre "adelanta" en forma débil12 pero positivamente a variables como la inversión.

Por el contario, las exportaciones totales no muestran ningún tipo de co-movimiento futuro con el precio del cobre. Es relevante este punto porque el co-movimiento tampoco es negativo, lo cual sería un indicador, por ejemplo, de síntomas de la enfermedad holandesa que se le atribuye a los booms en el precio de los commodities, es decir, el fenómeno asociado a que un precio muy alto en el cobre podría estar apreciando el tipo de cambio real y con ello causando pérdida de competitividad al sector industrial.

La Tabla y Figura 2 muestran las mismas correlaciones, pero usando como variable representativa del sector el PIB minero, medido en términos reales y como tasas de crecimiento trimestrales. Los resultados son menos definitivos que los anteriores, indicando que existe una correlación contemporánea con el PIB total y las exportaciones totales. Es probable que el comovimiento capture el simple hecho que el PIB minero es un porcentaje importante del PIB y de las exportaciones totales. Por otra parte, el Gráfico 5 indica que el sector minero ha tendido a decrecer como porcentaje del PIB en relación a otras actividades económicas. Resultado que es coherente con el hecho que si bien la correlación entre el PIB y el PIB minero es positiva, es débil.

\section{Gráfico 5: Participaciones de sectores seleccionados en el PIB total}

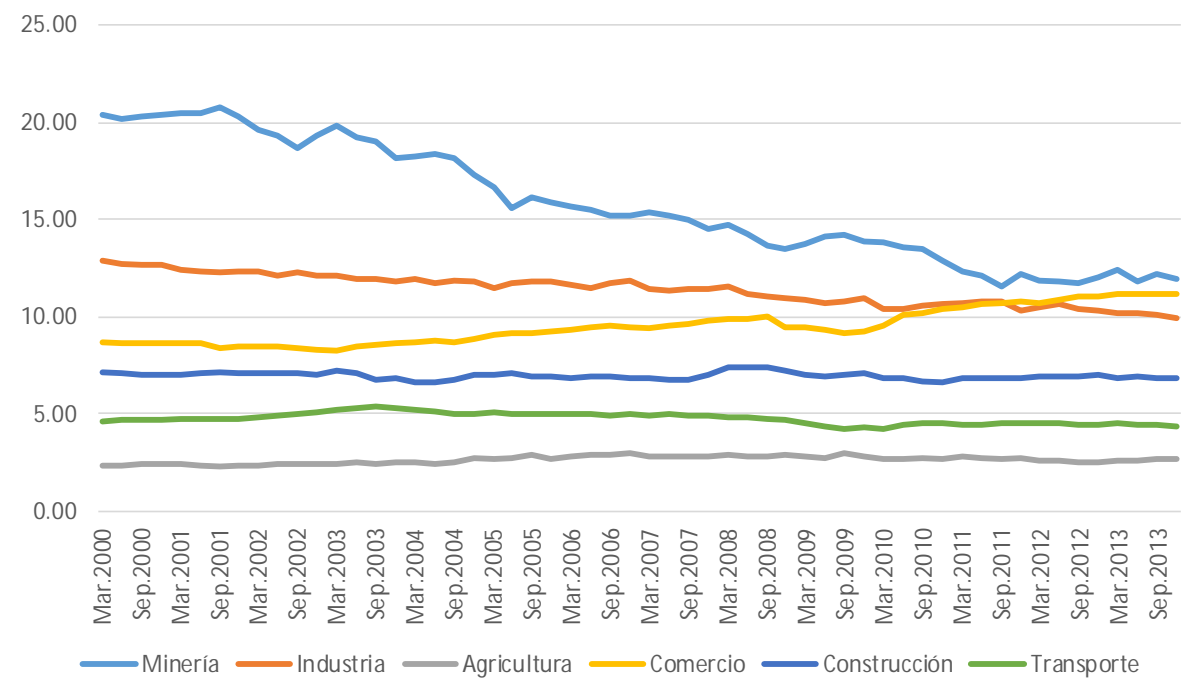

Fuente: Banco Central de Chile y autores.

12 En adelante, "débil" debe entenderse como una correlación estadísticamente significativa, pero muy cercana a la banda de confianza. 


\section{Tabla y Figura 2: Correlaciones Dinámicas Sector Minero (PIB minero) y CCNN}

Tabla: Correlaciones Dinámica entre PIB minero y adelantos de las variables de CCNN

\begin{tabular}{c|ccccc}
\hline Adelantos & PIB & Inversión & Consumo & Importaciones & Exportaciones \\
\hline 0 & $0.5532^{*}$ & -0.011 & 0.0506 & -0.1054 & $0.3155^{*}$ \\
1 & -0.054 & -0.0226 & 0.2295 & 0.2214 & -0.0169 \\
2 & 0.0537 & 0.0889 & -0.0176 & 0.1893 & 0.0397 \\
3 & -0.1639 & 0.0858 & 0.0158 & -0.0275 & -0.2243 \\
4 & -0.0046 & -0.098 & 0.0994 & 0.1118 & 0.2333 \\
5 & 0.156 & -0.0693 & 0.0261 & -0.1531 & -0.0853 \\
6 & -0.0077 & 0.0932 & 0.0423 & 0.0135 & 0.1766 \\
7 & -0.0845 & -0.0851 & -0.3014 & -0.0798 & -0.0091 \\
8 & -0.3543 & -0.1877 & -0.2889 & -0.1805 & -0.3806 \\
9 & 0.0237 & 0.1767 & -0.0856 & -0.0552 & 0.0273 \\
10 & 0.1605 & -0.0632 & 0.0173 & -0.0318 & 0.1818 \\
11 & -0.0584 & -0.0331 & 0.0394 & -0.0285 & -0.1229 \\
12 & -0.1001 & 0.0245 & -0.0772 & 0.0628 & 0.1352 \\
13 & -0.0304 & -0.1807 & -0.0182 & -0.03 & -0.1136 \\
14 & 0.1128 & -0.011 & 0.1809 & 0.0556 & 0.1103 \\
15 & 0.2128 & 0.1393 & 0.1485 & 0.1632 & 0.1083 \\
16 & 0.1556 & 0.1767 & 0.1896 & 0.1541 & 0.0717
\end{tabular}

Gráfico: Correlaciones Dinámica entre PIB minero y adelantos de las variables de CCNN

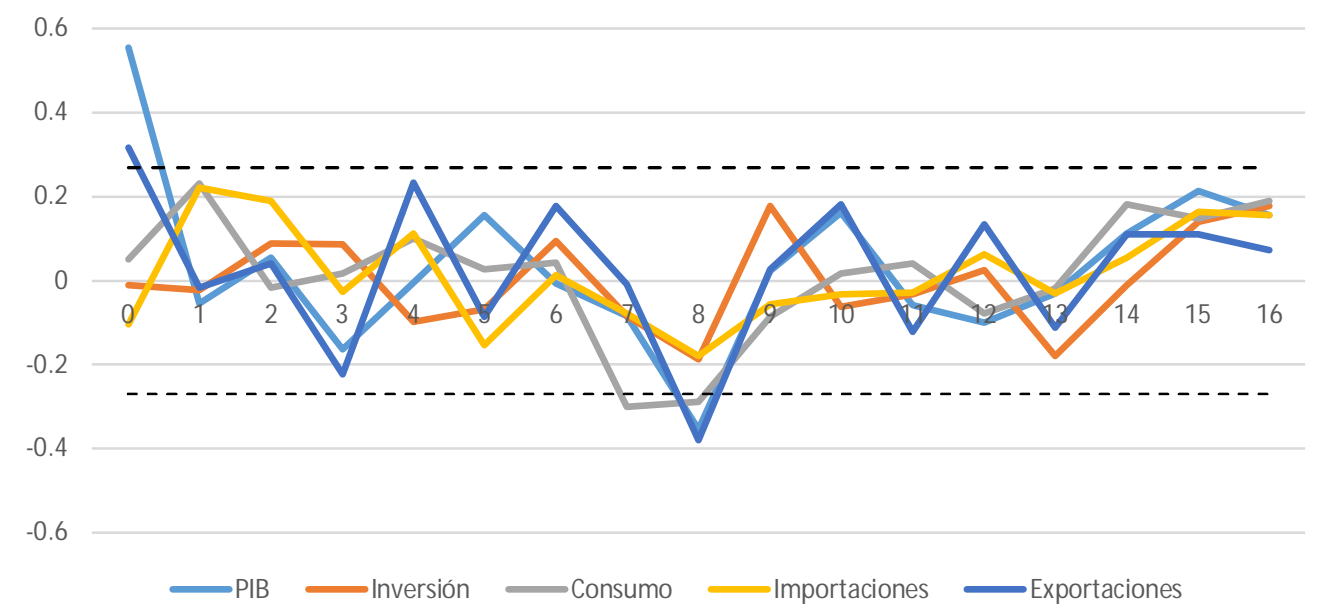

Explicaciones ver Tabla y Figura 1.

Fuente: elaboración de los autores.

Dado que el PIB minero no muestra una correlación muy relevante respecto a las variables macroeconómicas analizadas, lo que sigue de este capítulo se focalizará en el precio del cobre como variables sectorial fundamental.

La Tabla y Figura 3 muestran los resultados de las correlaciones dinámicas entre el precio del cobre y los PIB sectoriales. Este precio co-varía positivamente con el sector comercial, en primer lugar, y luego con el sector industrial. Aunque, es importante hacer notar que la correlación con el sector industrial y el comercial no necesariamente indica que el sector minero pueda afectar 
positivamente a estos sectores en términos de causalidad. En efecto, podría ser también que exista una tercera variable o fuerza económica (por ejemplo el ciclo económico internacional) que esté afectando a todas las variables en la misma dirección. Por último, no hay evidencia que el precio del cobre co-varíe ni con la construcción ni con el sector agrícola.

\section{Tabla y Figura 3: Correlaciones Dinámicas Sector Minero (Precio del Cobre) y PIB sectoriales}

Tabla: Correlaciones Dinámica entre Precio del Cobre y adelantos del PIB de sectores

\begin{tabular}{c|ccccc}
\hline Adelantos & Agricola & Comercial & Industria & Cosntrucción & Transporte \\
\hline 0 & 0.0541 & $0.4254^{*}$ & $0.3835^{*}$ & 0.2145 & 0.1253 \\
1 & 0.2793 & $0.3213^{*}$ & $0.3614^{*}$ & 0.2398 & 0.2316 \\
2 & 0.0023 & $0.2837^{*}$ & $0.344^{*}$ & 0.1318 & $0.3091^{*}$ \\
3 & -0.1391 & 0.2115 & 0.2043 & 0.0443 & 0.0914 \\
4 & 0.0821 & 0.1765 & 0.1617 & -0.0559 & 0.1263 \\
5 & 0.2889 & 0.016 & 0.0893 & 0.1723 & 0.045 \\
6 & 0.0356 & $-0.3144^{*}$ & -0.1586 & -0.0085 & $-0.3754^{*}$ \\
7 & -0.1641 & 0.01 & -0.0876 & 0.2086 & -0.2536 \\
8 & -0.0475 & 0.0411 & -0.0661 & 0.1244 & 0.0651 \\
9 & -0.0109 & 0.0358 & 0.0109 & 0.0414 & 0.1082 \\
10 & 0.0533 & -0.1916 & -0.1058 & 0.0219 & 0.0007 \\
11 & -0.1664 & -0.1448 & -0.1847 & -0.1833 & $-0.2772 *$ \\
12 & -0.0656 & -0.1104 & -0.0875 & -0.0954 & -0.101 \\
13 & 0.132 & -0.1883 & -0.0837 & -0.1429 & -0.1712 \\
14 & -0.097 & -0.159 & -0.011 & -0.0557 & -0.1168 \\
15 & -0.0181 & -0.0456 & -0.0872 & -0.0481 & -0.0861 \\
16 & -0.0533 & 0.0603 & -0.0162 & 0.0004 & 0.0053
\end{tabular}

Gráfico: Correlaciones Dinámica entre Precio del Cobre y adelantos del PIB de sectores

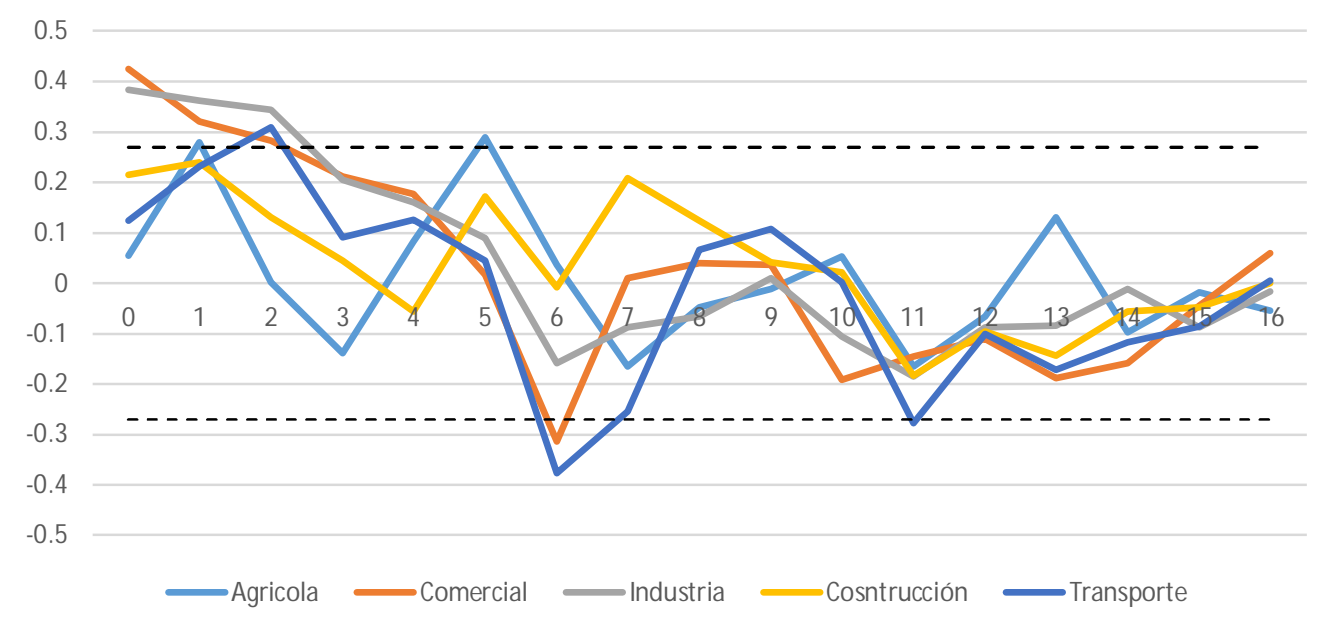

Nota: explicaciones ver Tabla y Figura 1.

Fuente: elaboración de los autores. 
La Tabla y Figura 4 muestran, para datos anuales, las correlaciones dinámicas entre el precio del cobre y los gastos e ingresos fiscales, expresados como porcentajes del PIB. Como era de esperarse, existe un co-movimiento contemporáneo entre el precio del cobre y los ingresos fiscales provenientes del cobre. Sin embargo, un resultado que resalta es que cambios en el precio del cobre adelantan aumentos en los diferentes gastos del fisco como porcentaje del PIB. Este resultado es coherente con una regla fiscal que indica ir gastando los ingresos del cobre a través del tiempo (García et al. 2011) y no inmediatamente.

\section{Tabla y Figura 4: Correlaciones Dinámicas Sector Minero (precio del cobre) y sector fiscal (gastos e ingresos)}

Tabla: Correlaciones Dinámica entre Precio del Cobre y adelantos de las siguientes variables como porcentaje del PIB

\begin{tabular}{c|ccccc}
\hline Adelantos & Gasto Personas & Transferencias & Gasto Total & Ing Tributarios & Ing Cobre \\
\hline 0 & -0.2766 & 0.4325 & -0.3055 & 0.5138 & $0.5714^{*}$ \\
1 & 0.0615 & $0.5707^{*}$ & 0.0303 & 0.4515 & 0.2084 \\
2 & 0.3703 & $0.681^{*}$ & 0.3645 & 0.1567 & -0.0858 \\
3 & $0.5482^{*}$ & $0.6368^{*}$ & 0.5444 & -0.0019 & -0.3263 \\
4 & $0.5762^{*}$ & 0.5032 & 0.5389 & 0.1354 & -0.4802 \\
5 & 0.4447 & 0.2405 & 0.4037 & 0.0663 & -0.4854 \\
6 & 0.258 & -0.0438 & 0.2174 & 0.0221 & -0.3859
\end{tabular}

Gráfico: Correlaciones Dinámica entre Precio del Cobre y adelantos de las siguientes variables como porcentaje del PIB

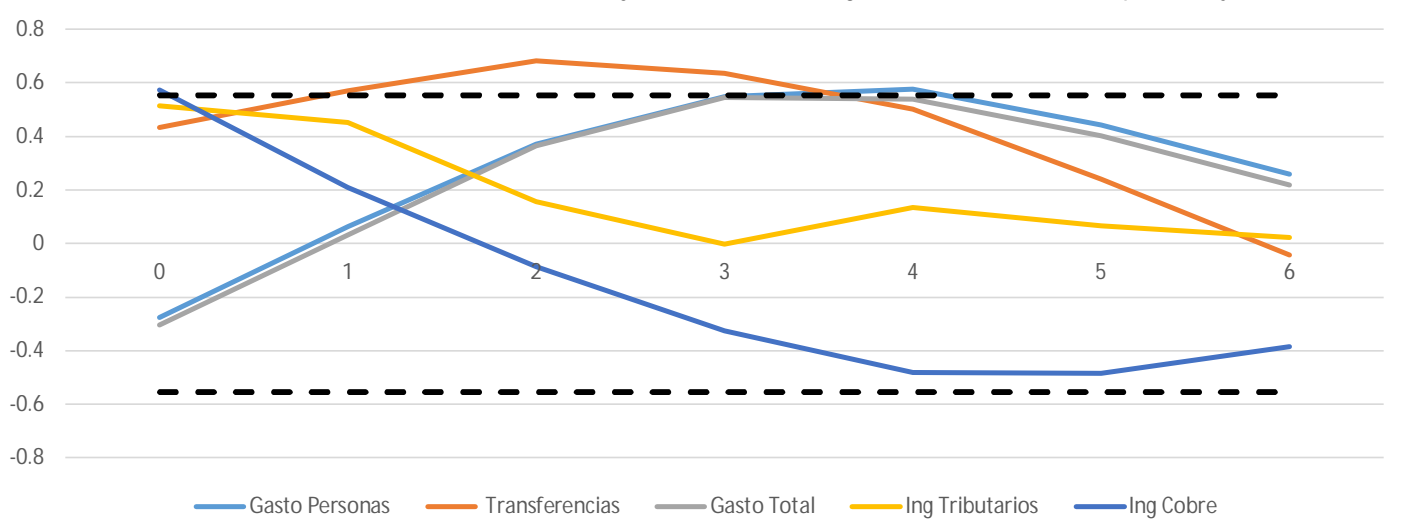

Explicaciones ver Tabla y Figura 1.

Fuente: elaboración de los autores.

La Tabla y Figura 5 muestran, para datos trimestrales, las correlaciones dinámicas entre el precio del cobre y variables del sector externo medidos en como porcentajes del PIB (cuenta corriente e inversiones extranjeras), junto a tasas de crecimientos dólares de las exportaciones. Todas las variables fueron medidas en dólares de cada año, incluso la cuenta corriente y la inversión extranjeras antes de ser divididas por el PIB en dólares. Los resultados indican que el precio del cobre adelanta por muchos períodos la inversión extranjera sobre el PIB. Además, coincide contemporáneamente con un superávit de cuenta corriente. Ambos resultados son coherentes con 
el hecho de que los precios más altos del cobre de los últimos años han atraído inversiones extranjeras, y con la evidencia de que un buen precio permite alanzar un superávit de cuenta corriente a través de su efecto en la balanza comercial.

\section{Tabla y Figura 5: Correlaciones Dinámicas Sector Minero (precio del cobre) y sector externos.}

Tabla: Correlaciones Dinámica entre Precio del Cobre y adelantos las siguientes variables

\begin{tabular}{c|ccccc}
\hline Adelantos & Cta Corriente & Inv Extranjera & Exp Industria & Exp. Agricola & Exp. Mineras \\
\hline 0 & $0.5107^{*}$ & $0.5518^{*}$ & $0.677^{*}$ & $0.4815^{*}$ & $0.6292^{*}$ \\
1 & $0.3196^{*}$ & $0.5276^{*}$ & $0.3476^{*}$ & 0.0394 & 0.0052 \\
2 & 0.0751 & $0.5064^{*}$ & 0.0146 & 0.0709 & -0.1842 \\
3 & -0.1319 & $0.4571^{*}$ & -0.1035 & -0.0123 & -0.0632 \\
4 & -0.2508 & $0.4869^{*}$ & 0.078 & 0.1017 & -0.1164 \\
5 & -0.2913 & $0.5075^{*}$ & 0.2427 & -0.1207 & 0.161 \\
6 & $-0.3237^{*}$ & $0.4959^{*}$ & -0.0977 & -0.2111 & -0.0245 \\
7 & $-0.3583^{*}$ & $0.5136^{*}$ & 0.0575 & 0.2176 & 0.0813 \\
8 & $-0.3626^{*}$ & $0.468^{*}$ & 0.1426 & 0.1343 & -0.0081 \\
9 & $-0.3392^{*}$ & $0.4197^{*}$ & -0.0059 & 0.0318 & -0.0931 \\
10 & -0.2236 & $0.448^{*}$ & -0.411 & -0.3212 & -0.2498 \\
11 & -0.0595 & $0.4058^{*}$ & -0.2698 & -0.0558 & -0.1683 \\
12 & 0.1303 & $0.353^{*}$ & 0.0791 & -0.0697 & 0.037 \\
13 & 0.2782 & 0.2565 & 0.0412 & 0.1024 & 0.1962 \\
14 & $0.3507^{*}$ & 0.1469 & -0.0011 & 0.1173 & 0.1062 \\
15 & $0.3526^{*}$ & 0.0988 & -0.0018 & -0.0484 & -0.1463 \\
16 & $0.3228^{*}$ & 0.0646 & -0.0298 & 0.0678 & -0.0459
\end{tabular}

Gráfico: Correlaciones Dinámica entre Precio del Cobre y adelantos las siguientes variables como porcentaje del PIB

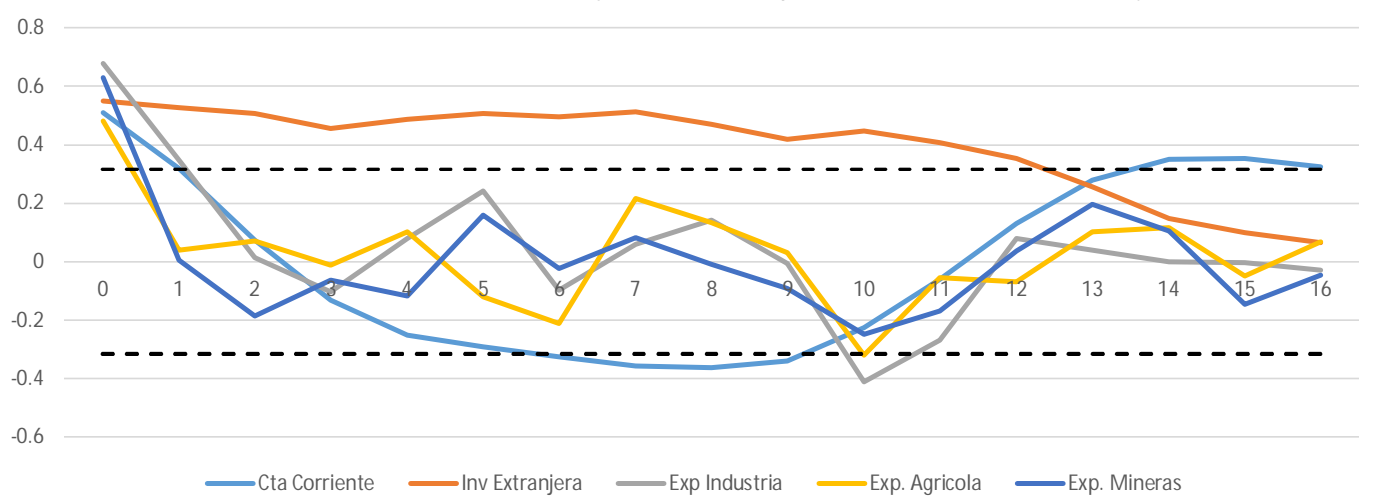

Notas: Cuenta corriente e Inversión extranjera se expresan como porcentajes del PIB, las exportaciones como tasas crecimiento trimestral.

El precio del Cobre fue expresado de diferentes formas. En la correlación con la inversión extranjera se usó la tendencia del filtro HP, en la cuenta corriente se usó la parte ciclica del filtro HP, y en las exportaciones se usó la tasa de crecimiento trimestral. (HP=Hodrick Prescott)

Explicaciones ver Tabla y Figura 1. Fuente: elaboración de los autores.

Por otra parte, el co-movimiento positivo entre el precio del cobre y el crecimiento de las exportaciones agrícolas e industriales en dólares puede estar indicando, como se mencionó anteriormente, que existe una tercera variable o fuerza económica que ha impulsado (o contraído) 
no sólo el precio del cobre sino también todo tipo de exportaciones. En este contexto, parece razonable suponer que las fluctuaciones de la economía internacional pudieran ser esta tercera variable o fuerza, representada primero el largo ciclo de crecimiento que se registró hasta el 2007,y después la fuerte contracción provocada por la crisis financiera internacional que comenzó el 2008 (y su posterior) recuperación.

La Tabla y Figura 6, muestra los co-movimientos entre el precio del cobre y variables relacionadas con el mercado laboral y el tipo de cambio real (TCR). Los resultados son claros en indicar que las correlaciones entre las variables del mercado laboral y le precio del cobre son estadísticamente cero o contra intuitivas (la correlación con los salarios reales es cero o negativa). Esta evidencia está en línea con la importancia del empleo del sector minero en el empleo total. El Gráfico 6 muestra las participaciones de diferentes sectores en el empleo a nivel nacional, relevando que el empleo en el sector minero es marginal, en torno al 3\%, magnitud muy pequeña en comparación a los principales sectores no mineros de la economía (industria, comercio y construcción), que suman casi el 55\% del empleo total de la economía Chilena. Por tanto, es muy probable que el sector minero no tenga efectos directos sobre el empleo, la tasa de desempleo y la evolución de los salarios reales a nivel nacional.

\section{Gráfico 6: Participaciones de sectores seleccionados en el empleo total}

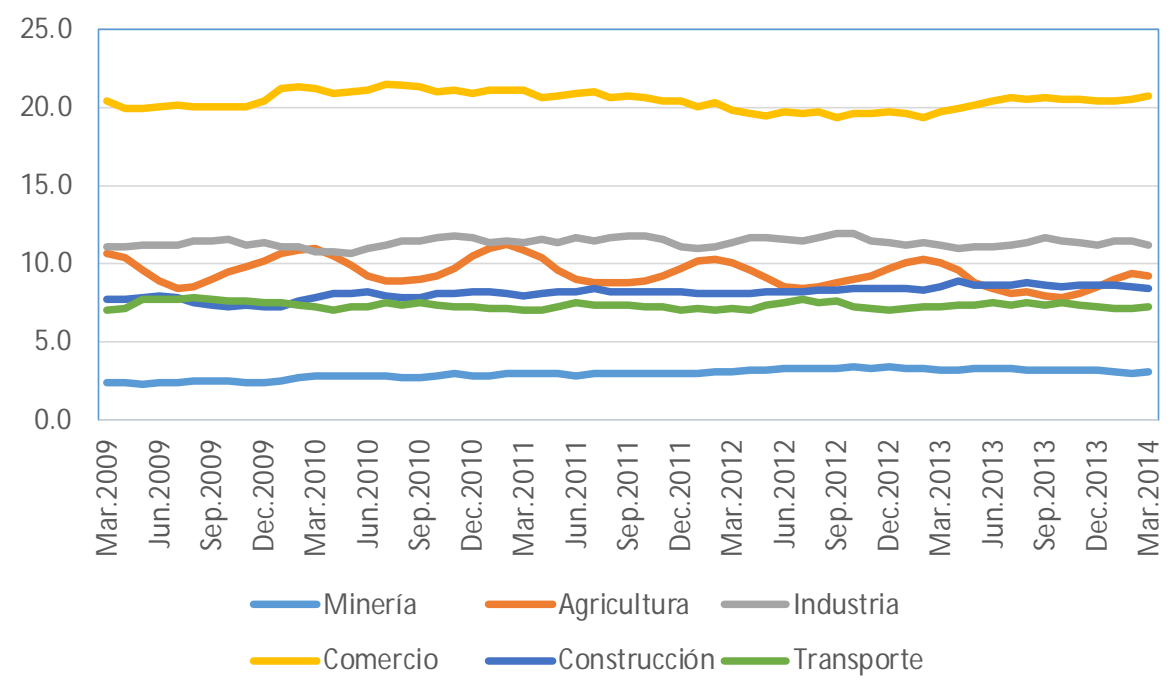

Fuente: Banco Central de Chile y autores.

Adicionalmente, y como era de esperar, la Tabla y Figura 6 indican que existe una relación negativa entre precio del cobre y el TCR. Aunque este co-movimiento es muy débil y sólo 
contemporánea. Esto resultado es consistente con evidencia sistemática a nivel nacional e internacional que muestra que el precio de los commodities no predice las fluctuaciones del tipo de cambio (Meese y Rogoff 1983, Chen et al 2010, García et al 2013).

\section{Tabla y Figura 6: Correlaciones Dinámicas Sector Minero (precio del cobre), mercado laboral y tipo de cambio real.}

Tabla: Correlaciones Dinámica entre Precio del Cobre y adelantos del crecimiento trimestral de las siguientes variables

\begin{tabular}{c|ccccc}
\hline Adelantos & Tasa de Desempleo & Empleo & Costo Mano de Obra Real & Remuneraciones Reales & TCR \\
\hline 0 & 0.1684 & 0.0221 & 0.0399 & 0.0685 & -0.408 \\
1 & 0.2077 & 0.1766 & $-0.3467 *$ & $-0.3344^{*}$ & 0.2214 \\
2 & 0.1408 & 0.1346 & -0.0801 & -0.0833 & 0.1231 \\
3 & -0.0604 & 0.1187 & 0.0587 & 0.0758 & 0.0125 \\
4 & -0.1576 & 0.1195 & 0.0411 & 0.0647 & 0.0676 \\
5 & -0.1301 & 0.019 & -0.1298 & -0.1106 & 0.0051 \\
6 & -0.1019 & $-0.3123 *$ & -0.0136 & 0.0188 & -0.1419 \\
7 & -0.1259 & -0.1416 & 0.0925 & 0.1247 & -0.2438 \\
8 & -0.0846 & 0.213 & -0.0766 & -0.0586 & 0.0775 \\
9 & -0.0851 & 0.0155 & -0.1327 & -0.1324 & 0.1643 \\
10 & -0.077 & -0.2929 & -0.0592 & -0.023 & 0.1641 \\
11 & -0.1502 & -0.0561 & 0.074 & 0.099 & -0.1046 \\
12 & -0.1709 & 0.2484 & 0.1139 & 0.1089 & -0.0615 \\
13 & -0.0767 & -0.054 & -0.0062 & 0.0084 & 0.0022 \\
14 & -0.0058 & $-0.4331^{*}$ & -0.0286 & 0.0174 & -0.0297 \\
15 & -0.0102 & 0.0365 & -0.0569 & -0.0409 & 0.1145 \\
16 & 0.0101 & $0.5146 *$ & 0.008 & -0.0157 & 0.0151
\end{tabular}

Gráfico: Correlaciones Dinámica entre Precio del Cobre y adelantos de las siguientes variables

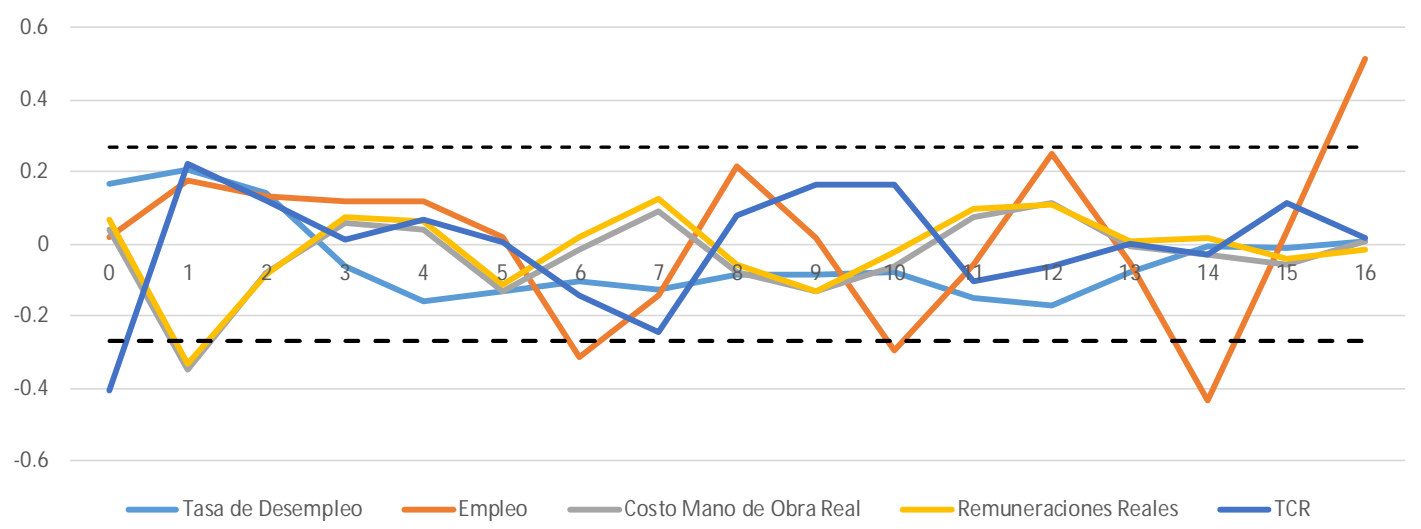

Explicaciones ver Tabla y Figura 1.

Fuente: elaboración de los autores.

Desde una perspectiva analítica diferente, para terminar este capítulo se ha tratado de medir la importancia del impacto regional del sector minero. Lamentablemente, la información es parcial y solo se cuenta con datos anuales. Así, según los antecedentes disponibles que tiene el Banco Central de Chile, se ha definido como regiones mineras a aquellas en donde el PIB sectorial es por lo menos un $40 \%$ del PIB regional total. Entonces, las regiones mineras abarcan desde la primera a la cuarta. 
Según se observa en la Tabla 7, la región más importante en términos agregados es la segunda región: produce el 10\% del PIB total, donde el 63\% corresponde al PIB minero de la región. Si bien no se tienen datos para hacer correlaciones dinámicas a nivel regional, es obvio que tanto el precio del cobre como la producción minera deben tener importantes efectos en estas regiones.

\section{Tabla 7: Participación de las Regiones en el PIB total.}

\begin{tabular}{c|ccccccc} 
& I De Tarapacá & || De Antofagasta & I| De Atacama & IV De Coquimbo & Regiones Mineras & RM & Resto Regiones no Mineras \\
2010 & $3 \%$ & $10 \%$ & $2 \%$ & $3 \%$ & $19 \%$ & $44 \%$ & $38 \%$ \\
2011 & $3 \%$ & $9 \%$ & $2 \%$ & $3 \%$ & $17 \%$ & $44 \%$ & $39 \%$ \\
2012 & $2 \%$ & $10 \%$ & $2 \%$ & $3 \%$ & $17 \%$ & $44 \%$ & $38 \%$
\end{tabular}

Regiones Mineras: I+II+III+IV, PIB minero es sobre el 40\% del PIB total de la región

Fuente: Banco Central de Chile y autores

Por otro lado, si bien el sector minero no tiene un impacto directo sobre el empleo a nivel nacional, la realidad regional es diferente. Si tomamos como ejemplo, la Segunda Región, ver Gráfico 7, la principal fuente de empleo es la minería seguido por el comercio. Nuevamente, si bien no se tienen datos para hacer un análisis de correlaciones dinámicas entre el empleo regional, el PIB minero y el precio del cobre, parece natural que estas últimas variables sean determinantes en la evolución del mercado laboral regional.

\section{Gráfico 7: Participaciones de sectores seleccionados en el empleo total de la Segunda Región}

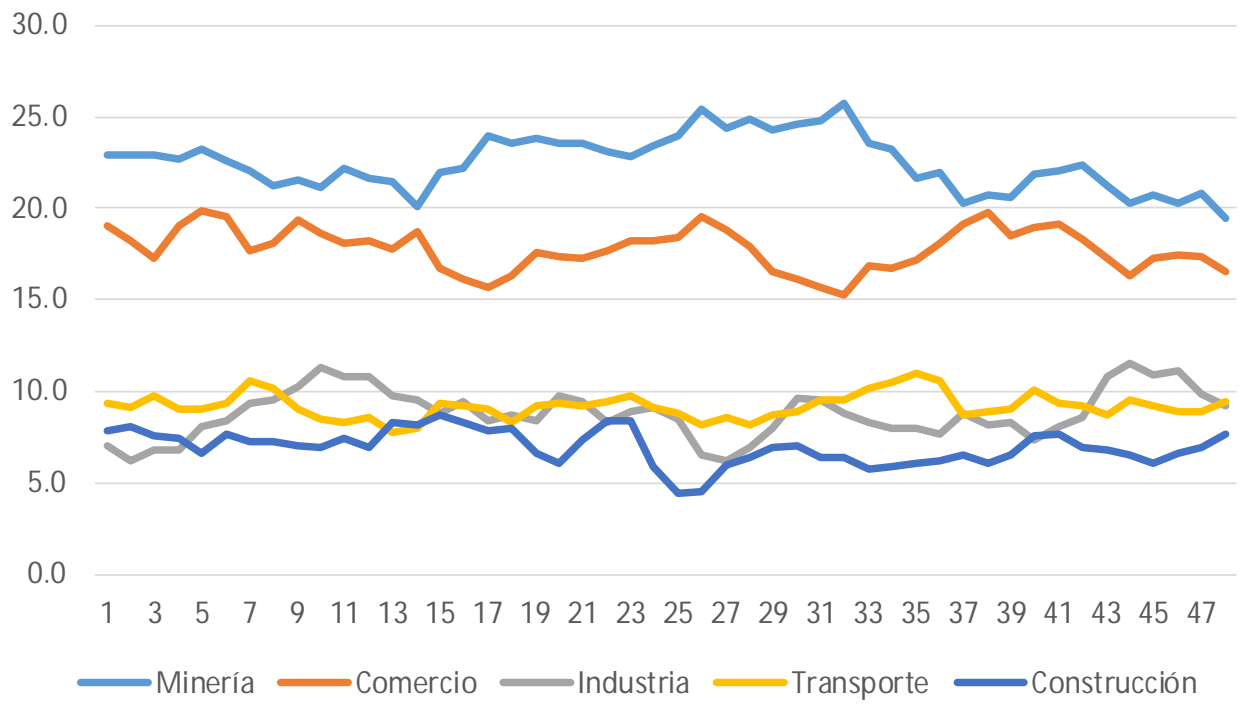

Fuente: INE y autores 
Como conclusión, cabe destacar que los hechos estilizados de la presente sección indican una serie de elementos que deben ser considerados al momento de modelar el sector minero en un modelo macro DSGE:

- El precio del cobre parece ser el elemento clave en resumir los efectos del sector minero en el resto de la economía.

- En la muestra que se consideró, el precio del cobre se adelanta a cambios en el PIB, la inversión privada, el consumo y las importaciones.

- El precio también se adelanta a aumentos en el gasto fiscal, aunque esto ocurre tres años después del aumento del precio del cobre.

- El precio del cobre también se adelanta a la inversión extranjera.

- El sector minero no tiene importantes efectos en el mercado laboral a nivel nacional. En este sentido, este sector funcionaría como un enclave con su propia fuerza laboral concentrada en las regiones primera, segunda, tercera y cuarta.

- Los efectos sobre el tipo de cambio real son negativos, pero no hay evidencia que los cambios del precio del cobre adelanten cambios en el tipo de cambio real.

- Si bien una caída del tipo de cambio real podría reducir la competitividad del sector exportador no minero, la evidencia encontrada en este estudio no muestra que la actividad minera explique caídas relevantes en el tipo de cambio. 


\section{MODELO MACROECONÓMICO Y ROL DEL SECTOR MINERO}

En esta sección se presentan las modificaciones claves realizadas en el modelo estándar DSGE (ver García y González 2014) que se debieron hacer para poder introducir apropiadamente el sector minero ${ }^{13}$. Las modificaciones fueron de dos tipos: por un lado, se modeló endógenamente la producción del sector minero; y por otro, como resultado de este cambio, se adicionó un nuevo canal de integración entre este sector y el resto de la economía -- la demanda por insumos intermedios --. De esta manera, se puede constatar que dado los supuestos del modelo empleado en el estudio, los insumos importados se transforman en bienes intermedios que satisfacen las demandas de consumo, la inversión del propio sector intermedio y del gobierno, y la inversión del sector minero. Por el contrario, el modelo estándar sólo enfatiza el aporte fiscal directo del sector minero, descontando las remesas de utilidades de las empresas mineras de origen externo.

En virtud de lo señalado, las modificaciones al modelo DSGE estándar son las dos que se exponen a continuación:

\section{i.) Sector Minero}

A diferencia del modelo DSGE estándar, se supuso que la producción de cobre no es exógena. Por el contrario, se asume que la producción de cobre $Q C U_{t}$ depende del trabajo $L_{t}^{C U}$, el capital $K_{t}^{C U}$ y la energía $E_{t}$.

$$
Q C U_{t}=A_{t}^{C U} L_{t}^{C U \alpha} K_{t}^{C U \beta} E_{t}^{1-\alpha-\beta}
$$

Donde $A_{t}$ representa la disponibilidad del mineral. En términos logarítmicos se supone que esta variable sigue la siguiente forma: $a_{t}=\left(r h o_{-} A_{-} C O B R E\right) a_{t-1}+\varepsilon_{t}^{E E}$.

\footnotetext{
${ }^{13}$ En el Anexo $\mathrm{N}^{\circ} 1$ se presenta el detalle de las ecuaciones del modelo empleado.
} 
La incorporación de estos tres insumos ( $L_{t}^{C U}, K_{t}^{C U}$ y $E_{t}^{C U}$ ) complejiza la modelación del modelo DSGE en varias dimensiones. Por lo tanto, se deben realizar una serie de supuestos para poder simplificar la mencionada modelación.

Primero, se supuso que el sector minero usa un compuesto de energía, formado por combustible (petróleo) y energía eléctrica. Para ambos insumos, se considera que el sector minero es un tomador de precio.

$$
E_{t}=O I L_{t}^{\delta} E E_{t}^{1-\delta}
$$

Donde, $O I L_{t}$ es el combustible y $E E_{t}$ es la energía eléctrica. De este modo, dado un cierto nivel de producción, y por tanto de energía total $\left(E_{t}\right)$, se pueden obtener por separado las demandas de combustible y de energía eléctrica en función de los respectivos precios. En términos logarítmicos se asume que el precio de la energía eléctrica sigue la siguiente forma: $p_{t}^{E E}=\left(r h o_{-} P E E\right) p_{t-1}^{E E}+\varepsilon_{t}^{E E}$.

No obstante lo indicado, para mejorar el ajuste del modelo DSGE se supuso además que existen rezagos en las respuestas no solo de la demanda de energía sino de todos los insumos a los respectivos precios. De esta manera, la demanda por un insumo genérico $J$ que se denomina insumo $_{J, t}$, "log linealizada", además de depender positivamente del nivel de producción producción $_{t}$ y negativamente del precio del insumo, expresado en términos reales, el cual se denomina $p_{J, t}$, también depende de un rezago en la demanda por el: insumo $_{J, t-1}$ :

$$
\begin{aligned}
\text { insumo }_{J, t} & =\text { pmg_insumo }_{J} \text { COBRE }\left(\text { producción }_{t}-p_{J, t}\right) \\
& +\left(1-\text { pmg_insumo }_{J} \text { COBRE }\right) \text { insumo }_{J, t-1}
\end{aligned}
$$

Donde $p m g_{-}$insumo $_{J_{-}} C O B R E$, representa un parámetro de la ecuación, el cual asume un valor entre cero y uno.

Segundo, las firmas productoras de cobre compran capital a otras firmas en cada período " $t$ ". Si bien en la realidad puede ser que algunas firmas produzcan también algunos de sus bienes de capital, se asume en este estudio, y solo por simplicidad, que éstas son empresas separadas. 
También se supone por simplicidad que al final de cada período " $t$ " las firmas productoras de cobre puede revender el capital comprado a las firmas productoras de bienes de capital.

De esta manera la función objetivo de la empresa que produce cobre es:

$$
\begin{gathered}
\max _{\left\{K_{t+k}^{C U}, L_{t+k}^{C U}, E_{t+k}\right\}_{k=0}^{\infty}} \sum_{k=0}^{\infty} E_{t}\{\beta^{t+k} \Lambda_{t, t+k} \underbrace{\left(P_{t+k}^{C U} A_{t+k}^{C U} L_{t+k}^{C U \alpha} K_{t+k}^{C U \beta} E_{t+k}^{1-\alpha-\beta}+\left(1-\delta^{C U}\right) K_{t+k}^{C U} Q_{t+k}^{C U}\right)\left(1-t_{t}^{u}\right)}_{\text {INGRESOS }}\} \\
\quad-\sum_{k=0}^{\infty} E_{t}\{\beta^{t+k} \Lambda_{t, t+k} \underbrace{\left(R_{F, t+k} Q_{t+k-1}^{C U} K_{t+k}^{C U}+W_{t+k}^{C U} L_{t+k}^{C U}+P_{t+k}^{E} E_{t+k}\right)\left(1-t_{t}^{u}\right)}_{\text {COSTOS }}\}
\end{gathered}
$$

Donde $\beta^{t+k} \Lambda_{t, t+k}$ es el factor estocástico de descuento, $\delta^{C U}$ la tasa de depreciación, $P_{t+k}^{C U}$ el precio del cobre, $W_{t+k}^{C U}$ los salarios del sector, $P_{t+k}^{E}$ el precio de la energía (un índice compuesto de los precios del petróleo y de la energía eléctrica), $Q_{t+k}^{C U}$ el precio del capital, $t_{t}^{u}$ los impuestos a los beneficios y $R_{F, t+k}$ es el retorno del capital. De la solución de (1.9) se obtienen las demandas de capital, trabajo y energía total.

Por otro lado, las firmas productoras de capital compran el capital usado a las firmas productoras de bienes intermedios, reparan el capital depreciado, y construyen nuevo capital, donde $I_{t}^{c u}$ es el nuevo capital creado. Luego, el problema de maximización de las firmas productoras de capital es:

$$
\max _{\left\{I_{t+k}^{C U}\right\}_{k=0}^{\infty}} \sum_{k=0}^{\infty} E_{t}\left\{\Lambda_{t, t+k}\left(\left(Q_{t+k}^{C U}-1\right) I_{t+k}^{C U}-t_{t}^{u} Q_{t+k}^{C U}-f\left(\frac{I_{t+k}^{C U}}{I_{t+k-1}^{C U}}\right) I_{t+k}^{C U}\right)\right\}
$$

Donde $f$ es una función creciente que representa los costos de ajuste de la inversión y $K_{t+k+1}^{C U}=\left(1-\delta^{C U}\right) K_{t+k}^{C U}+I_{t+k}^{C U}$. De la solución de (1.10) se obtiene la oferta de capital, que en conjunto con la demanda de capital (de 1.9) permite determinar el precio del capital y el capital disponible para el próximo período. 
Tercero, al igual que en el resto de la economía se asume que existe rigidez parcial de los salarios (a la Calvo, ver los detalles en García y González 2014). En otras palabras, los salarios van cambiando a través del tiempo en forma exógena por dos fuentes. La fracción de salarios que se reajusta directamente por cambios en los contratos (definida por xi_w_COBR) y, la otra fracción de salarios (definida por index_w_COBRE), que siguen vigentes pero que se reajustan según la inflación pasada.

De la modelación de los salarios, se puede derivar una oferta de trabajo. Así, con este supuesto sobre los salarios, más la ecuación de demanda de trabajo proveniente de (1.8), se obtiene el empleo y los salarios del sector minero. Sólo por motivos de simplicidad, se asume que la utilidad marginal del consumo las familias que trabajan en el sector minero es igual a la utilidad marginal del resto de las familias de la economía.

\section{ii.) El Sector Minero y el Equilibrio General de la Economía.}

En el modelo DSGE estándar de García y González (2014) la única conexión entre el sector minero y el resto de la economía se expresa a través del sector fiscal: una fracción del PIB del cobre se contabiliza directamente como ingresos fiscales; el resto son remesas al exterior.

En el presente estudio, por el contrario, se permite una conexión más amplia, ya que se asume de modo adicional que el sector minero también demanda bienes del resto de la economía, además de la energía eléctrica. Para ilustrar este punto, la ecuación (1.11) representa el equilibrio del mercado de bienes domésticos del modelo estándar:

$$
P_{m, t} Y_{t}=P_{t} C_{t}+P_{t} I_{t}+P_{t} G_{t}+P_{t} X_{t}
$$

Donde $I_{t}$ es la inversión de bienes domésticos o intermedios (no commodities), $Y_{t}$ es la producción de estos bienes, $C_{t}$ es el consumo de los hogares, $X_{t}$ son las exportaciones (demanda externa) y $G_{t}$ es el gasto del gobierno por estos bienes.

A diferencia de lo señalado, en el presente estudio se asume que la inversión del sector minero $I_{t}^{c u}$ se lleva a cabo en el mercado de bienes domésticos: 


$$
P_{m, t} Y_{t}=\underbrace{P_{t} C_{t}+P_{t} I_{t}+P_{t} G_{t}}_{\text {RESTO DE LA ECONOMÍA }}+\underbrace{P_{t} X_{t}}_{\text {SECTOR EXTERNO }}+\underbrace{P_{t} I_{t}^{c u}}_{\text {MINERÍA }}
$$

Por último, una vez agregadas cada una de las restricciones de las familias y las firmas, abstrayéndose de la producción de energía eléctrica para la minería, y considerando que el PIB minero $\left(Q C U_{t}\right)$ se exporta completamente, se obtiene la restricción total de la economía:

$$
\begin{aligned}
& \underbrace{P_{t} C_{t}+P_{t} I_{t}+P_{t} G_{t}+P_{t} I_{t}^{C U}}_{\text {GASTO }}+\underbrace{P_{t} C A_{t}}_{\begin{array}{c}
\text { COSTOS } \\
\text { DE } \\
\text { AIUSTES } \\
\text { INVERSIÓN }
\end{array}} \leq \underbrace{P_{m, t} Y_{t}}_{\begin{array}{c}
\text { PRODUCCIÓN } \\
\text { BIENES } \\
\text { INTERMEDIOS }
\end{array}} \underbrace{-S X_{t} M_{t}-S X_{t} P_{t}^{\text {OIL OIL }} L_{t}-S X_{t} P_{t}^{\text {OIL OIL }} \text { OILU }_{t}^{C U}}_{\begin{array}{c}
\text { IMPORTACIONES } \\
\text { INSUMOS Y COMBUSTIBLES }
\end{array}} \\
& \underbrace{+S X_{t} \frac{B_{t+1}^{*}}{\tilde{R}_{t}^{*}}-S_{t} B_{t}^{*}}_{\text {AMBIO EN LA DEUDA EXTERNA }}+\underbrace{\Gamma\left(S X_{t} P_{t}^{c u} Q C U_{t}\right)}_{\begin{array}{c}
\text { INGRESOS DEL } \\
\text { COBRE }
\end{array}}
\end{aligned}
$$

Donde $S X_{t}$ es el tipo de cambio nominal, $P_{t}^{\text {OIL }}$ el precio del petróleo, $M_{t}$ las importaciones de insumos para la producción de bienes intermedios, $B_{t}^{*}$ la deuda externa, $\tilde{R}_{t}^{*}$ la tasa de interés externa ajustada por premio por riesgo, y $C A_{t}$ son los costos de ajuste de la inversión.

En resumen, los gastos de la economía, incluidos los costos de ajustes de la inversión, se financian con la producción de bienes intermedios, neto de importaciones de los insumos para los bienes intermedios y combustibles (incluido la fracción para el cobre), más el cambio en el financiamiento externo (cambios en la deuda externa) y los ingresos del cobre (PIB cobre menos las remesas al exterior). 


\section{ESTIMACIÓN DEL MOdELO: ReSULTAdOS PRINCIPALES}

En este capítulo se mostrarán los detalles de los resultados de las estimaciones y simulaciones que permiten medir, no sólo el impacto de la minería cobre sobre el resto de la economía, sino también cómo este sector en particular es afectado por variables claves como el precio del cobre, el precio de la energía eléctrica y los salarios.

Los ejercicios que se llevan a cabo son de tres tipos. Primero, se analiza cómo un shock de 1\% en el precio del cobre afecta las variables macroeconómicas de la economía chilena (elasticidad precio del sector minero). Segundo, se mide la contribución a la varianza del crecimiento del PIB de diferentes shocks económicos, entre ellos el precio del cobre y la disponibilidad del mineral. Finalmente, se analizan escenarios contra-factuales, es decir, se adicionan los efectos sobre el sector minero de aumentos de $1 \%$ del precio de la energía, de los salarios reales y de la productividad. Además, se analiza cuál sería el impacto del precio del cobre sobre el resto de la economía, en caso de que la minería fuera un enclave, es decir, si este sector no estuviera integrado al resto de la economía y por lo tanto no demandara bienes intermedios de ella.

\section{VI.1 RESULTADOS ESTIMACIÓN MODELO DSGE14}

La forma correcta de estimar modelos macro DSGE es con econometría bayesiana, por lo cual, se deben establecer priors sobre la distribución de los parámetros (valores priors) y luego a través de técnicas econométricas estándares (máxima verosimilitud) y réplicas (simulación) se obtienen las distribuciones de los posteriores o estimaciones finales. Los priors de los parámetros estimados fueron tomados de la literatura tradicional de modelos macro (ver García y González 2014 y García et al 2013) ${ }^{15}$. Para asegurarse de la calidad de las estimaciones, se realizan dos estimaciones independientes con un número alto de réplicas y se observa que en ambas la distribución de los parámetros converjan a valores similares (ver Anexo $\mathrm{N}^{\circ} 3$ ).

La estrategia de estimación del modelo macro consideró dos partes. Una primera, en que se calibran aquellos parámetros relacionados con el estado estacionario, y una segunda, en que se

\footnotetext{
${ }^{14}$ Lo datos empleados se presentan en el Anexo $\mathrm{N}^{\circ} 2$.

${ }^{15}$ Los posteriores resultantes fueron obtenidos usando el algoritmo de Metropolis-Hastings basado en una cadena de Markov de 20.000 réplicas para construir la distribución estimada de los parámetros (posteriores).
} 
estiman sólo los parámetros relacionados con la dinámica del modelo, es decir, cómo el modelo converge al estado estacionario después de un shock.

En la calibración se replicó el estado estacionario o equilibrio de largo plazo de la economía chilena, medido, por ejemplo, por la razones tales como consumo a PIB, inversión a PIB o gasto de gobierno a PIB, entre otras. En el proceso de calibración es clave obtener los valores para los parámetros de la función de producción del sector minero (ecuación (1.6) $)^{16}$. Estos parámetros representan las participaciones de cada uno de los insumos sobre la producción de cobre. En la calibración de estos parámetros, se utilizó información proveniente de COCHILCO, CNE y Banco Central de Chile. Los resultados de la calibración se muestran en la Tabla 8, en que la participación del capital (51\%) y el trabajo (39\%) son mayoritarios en la producción de cobre. Por otro lado, la energía mayoritaria usada en la producción de cobre es la energía eléctrica (70\%).

\section{Tabla 8: Participación de los Insumos en la Producción de Cobre y la Energía del Sector Minero}

\begin{tabular}{l|c}
\multicolumn{1}{c}{ Parámetros } & Participación \\
\hline Trabajo & 0.39 \\
Capital & 0.51 \\
Energía & 0.1
\end{tabular}

\begin{tabular}{l|c}
\multicolumn{1}{c}{ Parámetros } & Participación \\
\hline Combustibles & 0.3 \\
Energía Eléctrica & 0.7
\end{tabular}

Fuente: cálculo de los autores en base a información de COCHILCO, CNE y BCCh.

En definitiva, la calibración del modelo DSGE produce el siguiente estado estacionario o equilibrio de largo plazo para la economía Chilena, que es coherente con la información que se dispone del Banco Central de Chile (ver Tabla 9).

\footnotetext{
16 En el Anexo № 3 se presenta los parámetros para el resto de la economía.
} 


\section{Tabla 9: estado estacionario del modelo DSGE}

\begin{tabular}{l|c}
\multicolumn{1}{c}{ Estado Estacionario } & razón sobre PIB \\
\hline Consumo & 0.64 \\
Inversión Intermedios & 0.19 \\
Inversión Cobre & 0.06 \\
Gasto de Gobierno & 0.10 \\
Exportaciones Intermedios & 0.27 \\
Importaciones Insumos & 0.41 \\
Importaciones Combustibles & 0.03 \\
PIB Cobre & 0.17 \\
Carga Tributaria & 0.18
\end{tabular}

Fuente: cálculo de los autores en base al modelo macro DSGE.

A continuación se analizarán los parámetros asociados con el impacto de la minería de cobre ${ }^{17}$ sobre la economía (ver Tabla 10). En primer lugar, se observan las desviaciones estándar de los shocks que afectan a este sector. Al respecto, la mayor volatilidad proviene del propio precio del cobre y el petróleo, ambos en tornos al 16\% (siendo el petróleo un insumo importante en la producción de cobre). Asimismo, se tiene que estas dos variables representan en términos absolutos los shocks más volátiles que enfrenta la economía Chilena según la estimación del modelo DSGE (ver Anexo $\mathrm{N}^{\circ} 3$ ). En seguida está el efecto del precio de la energía eléctrica y del shock que mide la disponibilidad del material (de cobre), con magnitudes más moderadas y comparables a otros shocks que afectan a la economía, es decir, en torno al 6\% (ver Anexo $\mathrm{N}^{\circ} 3$ ).

A continuación, como se explicó con la ecuación (1.8), los parámetros (pmg_s's) miden en el corto plazo la sensibilidad de las demandas de cada insumo a la actividad y los precios en el sector minero. Como se observa en la Tabla 10 todos los parámetros pmg_'s están muy por debajo de 0.5, indicando una fuerte inercia en el sector minero de cobre en lo que respecta a la contratación de nuevos insumos. Es decir, dentro de un trimestre, la decisión de demandar un insumo está fuertemente influenciada por la decisión que se tomó en el período anterior, y el proceso de ajuste entendido como el tiempo que se toma en cambiar completamente la decisión de demanda de un

\footnotetext{
${ }^{17}$ En relación a las estimaciones de los parámetros que determinan la dinámica del modelo macro, se observa que en general muchos de los valores estimados están en línea con los valores encontrados en otros estudios (García y González, 2013 y García et al 2013). En el Anexo N 3 se presenta en detalle las estimaciones de todos los parámetros del modelo macro.
} 
insumo por un cambio en los precios y en el nivel de producción, dura en promedio cinco trimestres ${ }^{18}$.

En lo relativo a los salarios en el sector minero, según el modelo éstos permanecen rígidos en torno a un año ${ }^{19}$, y presentan una indexación a la inflación pasada cercana a uno ${ }^{20}$. Este resultado está en línea con los obtenidos en el marco de la estimación de la dinámica de los salarios en el resto de la economía (ver Anexo $N^{\circ} 3$ )

Por último, todos los shocks que enfrenta este sector tienen una persistencia, (parámetros rho_s) ${ }^{21}$ por sobre el 0,8 .

Tabla 10: parámetros relacionados con el impacto de la minería de cobre sobre la economía.

\begin{tabular}{|c|c|c|c|c|c|c|}
\hline Parámetros & $\begin{array}{l}\text { Prior } \\
\text { Media }\end{array}$ & $\begin{array}{l}\text { Posterior } \\
\text { Media }\end{array}$ & \multicolumn{2}{|c|}{$\begin{array}{c}\text { Intervalo de } \\
\text { Confianza 90\% }\end{array}$} & $\begin{array}{c}\text { Distribución } \\
\text { Prior }\end{array}$ & $\begin{array}{l}\text { Desviación } \\
\text { Estándar Prior }\end{array}$ \\
\hline pmg_EE_EN_COBRE & 0.5 & 0.2564 & 0.1317 & 0.3676 & beta & 0.1 \\
\hline pmg_EN_CŌBRE & 0.5 & 0.1423 & 0.0555 & 0.2258 & beta & 0.1 \\
\hline pmg_L_COBRE & 0.5 & 0.0848 & 0.051 & 0.1188 & beta & 0.1 \\
\hline pmg_K_COBRE & 0.5 & 0.5461 & 0.4031 & 0.6778 & beta & 0.1 \\
\hline index_w_t_COBRE & 0.9 & 0.9046 & 0.8335 & 0.9712 & beta & 0.05 \\
\hline xi_w_COBRE & 0.67 & 0.6216 & 0.5705 & 0.6813 & beta & 0.05 \\
\hline rho_ōoil & 0.9 & 0.8655 & 0.7959 & 0.9337 & beta & 0.05 \\
\hline rho_Pcu & 0.9 & 0.8623 & 0.8377 & 0.8915 & beta & 0.05 \\
\hline rho_A_COBRE & 0.9 & 0.9045 & 0.8917 & 0.9168 & beta & 0.01 \\
\hline rho_PEE & 0.5 & 0.8518 & 0.8016 & 0.9022 & beta & 0.1 \\
\hline Desviación estándar: & & & & & & \\
\hline Precio del Cobre & 16.53 & 16.627 & 15.8637 & 17.4668 & invg2 & 0.5 \\
\hline Precio del Petróleo & 16.07 & 16.2808 & 15.608 & 17.0067 & invg2 & 0.5 \\
\hline Precio Salarios M ineros & 0.9 & 0.5559 & 0.4213 & 0.6845 & invg2 & 0.5 \\
\hline Precio Energía Eléctrica & 6.84 & 6.9106 & 6.2696 & 7.6251 & invg2 & 0.5 \\
\hline $\mathrm{A}^{\mathrm{cu}}$ & 3.59 & 7.2804 & 5.834 & 8.6681 & $\operatorname{invg} 2$ & 0.5 \\
\hline
\end{tabular}

Fuente: cálculo de los autores usando el modelo DSGE.

183.9 trimestres $=1 /(1-0,74)$, donde 0,74 es el rezago promedio de los parámetros ( 1 - pmg_'s.)

192.65 trimestres $=1 /\left(1-\mathrm{xi}_{-} \mathrm{w}_{-} \mathrm{COBRE}\right)=1 /(1-0,62)$.

20 index_w_COBRE $=0,90$.

${ }^{21}$ Las filas que representan parámetros rho, muestran en la segunda columna (Posterior Media) la persistencia del cambio porcentual de la variable de que se trate, en el tiempo. De este modo, por ejemplo, si el "Posterior Media" del rho del precio de cobre es 0,862 , ello implica que en cada trimestre el cambio en el precio se mantendrá en el trimestre siguiente en un $86,2 \%$. 


\section{VI.2 EFECTOSDEL SECTOR Minero}

Con el objeto de medir el impacto del sector minero sobre la economía Chilena se optó por analizar el efecto a través del tiempo (trimestres) de un shock en el precio del cobre de un $1 \%$, sobre todas las variables de la economía, suponiendo que ningún otro shock la está afectando. Esta forma de cuantificar el impacto se conoce como análisis de impulso y respuesta (IRF), y es la manera estándar no sólo de cuantificar un efecto en modelos macroeconómicos sino de también de poder entender cómo un efecto particular se esparce o distribuye dentro de la economía, es decir, de tener la capacidad contar una "historia" consistente del fenómeno desde una perspectiva económica. Dicha "historia" puede confirmar la intuición que se tiene sobre un fenómeno económico particular o clarificar con nueva información las reales características de dicho fenómeno y su efecto sobre la economía22.

Se presentará la información a través de un gráfico (trimestral) para definir la forma en que el precio del cobre afecta el resto de la economía, es decir, la historia que está detrás de un cambio en el precio del cobre. El gráfico está compuesto de sub-gráficos donde se muestra la evolución de las diferentes variables macroeconómicas a través de los trimestres, después de un shock del precio del cobre de un $1 \%$. Para poder comparar entre variables, todos los sub-gráficos tienen las mismas dimensiones en el eje vertical.

Luego en una tabla se resumirá de manera precisa el impacto del precio del cobre en las principales variables macroeconómicas a través de los años. Puesto que el modelo DSGE es lineal y fue estimado con datos en cambios porcentuales (diferencias de log multiplicados por 100), los números de la tabla pueden interpretarse como elasticidades. De este modo, si se quiere saber cuál sería el efecto de un incremento de un 10\% en el precio del cobre, hay que multiplicar por 10 los números presentados en la tabla. Con este método es fácil cuantificar cualquier impacto sobre la economía Chilena de cambio en el precio del cobre.

El Gráfico 8 muestra los impulsos-respuestas de la economía a un shock en el precio del cobre de un $1 \%$. En este contexto, es importante destacar que el aumento inesperado de un $1 \%$ del precio del cobre en un trimestre tiene un efecto que subsiste a través del tiempo, no obstante va desapareciendo paulatinamente a través de los trimestres. Esto ocurre por la alta persistencia que tienen los cambios del precio del Cobre en el tiempo -con un valor 0,86 en cada trimestre-, lo que

\footnotetext{
22 En adelante, debe entenderse que un shock por una sola vez está definido en "variaciones porcentuales".
} 
también sucede con los shocks de productividad, salario y precio de la energía eléctrica (ver Tabla 10 , parámetros rho_s con valores por sobre 0.8 ).

Claramente, el shock analizado es expansivo, es decir, produce un aumento del crecimiento del PIB, la inversión, el uso de energía eléctrica, el empleo y los salarios del sector minero. La forma en que se esparce en la economía Chilena es la siguiente, confirmando el análisis de correlaciones presentado al comienzo de este estudio:

- Hay un efecto expansivo directo en el PIB, por ser el PIB minero parte de éste, alcanzando una participación de un 17\% en promedio en los últimos años.

- Se produce un aumento menor en el consumo y una mayor demanda del sector minero por bienes intermedios, lo que impulsa un aumento de los insumos importados y el empleo no cobre (los salarios no cobre prácticamente se mantienen constante). Todo esto fortalece la expansión del PIB total, más allá del crecimiento del PIB minero.

- El gasto de gobierno sube moderadamente puesto que se asume que está operando la regla fiscal en el modelo DSGE.

- La inflación por el mayor nivel de actividad sube marginalmente, ocasionando que el Banco Central suba levemente la tasa de interés, lo que produce dos efectos:

- Cae el tipo de cambio real y con ello las exportaciones no cobre.

- Se reduce la inversión no cobre, aunque la inversión total, es decir, la que incluye al sector minero, sube.

Se puede constatar que un aumento marginal de la tasa de interés tiene efectos no menores en el tipo de cambio real y la inversión no cobre, debido a que estas variables en el modelo DSGE no dependen sólo del valor actual de la tasa de interés sino también de la trayectoria completa de dicha variable (tasa de interés de largo plazo). La acumulación de tasas marginales más altas por un período prolongado reduce finalmente estas dos variables en los primeros períodos. 
Para visualizar lo indicado de modo más preciso, la Tabla 11 muestra los valores en los crecimientos de las diferentes variables macroeconómicas por un shock del precio del Cobre de un 1\%. Esta tabla está construida con la misma información del Gráfico 8, pero resume la información en términos anuales. Se detalla la evolución de la economía hasta cinco años después del shock, y además se entregan los resultados acumulados para 1, 5 y 10 años. Los principales cambios observados para el caso de cambios acumulados en cinco años son:

- El PIB crece después de 5 años de ocurrido el shock en hasta 0,16\% por un aumento de un $1 \%$ del precio del cobre. De esta forma la elasticidad por aumento del precio del cobre es $0,16=(0.16 \% / 1 \%)$ en cinco años.

- Ese crecimiento en el PIB agregado es impulsado mayoritariamente por una aumento de $0.192 \%$ en el crecimiento del PIB Minero en ese lapso de tiempo.

- En la industria del cobre, la inversión crece fuertemente durante el primer año $(0,91 \%)$, pero luego decrece. El aumento de la producción causa aumentos del empleo cobre $(0,23 \%)$ y del salario $(0,13 \%)$, y un aumento de la demanda de energía eléctrica $(0,38 \%)$ en cinco años.

- Considerando que el precio del cobre muestra fuertes desviaciones estándar de hasta 16\% , una fluctuación positiva de esta magnitud correspondería a casi dos puntos de crecimiento del PIB en 5 años $(2,56 \%=16 * 0,16)$. En otras palabras, una desviación de un $16 \%$ en el precio del cobre, llevará en cinco años a un cambio en el PIB de un $2,56 \%$.

- El crecimiento de la inversión cobre más que compensa la caída de la inversión no cobre en el primer año. Sin embargo, al caer la inflación el Banco Central también reduce la tasa de interés recuperándose parcialmente la inversión no cobre.

- Si bien el consumo sube en 0,015\% en cinco años, el principal agregado macro que aumenta es el gasto de gobierno $(0,088 \%)$.

- El aumento del precio del cobre sin duda que está asociado a una caída del tipo de cambio real, que cae en $-0,044 \%$, afectando a las exportaciones no cobre en $-0.036 \%$ 
en cinco años. No obstante, durante ese mismo período el empleo no cobre sube hasta en $0,026 \%$ en cinco años.

\section{Gráfico 8: Efecto del Precio del Cobre en la Economía Chilena, todas las variables medidas como tasas de crecimiento.}
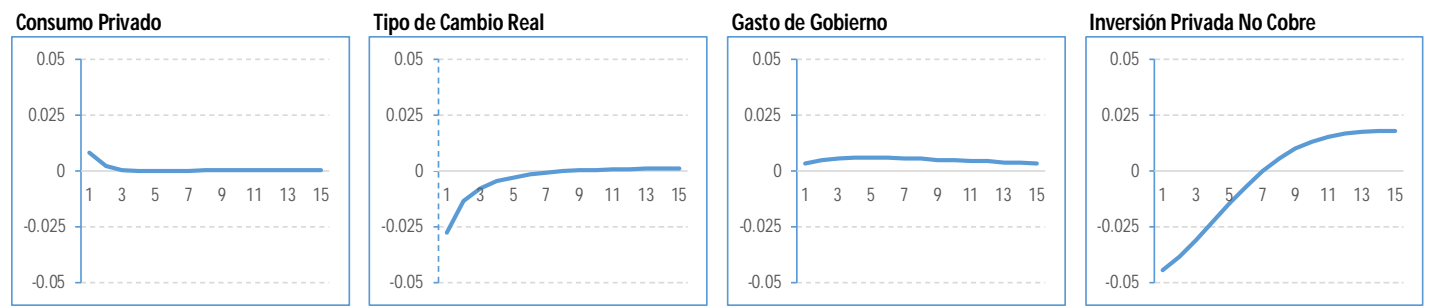

Empleo No Cobre

Importaciones No Petróleo

PIB
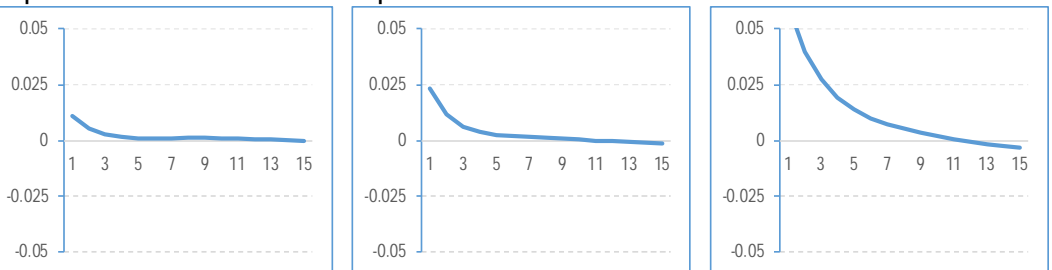

Salarios No Cobre

Exportaciones No Cobre

Tasa de Política Monetaria

Inflación
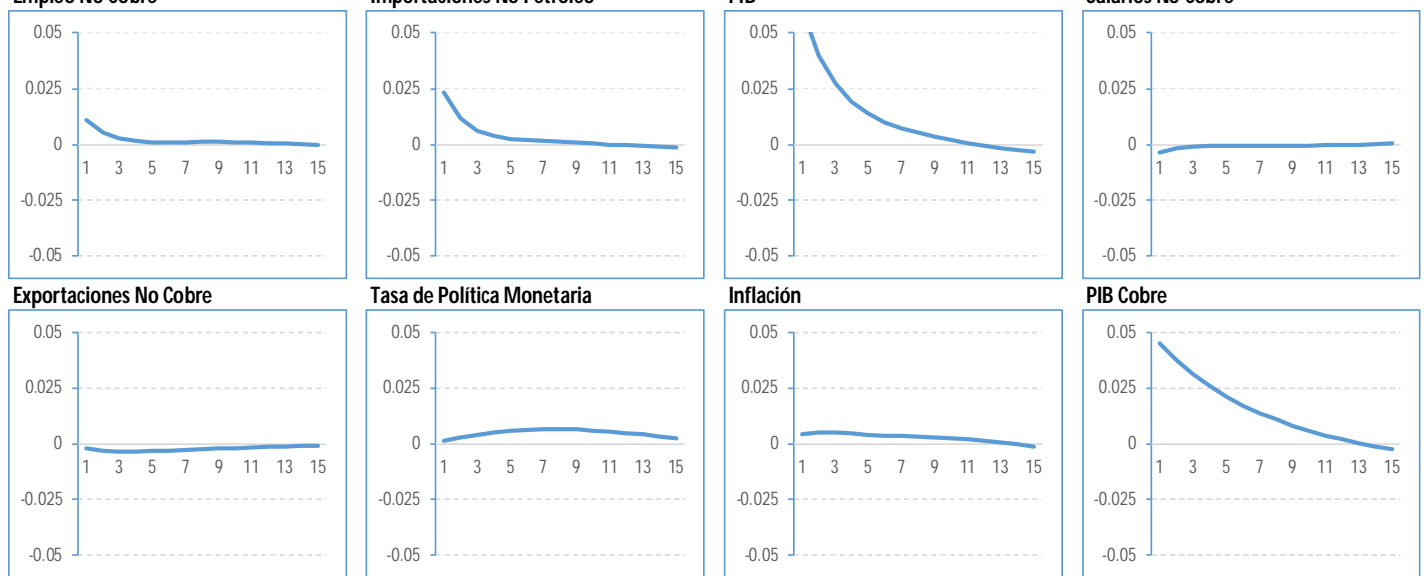

Inversión Cobre

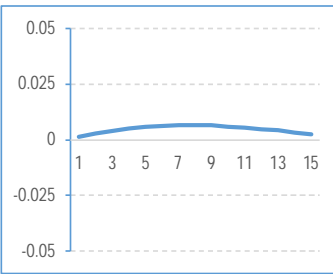

Empleo Cobre

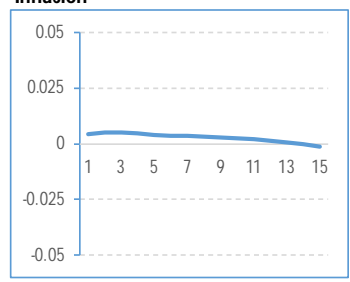

PIB Cobre

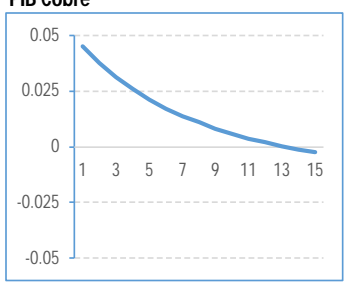

Salarios Cobre

Energía Electrica Cobre
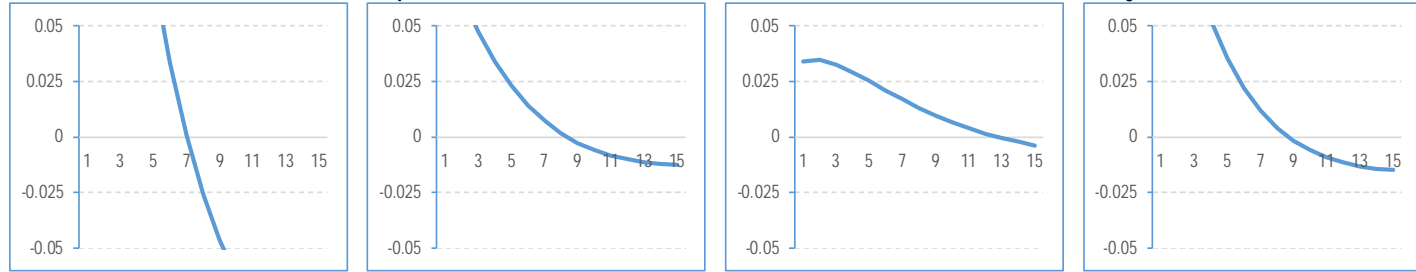

Fuente: Los autores en base al modelo DSGE. 
Tabla 11: Impacto de un aumento de un $1 \%$ en el precio del cobre.

\begin{tabular}{|c|c|c|c|c|c|c|c|c|c|c|c|c|c|c|c|c|}
\hline Años & $\mathrm{C}$ & $E$ & $\mathrm{G}$ & INC & ENC & MNP & PIB & W & $\bar{X}$ & R & PI & PIBC & IC & EC & WC & $\mathrm{EE}$ \\
\hline 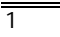 & 0.011 & -0.054 & 0.020 & -0.137 & 0.020 & 0.045 & 0.147 & -0.007 & -0.012 & 0.013 & 0.019 & 0.140 & 0.911 & 0.229 & 0.130 & 0.381 \\
\hline 2 & 0.000 & -0.004 & 0.023 & -0.016 & 0.004 & 0.007 & 0.036 & -0.002 & -0.012 & 0.025 & 0.014 & 0.063 & 0.083 & 0.046 & 0.076 & 0.073 \\
\hline 3 & 0.001 & 0.003 & 0.019 & 0.055 & 0.004 & 0.001 & 0.006 & -0.002 & -0.007 & 0.023 & 0.008 & 0.020 & -0.267 & -0.027 & 0.022 & -0.028 \\
\hline 4 & 0.001 & 0.005 & 0.015 & 0.072 & 0.000 & -0.004 & -0.011 & 0.001 & -0.004 & 0.011 & -0.003 & -0.007 & -0.364 & -0.048 & -0.011 & -0.057 \\
\hline 5 & 0.001 & 0.006 & 0.011 & 0.062 & -0.003 & -0.006 & -0.019 & 0.004 & -0.001 & -0.007 & -0.017 & -0.023 & -0.331 & -0.048 & -0.027 & -0.060 \\
\hline \multicolumn{17}{|c|}{ Acumulado } \\
\hline 1 & 0.011 & -0.054 & 0.020 & -0.137 & 0.020 & 0.045 & 0.147 & -0.007 & -0.012 & 0.013 & 0.019 & 0.140 & 0.911 & 0.229 & 0.130 & 0.381 \\
\hline 5 & 0.015 & -0.044 & 0.088 & 0.035 & 0.026 & 0.042 & 0.159 & -0.006 & -0.036 & 0.064 & 0.021 & 0.192 & 0.027 & 0.151 & 0.191 & 0.307 \\
\hline \multirow[t]{9}{*}{10} & 0.020 & -0.022 & 0.112 & 0.093 & -0.002 & 0.023 & 0.043 & 0.024 & -0.023 & -0.176 & -0.209 & 0.016 & -0.452 & 0.005 & 0.050 & 0.068 \\
\hline & \multicolumn{4}{|c|}{ Consumo Privado } & $\mathrm{x}$ & \multicolumn{11}{|c|}{ Exportaciones No Cobre } \\
\hline & \multicolumn{4}{|c|}{ Tipo de Cambio Real } & $\mathrm{R}$ & \multicolumn{11}{|c|}{ TPM } \\
\hline & \multicolumn{4}{|c|}{ Gasto de Gobierno } & $\mathrm{PI}$ & \multicolumn{11}{|c|}{ Inflación } \\
\hline & \multicolumn{4}{|c|}{ Inversión Privada No Cobre } & PIBC & \multicolumn{11}{|c|}{ PIB Cobre } \\
\hline & \multicolumn{4}{|c|}{ Empleo No Cobre } & IC & \multicolumn{11}{|c|}{ Inversión Cobre } \\
\hline & \multirow{2}{*}{\multicolumn{4}{|c|}{$\begin{array}{l}\text { Importaciones No Petróleo } \\
\text { PB }\end{array}$}} & $\mathrm{EC}$ & \multicolumn{11}{|c|}{ Empleo Cobre } \\
\hline & & & & & WC & \multicolumn{11}{|c|}{ Salarios Reales Cobre } \\
\hline & \multicolumn{4}{|c|}{$\begin{array}{l}\text { PIB } \\
\text { Salarios Reales No Cobre }\end{array}$} & $\mathrm{EE}$ & \multicolumn{11}{|c|}{ Energía Eléctrica Cobre } \\
\hline
\end{tabular}

Fuente: Los autores basado en el modelo DSGE

\section{VI.3 CONTRIBUCión DEL SECTOR MiNERO AL CRECIMIENTO}

Con el objeto de medir la contribución del sector minero cobre al crecimiento se descompone la varianza del crecimiento observado en el período 2003-2013 en los shocks macroeconómicos del modelo DSGE. En consecuencia, la fortaleza de este análisis es considerar todos los shocks en conjunto, y por tanto, poder visualizar con más claridad la importancia de la minería, y en particular del precio del cobre, en comparación a otros elementos que también son causantes del ciclo económico de la economía Chilena.

Por construcción, los mencionados shocks deben contabilizar el $100 \%$ de la varianza del crecimiento de la economía Chilena en el período de tiempo antes definido. Por tanto, en el modelo DSGE se incluyó una gran variedad de shocks estudiados por la literatura de fluctuaciones económicas para economías abiertas (monetarios, fiscales, productivos, términos de intercambio, premio por riesgo, etc.), de manera de no dejar en unos pocos shocks toda la explicación de las fluctuaciones observadas en la economía Chilena. Además, debido a la alta persistencia de los shocks que enfrenta la economía, se realizó el análisis de la descomposición de varianza del crecimiento desde un trimestre hasta veinte trimestres.

El enfoque descrito permite poner en perspectiva el efecto del precio del cobre, no solo sobre el crecimiento del PIB sino también sobre unas de las variables claves de la economía Chilena: el tipo de cambio real. En efecto, de la sección anterior se desprende claramente que un aumento del precio del cobre hace caer el tipo de cambio real y con ello las exportaciones no cobre. Pero en el período 2003-2013 surge una importante pregunta al respecto: ¿ha sido el precio del cobre un 
determinante fundamental de la evolución del tipo cambio real o son otros shocks los que han determinado la evolución de esta variable?

La Tabla 12 indica un elemento clave en la economía nacional: los factores externos son casi tan importantes como la productividad para explicar el ciclo económico. Entre estos factores, el precio del cobre por sí mismo contabiliza cerca del 5,8\% de la varianza del PIB en el período 2003-2013, y es el segundo factor más importante después del premio por riesgo, y por lejos más importante que la actividad externa (crecimiento ponderado de EE.UU., Europa y Japón), las tasas de interés externa y el precio del petróleo.

Más aún, la Tabla 13 indica que el precio del cobre es muy marginal explicando las fluctuaciones del tipo de cambio real. Por el contrario, el premio por riesgo y la productividad explican casi el $60 \%$ de la fluctuación de esta variable. Por tanto, se puede afirmar que si bien un mayor precio del cobre produce una apreciación real del peso, las fluctuaciones del tipo de cambio en el período 2003-2013 han estado asociadas a otros shocks de tipo más financieros y de productividad. 23

\section{Tabla 12: Descomposición de Varianza del Crecimiento del PIB}

\begin{tabular}{lccccc} 
Crecimiento del PIB & \multicolumn{2}{c}{ Trimestres } & & & \\
Shocks & 1 & 4 & 8 & 16 & 20 \\
\hline \hline Consumo & $15.4 \%$ & $25.1 \%$ & $21.0 \%$ & $20.8 \%$ & $20.7 \%$ \\
TPM (M onetario) & $13.5 \%$ & $8.7 \%$ & $8.5 \%$ & $8.4 \%$ & $8.3 \%$ \\
Gasto de Gobierno & $4.5 \%$ & $3.5 \%$ & $2.8 \%$ & $2.8 \%$ & $2.7 \%$ \\
Premio por Riesgo & $2.8 \%$ & $2.4 \%$ & $2.0 \%$ & $2.0 \%$ & $2.0 \%$ \\
Salarios & $46.8 \%$ & $33.3 \%$ & $32.9 \%$ & $32.7 \%$ & $32.7 \%$ \\
Tasa de Interés Externa & $0.3 \%$ & $0.5 \%$ & $0.5 \%$ & $0.6 \%$ & $0.6 \%$ \\
Pib Externo & $0.5 \%$ & $0.4 \%$ & $0.5 \%$ & $0.6 \%$ & $0.6 \%$ \\
Precio Cobre & $6.0 \%$ & $5.8 \%$ & $5.0 \%$ & $4.9 \%$ & $5.0 \%$ \\
Precio Petróleo & $0.4 \%$ & $0.5 \%$ & $0.6 \%$ & $0.7 \%$ & $0.7 \%$ \\
Productividad & $0.8 \%$ & $14.8 \%$ & $22.0 \%$ & $22.5 \%$ & $22.6 \%$ \\
Disponibilidad Cobre & $0.6 \%$ & $0.5 \%$ & $0.4 \%$ & $0.4 \%$ & $0.4 \%$ \\
Otros & $8.4 \%$ & $4.6 \%$ & $3.7 \%$ & $3.6 \%$ & $3.6 \%$ \\
& & & & & \\
Minero (precio cobre + disponibilidad) & $\mathbf{6 . 7 \%}$ & $\mathbf{6 . 3} \%$ & $\mathbf{5 . 4 \%}$ & $\mathbf{5 . 4 \%}$ & $\mathbf{5 . 4 \%}$ \\
Factores Externos & $10.0 \%$ & $9.6 \%$ & $8.7 \%$ & $8.9 \%$ & $8.9 \%$
\end{tabular}

${ }^{23}$ Al respecto, García y González (2013) encuentran que este fenómeno se repite en otras economías con un importante sector minero como Australia. 


\section{Tabla 13: Descomposición de Varianza del Cambio (\%) del tipo de cambio real bilateral con los EE.UU.}

\begin{tabular}{lccccc} 
Cambios del Tipo de Cambio Real & \multicolumn{2}{c}{ Trimestres } & & & \\
Shocks & 1 & 4 & 8 & 16 & 20 \\
\hline \hline Consumo & $10.5 \%$ & $14.6 \%$ & $14.1 \%$ & $14.1 \%$ & $14.1 \%$ \\
TPM (M onetario) & $4.0 \%$ & $3.5 \%$ & $3.6 \%$ & $3.6 \%$ & $3.6 \%$ \\
Gasto de Gobierno & $0.0 \%$ & $0.0 \%$ & $0.0 \%$ & $0.0 \%$ & $0.0 \%$ \\
Premio por Riesgo & $23.2 \%$ & $26.6 \%$ & $25.6 \%$ & $25.5 \%$ & $25.5 \%$ \\
Salarios & $15.2 \%$ & $13.3 \%$ & $13.6 \%$ & $13.6 \%$ & $13.6 \%$ \\
Tasa de Interés Externa & $9.5 \%$ & $8.6 \%$ & $8.7 \%$ & $8.7 \%$ & $8.7 \%$ \\
Pib Externo & $1.9 \%$ & $1.6 \%$ & $1.6 \%$ & $1.6 \%$ & $1.6 \%$ \\
Precio Cobre & $0.4 \%$ & $0.0 \%$ & $0.0 \%$ & $0.0 \%$ & $0.0 \%$ \\
Precio Petróleo & $0.1 \%$ & $0.2 \%$ & $0.2 \%$ & $0.2 \%$ & $0.2 \%$ \\
Productividad & $35.2 \%$ & $31.1 \%$ & $32.3 \%$ & $32.2 \%$ & $32.2 \%$ \\
Disponibilidad Cobre & $0.0 \%$ & $0.0 \%$ & $0.0 \%$ & $0.0 \%$ & $0.0 \%$ \\
Otros & $0.0 \%$ & $0.4 \%$ & $0.4 \%$ & $0.4 \%$ & $0.4 \%$ \\
& & & & & \\
Minero (precio cobre +disponibilidad) & $\mathbf{0 . 4 \%}$ & $\mathbf{0 . 0 \%}$ & $\mathbf{0 . 0} \%$ & $\mathbf{0 . 0} \%$ & $\mathbf{0 . 0} \%$ \\
Premio por Riesgo & $23.2 \%$ & $26.6 \%$ & $25.6 \%$ & $25.5 \%$ & $25.5 \%$
\end{tabular}

Fuente Tablas 14 y 15: Los autores, basado en el modelo DSGE

\section{VI.4 ANÁLISIS DE ESCENARIOS}

En esta sección se analiza qué sucedería si ocurren los siguientes escenarios alternativos o contra-factuales:

- El precio de la energía eléctrica sube exógenamente en un $1 \%$ en un trimestre y luego decrece con un factor de persistencia de 0,85 por trimestre.

- Los salarios reales del sector crecen exógenamente en un $1 \%$ en un trimestre y luego decrecen lentamente, puesto que la rigidez salarial es de casi 3 trimestres (ver nota al pie 19, para su cálculo) y que la inercia salarial a la inflación es de 0,9 por trimestre (Tabla 10, parámetro index_w_cobre).

- La productividad del sector crece exógenamente en un $1 \%$ en un trimestre y luego decrece con un factor de persistencia de 0,9 por trimestre.

- Se supone que la industria de la minería del cobre no está integrada o conectada al resto de la economía Chilena a través de la demanda de insumos intermedios, sino que se comporta como un "enclave" cuyo aporte se reduce a aportar a los ingresos fiscales (directos, a través de CODELCO, y por impuestos a las utilidades).

Con el objetivo de aislar los efectos de cada uno de estos escenarios de otros shocks, se usa el análisis de impulso respuesta presentada en la Tabla 11. La Tabla 14 indica que la demanda de 
energía eléctrica cae en un 0,4, impactando negativamente en el PIB minero en un - $0.031 \%$ y el PIB de la economía en $-0.027 \%$, durante el primer año. Luego el efecto decrece llegando a un poco más de la mitad de este porcentaje en cinco años. Este ejercicio ilustra el importante impacto sobre la industria del cobre y de la economía como un todo del encarecimiento progresivo de la energía eléctrica.

La Tabla 15 indica lo que ocurriría si los salarios suben arbitrariamente en un 1\% en un trimestre. Puesto que este insumo corresponde al 39\% del valor de la producción de cobre, el impacto negativo sobre el PIB minero es fuerte, alcanzando un $-0,161 \%$ el primer año. Con esto el PIB de la economía cae en un -0,139\%, durante el primer año. Luego, el efecto disminuye llegando hasta una caída de -0,137\% en cinco años en el PIB minero.

En este ejercicio queda claro el impacto negativo de aumentos salariales por encima de la productividad (disponibilidad del mineral) y el precio del cobre, no solo en la industria minera sino en toda la economía. Sin embargo, si los salarios se mueven por la productividad esto permite aumentar naturalmente los salarios reales, el empleo y el PIB en el sector, sin la necesidad de generar ningún tipo de contracción (Tabla 16).

El Gráfico 9, por otro lado, ilustra la importancia de modelar adecuadamente la integración de la minería al resto de la economía y no sólo considerar los efectos fiscales de este sector. Si en el modelo DSGE se hubiera considerado el sector minero sólo como un enclave, y no integrado al resto de la economía, los efectos sobre el crecimiento del PIB se deberían dividir casi por 2,5 en el impacto.

Tabla 14: Impacto de un aumento de un $1 \%$ en el precio de la energía eléctrica.

\begin{tabular}{|c|c|c|c|c|c|c|c|c|c|c|c|c|c|c|c|c|}
\hline Años & C & $\mathrm{E}$ & G & INC & ENC & MNP & PIB & $W$ & $\mathrm{X}$ & $\mathrm{R}$ & $\mathrm{PI}$ & PIBC & IC & EC & WC & $\mathrm{EE}$ \\
\hline 1 & -0.001 & 0.004 & -0.001 & $\overline{0.010}$ & -0.002 & -0.003 & $=0.027$ & 0.001 & 0.001 & -0.001 & -0.002 & -0.031 & -0.067 & -0.005 & $=0.004$ & -0.400 \\
\hline 2 & 0.000 & 0.001 & -0.001 & 0.004 & 0.000 & -0.001 & -0.003 & 0.000 & 0.001 & -0.003 & -0.003 & -0.005 & -0.020 & -0.005 & -0.004 & 0.004 \\
\hline 3 & 0.000 & 0.000 & -0.001 & -0.002 & 0.000 & 0.000 & 0.002 & 0.000 & 0.001 & -0.003 & -0.002 & 0.002 & 0.009 & -0.003 & -0.003 & 0.086 \\
\hline 4 & 0.000 & 0.000 & -0.001 & -0.005 & 0.000 & 0.000 & 0.003 & 0.000 & 0.000 & -0.003 & -0.002 & 0.003 & 0.023 & -0.001 & -0.002 & 0.084 \\
\hline 5 & 0.000 & 0.000 & -0.001 & -0.005 & 0.000 & 0.000 & 0.003 & 0.000 & 0.000 & -0.002 & -0.001 & 0.004 & 0.027 & 0.001 & 0.000 & 0.065 \\
\hline \multicolumn{17}{|c|}{ Acumulado } \\
\hline 1 & 0.00 & 0.00 & 0.00 & 0.01 & 0.00 & 0.00 & -0.03 & 0.00 & 0.00 & 0.00 & 0.00 & -0.03 & -0.07 & -0.005 & 0.00 & -0.40 \\
\hline 5 & 0.00 & 0.00 & -0.01 & 0.00 & 0.00 & 0.00 & -0.02 & 0.00 & 0.00 & -0.01 & -0.01 & -0.03 & -0.03 & -0.013 & -0.01 & -0.16 \\
\hline 10 & 0.00 & 0.00 & -0.01 & 0.00 & 0.00 & 0.00 & -0.02 & 0.00 & 0.00 & -0.01 & -0.01 & -0.03 & -0.03 & -0.012 & -0.01 & -0.16 \\
\hline & C & \multicolumn{4}{|c|}{ Consumo Privado } & $\mathrm{X}$ & \multicolumn{10}{|c|}{ Exportaciones No Cobre } \\
\hline & $E$ & \multicolumn{4}{|c|}{ Tipo de Cambio Real } & $\mathrm{R}$ & \multicolumn{10}{|l|}{ TPM } \\
\hline & G & \multicolumn{4}{|c|}{ Gasto de Gobierno } & $\mathrm{PI}$ & \multicolumn{10}{|c|}{ Inflación } \\
\hline & INC & \multicolumn{4}{|c|}{ Inversión Privada No Cobre } & PIBC & \multicolumn{10}{|c|}{ PIB Cobre } \\
\hline & ENC & \multicolumn{4}{|c|}{ Empleo No Cobre } & IC & \multicolumn{10}{|c|}{ Inversión Cobre } \\
\hline & MNP & \multicolumn{4}{|c|}{ Importaciones No Petróleo } & EC & \multicolumn{10}{|c|}{ Empleo Cobre } \\
\hline & PIB & \multicolumn{4}{|c|}{ PIB } & WC & \multicolumn{10}{|c|}{ Salarios Reales Cobre } \\
\hline & W & \multicolumn{4}{|c|}{ Salarios Reales No Cobre } & $\mathrm{EE}$ & \multicolumn{10}{|c|}{ Energía Eléctrica Cobre } \\
\hline
\end{tabular}

Fuente: Los autores basado en el modelo DSGE 


\section{Tabla 15: Impacto de un aumento de un $1 \%$ en el salario real de la minería.}

\begin{tabular}{|c|c|c|c|c|c|c|c|c|c|c|c|c|c|c|c|c|}
\hline Años & $C$ & $E$ & $\bar{G}$ & INC & ENC & MNP & PIB & $\bar{W}$ & $\bar{x}$ & $\mathrm{R}$ & $\mathrm{PI}$ & PIBC & IC & EC & WC & $\mathrm{EE}$ \\
\hline 1 & "-0.004 & 0.017 & -0.003 & 0.057 & -0.008 & -0.015 & -0.139 & 0.003 & 0.004 & -0.007 & -0.011 & -0.161 & "-0.364 & -0.375 & 0.819 & $\overline{-0.047}$ \\
\hline 2 & 0.001 & 0.004 & -0.006 & 0.017 & -0.002 & -0.003 & -0.027 & 0.002 & 0.004 & -0.015 & -0.012 & -0.042 & -0.092 & -0.053 & -0.590 & -0.053 \\
\hline 3 & 0.000 & 0.000 & -0.007 & -0.017 & -0.002 & -0.001 & 0.012 & 0.002 & 0.003 & -0.017 & -0.011 & 0.009 & 0.073 & 0.068 & -0.266 & -0.029 \\
\hline 4 & 0.000 & -0.001 & -0.007 & -0.030 & -0.001 & 0.001 & 0.024 & 0.001 & 0.002 & -0.013 & -0.007 & 0.027 & 0.146 & 0.094 & -0.095 & -0.007 \\
\hline 5 & -0.001 & -0.003 & -0.006 & -0.029 & 0.000 & 0.002 & 0.025 & -0.001 & 0.001 & -0.006 & -0.001 & 0.031 & 0.154 & 0.084 & -0.015 & 0.007 \\
\hline \multicolumn{17}{|c|}{ Acumulado } \\
\hline 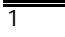 & -0.004 & 0.017 & -0.003 & 0.057 & -0.008 & -0.015 & -0.139 & 0.003 & 0.004 & -0.007 & -0.011 & -0.161 & -0.364 & -0.375 & 0.819 & -0.047 \\
\hline 5 & -0.004 & 0.018 & -0.028 & -0.002 & -0.013 & -0.017 & -0.106 & 0.007 & 0.014 & -0.059 & -0.042 & -0.137 & -0.084 & -0.183 & -0.153 & -0.129 \\
\hline 10 & -0.005 & 0.016 & -0.030 & -0.007 & -0.011 & -0.015 & -0.094 & 0.005 & 0.013 & -0.049 & -0.033 & -0.120 & -0.043 & -0.159 & -0.137 & -0.117 \\
\hline
\end{tabular}

$\begin{array}{clcl}\text { C } & \text { Consumo Privado } & \text { X } & \text { Exportaciones No Cobre } \\ \text { E } & \text { Tipo de Cambio Real } & \text { R } & \text { TPM } \\ \text { G } & \text { Gasto de Gobierno } & \text { PI } & \text { Inflación } \\ \text { INC } & \text { Inversión Privada No Cobre } & \text { PIBC } & \text { PIB Cobre } \\ \text { ENC } & \text { Empleo No Cobre } & \text { IC } & \text { Inversión Cobre } \\ \text { MNP } & \text { Importaciones No Petróleo } & \text { EC } & \text { Empleo Cobre } \\ \text { PIB } & \text { PIB } & \text { WC } & \text { Salarios Reales Cobre } \\ \text { W } & \text { Salarios Reales No Cobre } & \text { EE } & \text { Energía Eléctrica Cobre }\end{array}$

Fuente: Los autores basado en el modelo DSGE

Tabla 16: Impacto de un aumento de un $1 \%$ en la productividad de la minería.

\begin{tabular}{|c|c|c|c|c|c|c|c|c|c|c|c|c|c|c|c|c|}
\hline Años & C & $\mathrm{E}$ & G & INC & ENC & MNP & PIB & W & $X$ & $R$ & $\mathrm{PI}$ & PIBC & IC & $\mathrm{EC}$ & WC & $\mathrm{EE}$ \\
\hline 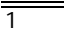 & 0.013 & -0.028 & 0.006 & -0.262 & 0.035 & 0.040 & 0.192 & -0.014 & -0.003 & 0.050 & 0.059 & 0.164 & 1.433 & 0.257 & 0.152 & 0.435 \\
\hline 2 & -0.009 & -0.031 & 0.011 & -0.076 & -0.002 & 0.004 & 0.027 & -0.010 & -0.013 & 0.096 & 0.073 & 0.106 & 0.298 & 0.081 & 0.100 & 0.128 \\
\hline 3 & -0.002 & -0.006 & 0.013 & 0.078 & 0.011 & 0.011 & 0.027 & -0.014 & -0.012 & 0.102 & 0.085 & 0.063 & -0.242 & 0.002 & 0.042 & 0.017 \\
\hline 4 & 0.000 & -0.001 & 0.014 & 0.115 & 0.007 & 0.000 & 0.012 & -0.008 & -0.008 & 0.089 & 0.074 & 0.028 & -0.468 & -0.030 & 0.007 & -0.026 \\
\hline 5 & 0.002 & 0.007 & 0.013 & 0.106 & 0.002 & -0.006 & -0.001 & -0.001 & -0.004 & 0.064 & 0.050 & 0.000 & -0.493 & -0.039 & -0.013 & -0.042 \\
\hline \multicolumn{17}{|c|}{ Acumulado } \\
\hline 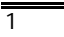 & 0.013 & -0.028 & 0.006 & -0.262 & 0.035 & 0.040 & 0.192 & -0.014 & -0.003 & 0.050 & 0.059 & 0.164 & 1.433 & 0.257 & 0.152 & 0.435 \\
\hline 5 & 0.003 & -0.058 & 0.057 & -0.041 & 0.053 & 0.050 & 0.258 & -0.046 & -0.040 & 0.402 & 0.341 & 0.361 & 0.516 & 0.270 & 0.289 & 0.513 \\
\hline 10 & 0.014 & -0.017 & 0.101 & 0.108 & 0.020 & 0.016 & 0.136 & -0.005 & -0.023 & 0.370 & 0.303 & 0.162 & -0.538 & 0.102 & 0.148 & 0.259 \\
\hline
\end{tabular}

$\begin{array}{clcl}\text { C } & \text { Consumo Privado } & \text { X } & \text { Exportaciones No Cobre } \\ \text { E } & \text { Tipo de Cambio Real } & \text { R } & \text { TPM } \\ \text { G } & \text { Gasto de Gobierno } & \text { PI } & \text { Inflación } \\ \text { INC } & \text { Inversión Privada No Cobre } & \text { PIBC } & \text { PIB Cobre } \\ \text { ENC } & \text { Empleo No Cobre } & \text { IC } & \text { Inversión Cobre } \\ \text { MNP } & \text { Importaciones No Petróleo } & \text { EC } & \text { Empleo Cobre } \\ \text { PIB } & \text { PIB } & \text { WC } & \text { Salarios Reales Cobre } \\ \text { W } & \text { Salarios Reales No Cobre } & \text { EE } & \text { Energía Eléctrica Cobre }\end{array}$




\section{Gráfico 9: Efecto del Precio del Cobre en el Crecimiento del PIB a una desviación estándar en el precio del cobre: Enclave vs Integrado.}

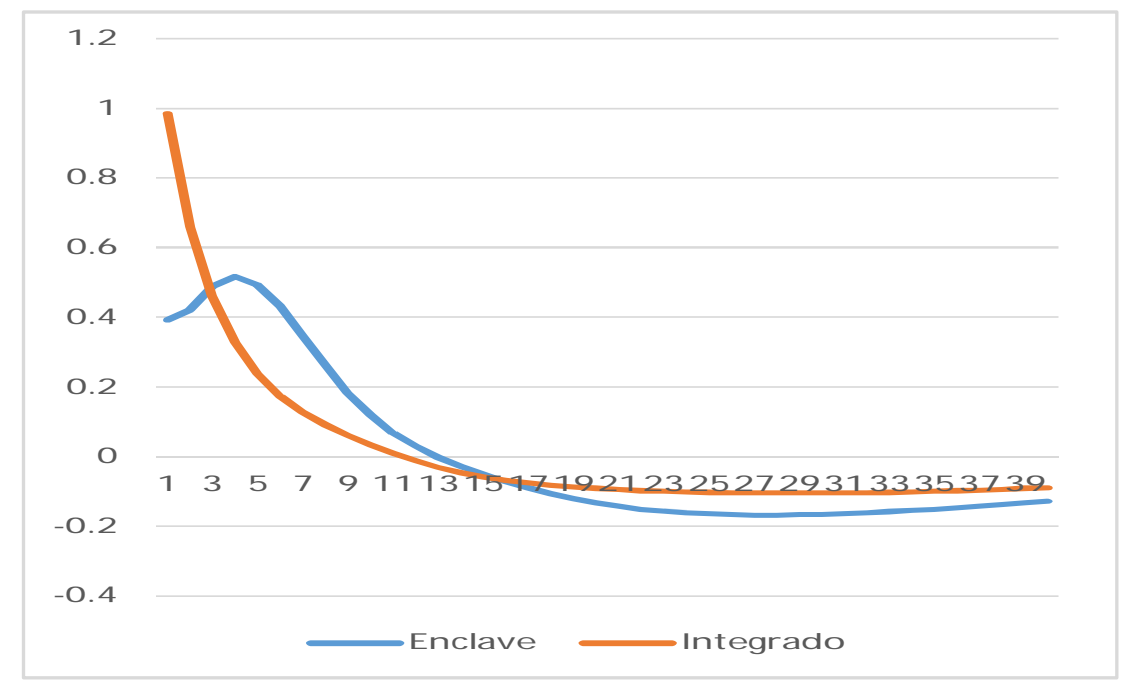

Fuente: Los autores basado en el modelo DSGE

En definitiva, el análisis de escenarios muestra los siguientes resultados:

- Un aumento sorpresivo del $1 \%$ en el precio de la energía en un trimestre, reduce el crecimiento del PIB en $0.027 \%$ durante el primer año24.

- Un aumento exógeno de los salarios del sector minero de un $1 \%$ en un trimestre produce una caída de $-0.139 \%$ del PIB durante el primer año. No obstante, si los salarios suben por la productividad este efecto contractivo no ocurre.

- Todos los efectos del estudio serían sustancialmente menores si erróneamente se considerara al sector minero como un enclave, es decir, como un sector sin conexiones con el resto de la economía. Lo anterior muestra la relevancia de la modelación empleada en el presente estudio, que incluye los efectos indirectos.

\footnotetext{
${ }^{24}$ Es importante aclarar que el efecto del precio eléctrico sólo se está midiendo su influencia en la economía a través del sector minero. Para visualizar el efecto global ver Agurto et al (2013).
} 


\section{CONCLUSIONES}

En este estudio se ha desarrollado y estimado un modelo DSGE para cuantificar el impacto del sector minero cobre en la economía Chilena. Las principales conclusiones son las siguientes:

- Al momento de modelar la relevancia de la minería cobre en la economía Chilena, es crucial reconocer las conexiones que tiene este sector con el resto de la economía, además de los recursos que genera para el Gobierno de Chile.

- Si bien el sector minero básicamente contribuye a través del precio del cobre, es importante reconocer que su producción depende de insumos que son en buena parte ofrecidos por el resto de la economía. Así, aumentos del precio del cobre gatillarán una serie de demandas que afectarán positivamente a muchos otros sectores de la economía.

- En términos cuantitativos, un aumento de un $1 \%$ del precio del cobre de una sola vez (con persistencia decreciente) causa un aumento acumulado de 0,16\% en el PIB en 5 años.

- En el período 2003-2013, el precio del cobre contribuyó en explicar en un 5,8\% la varianza el crecimiento del PIB de la economía. Porcentaje muy por encima de otros factores externos como el premio por riesgo, el crecimiento de los países desarrollados, la tasa de interés externa y el precio del petróleo.

- Si bien los aumentos del precio del cobre están asociados a caídas del tipo de cambio real, en el período 2003-2013 no hay evidencia que el precio del cobre explicara la varianza del tipo de cambio real. Por el contrario, esta variable dependen mayoritariamente de shocks el premio por riesgo y de la productividad, es decir, esta variable ha fluctuado por razones financieras y de productividad más que por cambios exógenos en los términos de intercambio. 
- Aumentos exógenos en el precio de la energía eléctrica, los salarios reales y la productividad minera tienen importantes efectos, no solo para el sector minero sino también para la economía como un todo. 


\section{Referencias Bibliográficas}

Acevedo, R. et al (2006). Matrices insumo-producto regionales. Estadística y economía, 7

(1).

Recuperado

de http:/ / www.ine.cl/ canales/ sala_prensa/ revistaseconomicas/ 25/ luisriffo25.pdf

Álvarez, R. et al (2004). Patrones de especialización y crecimiento en Chile. Banco Central de Chile, Documento de trabajo $\mathrm{N}^{\circ}$ 288. Recuperado desde http:/ / www researchgate.net/publication/28078189_Patrones_de_Especializacin_y_Cr ecimiento_Sectorial_en_Chile/ file/ 3 deec5231fc68578cc.pdf

An, S., Schorfheide, F. (2007). "Bayesian Analysis of DSGE Models", Econometric Reviews, Vol. 26(2), pp. 113-172.

Andres J, Burriel,P., Estrada, A., (2006), ' 'BEMOD: A DSGE Model for the Spanish Economy and the Rest of the Euro Area', Banco de Espana Documentos de Trabajo No 0631.

Arellano, J. (2012). El cobre como palanca de desarrollo para Chile. Centro de Estudios Públicos, $127 \quad$ (1). Recuperado desde http:/ / www.cepchile.cl/dms/archivo_5148_3298/ rev127_JPArellano.pdf

Aroca, P. (2000). Impacto de la Minería en la II Región. Dilemas y Debates en torno al Cobre (págs. 79-100). Santiago: Dolmen Ediciones.

Banco Central de Chile (2003). "Modelos macroeconómicos y proyecciones del Banco Central de Chile", Banco Central de Chile. Santiago, Chile.

Bems, R., de Carvalho Filho, I., (2011), The Current Account and Precautionary Savings for Exporters of Exhaustible Resources', Journal of International Economics, 84(1), pp 4864.

Bodenstein M, Erceg, C.J., Guerrieri, L. (2011), 'Oil Shocks and ExternalAdjustment', Journal of International Economics, 83(2), pp 168-184.

Bohn, H, Deacon, R.T. (2000), 'Ownership Risk, Investment, and the Use of Natural Resources', The American Economic Review, 90(3), pp 526-549.

Borregaard, N. (). Valorización económica de los impactos ambientales en la minería chilena. Ambiente y desarrollo. Recuperado de http:/ / www.cipma.cl/ web/ 200.75.6.169/ RAD/ 2001/___Borregaard.pdf

Caputo, R., Liendo, F., Médina, J.P., (2007), "New Keynesian Models for Chile in the InflationTargeting Period," Editado por Frederic Mishkin y Klaus Schmidt-Hebbel en "Monetary Policy under Inflation Targeting", Banco Central de Chile, Chile. 
Chen, Y., Rogoff, K, Rossi, B., (2010). "Can Exchange Rates Forecast Commodity Prices?," Quarterly Journal of Economics, Vol.125(3), pp.1145-1194.

Céspedes, L. F.; Fornero, J., Galí, J., (2010). "Non-Ricardian Aspects of Fiscal Policy in Chile", @Conferencia Anual del Banco Central de Chile: "Política Fiscal y Desempeño Macroeconómico", 21 y 22 de Octubre, Banco Central de Chile.

Christiano, L.; Eichenbaum,M., Evans,C., (2005). "Nominal Rigidities and the Dynamic Effects of a Shock to Monetary Policy", Journal of Political Economy, Vol.113(1), pp. 1-45.

Comisión Chilena del Cobre (2013). Minería en Chile: Impacto en regiones y desafíos para su desarrollo. Fundación Chile: Santiago, Chile. Recuperado desde http:/ / www.cochilco.cl/ descargas/ estadisticas/libro/ Libro_Mineria_en_Chile_Impacto_ en_Regiones_y_Desafios_para_su_Desarrollo.pdf

De Gregorio, J. (2009). El crecimiento en Chile y el cobre. Banco Central de Chile. Recuperado desde http:// www.bcch.cl/ politicas/ presentaciones/ consejeros/ pdf/ 2009/ jdg01092009.pdf

Dejong, D., Dave, C., (2011), Structural Econometrics, Second Edition, Princeton.

Dib, A., (2008), 'Welfare Effects of Commodity Price and Exchange Rate Volatilities in a Multi-Sector Small Open Economy Model', Bank of Canada Working Paper No 2008-8.

Erceg, C. J., Guerrieri, L., Gust, C., (2006). "SIGMA: A New Open Economy Model for Policy Analysis," International Journal of Central Banking, Vol. 2(1), pp. 1-50.

Galí, J., López-Salido,D., VallésJ., (2004). "Rule-of-Thumb Consumers and the Design of Interest Rate Rules”, Journal of Money, Credit and Banking, Vol. 36(4), pp. 739-763.

Galí, J. (2008). Monetary policy, inflation, and the business cycle: an introduction to the new Keynesian framework. Princeton University Press. Princeton, New Jersey.

Galí, J., Gertler, M. (2007). "Macroeconomic Modeling for Monetary Policy Evaluation", Journal of Economic Perspectives, Vol. 21(4), pages 25-45.

Galí, J.; López-Salido,D., Vallés,J., (2007). "Understanding the Effects of Government spending on Consumption," Journal of the European Economics Association, Vol. 5, pp. 227-270.

García, C.J., González, W., (2014), "Why Does Monetary Policy Respond to the Real Exchange Rate in Small Open Economies? A Bayesian Perspective," Empirical Economics, May.

García, C. J., González, P., Moncado, A., (2013), "Macroeconomic Forecasting in Chile: a Bayesian Structural Approximation, "Journal Economía Chilena (the Chilean Economy Journal), Central Bank of Chile, April.

Garcia, C.J., Restrepo, J., Roger, S., (2011a) "How much should inflation targeters care about the exchange rate?," Journal of International Money and Finance, 30, 1590-1617. 
Garcia, C.J., Restrepo J., Tanner, E., (2011b) "Fiscal Rules in a volatile world: a welfare-based approach," Journal of Policy Modeling, 33, 649-676.

García, C.J., Jaramillo, P., Selaive, J. (2007), "Stylized Facts of the International Business Cycle Relevant for the Chilean Economy Journal Economía Chilena (the Chilean Economy Journal), Central Bank of Chile, April.

Gertler, M., Kiyotaki,N., (2010). "Financial Intermediation and Credit Policy in Business Cycle Analysis", en B. Friedman y M. Woodford, Handbook of Monetary Economics, Vol. 3A, North Holland, Amsterdam.

Gross, I., Hansen, J., (2013) "Reserves of Natural Resources in a Small Open Economy," Research Discussion Paper, RDP 2013-14, Reserve Bank of Australia

Jääsleä, J, Nimark K., (2008), ' 'A Medium-Scale Open Economy Model of Australia', RBA Research Discussion Paper No 2008-07.

Jiménez, S. (2014). Actividad minera: Desafíos en energía. Serie informe económico, Libertad y Desarrollo, 236 (1). Recuperado desde http://www.lyd.org/wpcontent/ files_mf/sie236miner\%C3\%ADasusana.pdf

Lama, R, Medina, J.P. (2012), 'Is Exchange Rate Stabilization an Appropriate Cure for the Dutch Disease?', International Journal of Central Banking, 8(1), pp 5-46.

Laxton, D., Pesenti, P. (2003). "Monetary rules for small, open, emerging economies," Journal of Monetary Economics, Vol. 50(5), pp. 1109-1146.

Leal, J. (2000). Técnicas de valorización económica de impactos ambientales. Aplicabilidad y disponibilidad de información: El caso del sector minero. CIPMA. Recuperado de http:/ / intranet2.minem.gob.pe/ web/ archivos/ dgm/ publicaciones/ expedientes/ yanac ocha/ justificacion/ 8.4-

R1\%20T\%C3\%A9cnicas\%20de\%20Valorizaci\%C3\%B3n\%20Econ\%C3\%B3mica..pdf

Lees, K. (2009), 'Introducing KITT: The Reserve Bank of New Zealand New DSGE Model for Forecasting and Policy Design', Reserve Bank of New Zealand, Bulletin, 72(2), pp 5-20.

Leturia, F. et al (2004). Tributación y minería en Chile: antecedentes para un debate informado. Centro de Estudios Públicos. Recuperado desde http:// www.cepchile.cl/ dms/ archivo_3393_1681/r95_\%EE\%80\%80leturia\%EE\%80\% 81_merino_tributacionminera.pdf

Medina, J. P., Soto, C. (2006). "Model for Analysis and Simulations: A New DSGE for the Chilean Economy." Mimeo, Banco Central de Chile.

Medina, J. P., Soto, C., (2007). "The Chilean Business Cycles Through the Lens of a Stochastic General Equilibrium Model", Documento de Trabajo Nº 457, Banco Central de Chile. 
Meese, R., Rogoff, K., (1983). "Empirical Exchange Rate Models of the Seventies”, Journal of International Economics, Vol. 14(1), pp. 3-24.

Murchison, S., Rennison, A., (2006), ToTEM: The Bank of Canada's New," Quarterly Projection Model', Bank of Canada Technical Report No 97.

Natal, J-M., (2012), 'Monetary Policy Response to Oil Price Shocks', Journal of Money, Credit and Banking, 44(1), pp 53-101.

Restrepo, J., Soto, C., (2006), "Regularidades Empíricas de la Economía Chilena: 19862005,"Economía Chilena, 9, 163-185.

Servicio Nacional de Geología y Minería (2012). Anuario de la minería de Chile. SERNAGEOMIN, 205 páginas. Santiago, Chile. Recuperado desde http:/ / www.sernageomin.cl/pdf/ mineria/ estadisticas/ anuario/ anuario_2012.pdf

Smets, F., Wouters,R., (2003). “An Estimated Dynamic Stochastic General Equilibrium Model of the Euro Area", Journal of the European Economic Association, Vol. 1(5), pp. 11231175.

Smets, F., Wouters,R., (2007). "Shocks and Frictions in US Business Cycles: A Bayesian DSGE Approach", American Economic Review, Vol.97(3), pp. 586-606.

Soto, R. et al (1998). Una evaluación preliminar del impacto económico de El Teniente en la sexta región. ILADES - Georgetown University. Recuperado desde https:/ / plus.google.com/ url?sa=t\&rct=j\&q=\&esrc=s\&source=web\&cd=1\&cad=rja\&uact $=8 \&$ ved =0CCAQFjAA\&url=http\%3A\%2F\%2Fwww .researchgate.net\%2Fpublication\%2 F5115476_Una_Evaluacin_Preliminar_del_Impacto_Econmico_de_El Teniente_en la_sex ta_Regin\%2Ffile\%2Fd912f50a3499f163f0.pdf\&ei=72V3U_PoEeHIsASD-

4LADg\&usg=AFQjCNHbdCC6qCMMjVLAzN4O462E3R_ong\&sig2=o-MfPpoexaqsvNnXbqKpQ\&bvm=bv.66917471,d.cWc

Veroude, A. (2012), "The Role of Mining in an Australian Business Cycle Model," Paper presented at the 56th Australian Agricultural and Resource EconomicsSociety Annual Conference, Fremantle, 7-10 February.

Woodford, M. (2003). "Interest and Prices: foundations of a theory of monetary policy". Princeton University Press. Princeton, New Jersey. 


\section{Anexo $N^{\circ}$ 1: Modelo DSGE}

El modelo DSGE en términos generales está en las líneas propuestas por Christiano, Eichenbaum y Evans (2005) y Smets y Wouters (2003, 2007). Sin embargo, incorpora además del petróleo y el cobre, la energía eléctrica como insumos productivos.

\section{Hogares}

Hay un continuo de familias de tamaño unitario, indexadas por $i \in[0,1]$. En el modelo existen dos tipos de familias: una fracción $\left(1-\lambda_{c}\right)$ son las familias Ricardianas que tienen acceso al mercado de capitales y una fracción $\lambda_{c}$ son las familias restringidas, cuyos ingresos dependen únicamente de su salario laboral. Las preferencias de las familias Ricardianas están dadas por (A.1) donde $C_{t}^{o}$ es el consumo y $L_{t}^{o}$ es la oferta laboral de la familia:

$$
\max _{\left\{C_{t}^{o}(i), L_{t}^{o}(i), B_{t}^{o}(i), B_{t}^{o}(i)\right\}_{t=0}^{\infty}} E_{t} \sum_{t=0}^{\infty} \beta^{t}\left(\frac{\left(C_{t}^{o}(i)-h C_{t-1}^{o}(i)\right)^{1-\sigma}}{1-\sigma}-\frac{L_{t}^{o}(i)^{1+\rho_{L}}}{1+\rho_{L}}\right)
$$

El coeficiente $\sigma>0$ mide la aversión al riesgo y $\rho_{L}$ mide la des-utilidad de trabajar, el inverso de este parámetro es también el inverso es la elasticidad de las horas trabajadas al salario real. $h$ mide la formación de hábito para capturar la dinámica del consumo.

La restricción presupuestaria, explicada, en detalle en la Sección 3, de las familias no restringidas está dada por:

$$
\begin{gathered}
\left(1+t_{c}\right) P_{t} C_{t}^{o}(i) \leq\left(1-t_{w}\right) W_{t}(i) L_{t}^{o}(i)+B_{t}^{o}(i)-S X_{t} B_{t}^{o^{*}}(i)+D_{t}^{o} \\
-R_{t}^{-1} B_{t+1}^{o}(i)+\left(\Phi\left(\frac{B_{t+1}^{o *}}{P I B_{t}}\right) R_{t}^{*}\right)^{-1} B_{t+1}^{o^{*}}(i)
\end{gathered}
$$

Las familias restringidas están sujetas a la siguiente restricción presupuestaria (exenta de impuestos a los ingresos):

$$
\left(1+t_{c}\right) P_{t} C_{t}^{R}(i)=W_{t}(i) L_{t}^{R}(i)
$$

\section{Intermediarios Financieros}

Los intermediarios financieros prestan fondos $S_{j t}$ obtenidos de las familias a las firmas no-financieras. Estos fondos provienen de la riqueza propia $N_{j t}$ y los fondos obtenidos de las familias $B_{j t}$.

$$
Q_{t} S_{j t}=N_{j t}+B_{j t}
$$

La riqueza financiera evoluciona por el spread entre la tasa de mercado $R_{F t+1}$ que tienen los productores de capital y la de política monetaria $R_{t+1}$, que también es la efectiva para las familias.

$$
N_{j t+1}=\left(R_{F t+1}-R_{t+1}\right) Q_{t} S_{j t}+R_{t+1} N_{j t}
$$

El objetivo del intermediario financiero es maximizar su riqueza esperada, dada por:

$$
V_{j t}=\max _{\left\{N_{j t+i+1}\right\}_{i=0}^{\infty}} E_{t} \sum_{i=0}^{\infty}(1-\theta) \theta^{i} \beta^{i} \Lambda_{t, t+1+i}\left[\left(R_{F t+1+i}-R_{t+1+i}\right) Q_{t+i} S_{j t+i}+R_{t+1+i} N_{j t+i}\right]
$$

Gertler y Karadi (2009) introducen riesgo moral al problema (A.6), demostrando que en términos agregados: 


$$
Q_{t} S_{t}=\phi_{t} N_{t}
$$

La ecuación (A7) indica que la disponibilidad total de crédito privado es la riqueza de los intermediarios multiplicada por un factor $\phi$ que indica el grado de aplacamiento de los intermediarios.

\section{Firmas de Bienes Intermedios}

Las firmas de bienes intermedios utilizan capital $K_{t}$, trabajo $L_{t}$, y bienes importados $M_{t}$ para producir bienes intermedios $Y_{t}$.Al final del período $t$, las firmas productoras de bienes intermedios compran capital $K_{t+1}$ para utilizarlo en la producción en el período siguiente. Después de finalizado el proceso productivo, las firmas tienen la opción de vender el capital. Para adquirir los recursos que financian la compra del capital, la firma entrega $S_{t}$ derechos iguales al número de unidades de capital adquiridas $K_{t+1} \mathrm{y}$ el precio de cada derecho es $Q_{t}$. Esto es, $Q_{t} K_{t+1}$ es el valor del capital adquirido y $Q_{t} S_{t}$ es el valor de los derechos contra capital. Luego, se debe satisfacer:

$$
Q_{t} K_{t+1}=Q_{t} S_{t}
$$

En cada tiempo t, la firma produce, $Y_{t}$ usando capital, trabajo, bienes importados. Sea $A_{t}$ la productividad total de factores. Luego, la producción está dada por:

$$
Y_{t}=A_{t} K_{t}^{\beta} L_{t}^{\alpha} M_{t}^{1-\alpha-\beta}
$$

Sea $P_{m, t+k}$ el precio del bien intermedio. Dado que la decisión de la firma está hecha al final de periodo t, el problema de maximización de la firma que produce bienes intermedios es:

$$
\begin{aligned}
& \max _{\left\{K_{t+k}(j), L_{t+k}(j), M_{t+k}(j)\right\}_{k=0}^{\infty}} \sum_{k=0}^{\infty} \beta^{k} \Lambda_{t, t+k} E_{t}\left\{\left(P_{m, t+k} Y_{t+k}(j)+(1-\delta) K_{t+k}(j) Q_{t+k}\right)\left(1-t_{t}^{u}\right)\right\} \\
& -\sum_{k=0}^{\infty} \beta^{k} \Lambda_{t, t+k} E_{t}\left\{\left(R_{F, t+k} Q_{t+k-1} K_{t+k}(j)+W_{t+k} L_{t+k}(j)+S X_{t+k} M_{t+k}(j)\right)\left(1-t_{t}^{u}\right)\right\}
\end{aligned}
$$

Los impuestos a los beneficios $t_{t}^{u}$ de estas empresas no tienen efectos en la demandas por insumos ni tampoco efectos fiscales por el supuesto de competencia perfecta en la producción de estos bienes que impone beneficios ceros.

\section{Firmas Productoras de Capital}

Las firmas productoras de capital compran capital de las firmas productoras de bienes intermedios, reparan el capital depreciado, y construyen nuevo capital con el capital reparado. Si definimos a $I_{t}$ como la inversión, el problema de maximización de las firmas productoras de capital es:

$$
\max _{\left\{I_{\tau}\right\}_{\tau=t}^{\infty}} \sum_{\tau=t}^{\infty} \beta^{\tau} \Lambda_{t, \tau} E_{t}\left\{\left(\left(Q_{\tau}-1\right) I_{\tau}-t_{t}^{u} Q_{\tau} I_{\tau}-f\left(\frac{I_{\tau}}{I_{\tau-1}}\right) I_{\tau}\right)\right\}
$$

En otras palabras, la firma productora de bienes de capital obtiene una ganancia por invertir en cada período de $\left(Q_{\tau}-1\right) I_{\tau}$ menos los costos de ajuste $f\left(I_{\tau} / I_{\tau-1}\right)$. Por último $t u$ son impuestos a las ganancias. La ley de movimiento del capital es dada por:

$$
K_{t-1}=(1-\delta) K_{t}+I_{t}
$$

\section{Firmas de Retail}

El producto final $Y_{t}$ se obtiene agregando (a través de una función $\mathrm{CES}$ ) la producción de firmas intermedias. Suponemos que esto se hace por parte de otras firmas, que llamamos de retail y que simplemente empacan la producción de bienes intermedios: 


$$
Y_{t}=\left(\int_{0}^{1} Y_{f t}^{\frac{\varepsilon-1}{\varepsilon}} d f\right)^{\frac{\varepsilon}{\varepsilon-1}}
$$

Como en Christiano, Eichenbaum y Evans (2005) las firmas de retail enfrentan precios à la Calvo e indexación parcial. Luego, el problema de maximización para una firma de retail j está dado por:

$$
\begin{aligned}
& \max _{\left\{P_{t}^{*}(j)\right\}_{k=0}^{\infty}} \sum_{k=0}^{\infty} \theta^{k} E_{t}\left\{\beta^{k} \Lambda_{t, t+k} Y_{t+k}(j)\left(P_{t}^{*}(j) \prod_{l=1}^{k}\left(\pi_{t+l-1}^{k}\right)^{\delta_{D}}-M C_{t+k}\right)\right\} \\
& \text { s.a. } \quad Y_{t+k}(j) \leq\left(\frac{P_{t}^{*}(j)}{P_{t+k}}\right)^{-\varepsilon_{D}} Y_{t+k}
\end{aligned}
$$

Donde $M C_{t+k}$ son los costos marginales de la empresa de retail. En particular, en cada período una firma está dispuesta a ajustar sus precios con probabilidad $(1-\theta)$. Entre esos períodos, la firma está dispuesta a indexar parcialmente (es decir, $\delta_{D} \in[0,1]$ ) su precio a la tasa de inflación pasada. Con estos supuestos, el nivel de precios evoluciona de acuerdo a

$$
P_{t}=\left((1-\theta)\left(P_{t}^{*}\right)^{\frac{1}{1-\varepsilon}}+\theta\left(\pi_{t-1}^{\delta_{D}} P_{t-1}\right)^{\frac{1}{1-\varepsilon}}\right)^{1-\varepsilon}
$$

Suponemos que el producto final que es usado por consumidores y firmas es una combinación entre $Y_{t} \mathrm{e}$ importaciones de petróleo para transporte TOIL $_{t}$.

\section{Política Monetaria}

La política monetaria sigue una regla de Taylor que responde ante cambios en el producto, la inflación y el tipo de cambio.

$$
\begin{gathered}
R_{t}^{*}=\bar{R}\left(\left(\frac{\Pi_{t+1}}{\bar{\Pi}}\right)^{\phi_{\pi}}\left(\frac{P I B_{t}}{\overline{P I B}}\right)^{\phi_{y}}\left(\frac{E_{t}}{\bar{E}}\right)^{\zeta_{e}^{1}}\left(\frac{E_{t}}{E_{t-1}}\right)^{\zeta_{e}^{2}}\right) e^{u_{t}^{R}} \\
R_{t}=\left(R_{t-1}\right)^{\Omega_{R}}\left(R_{t}^{*}\right)^{1-\Omega_{R}}
\end{gathered}
$$

Donde $\bar{R}$ es la tasa natural, $\Pi_{t}$ es la inflación total, $\bar{\Pi}$ es el objetivo inflacionario, $\overline{P I B}$ es el PIB potencial, $E_{t}$ es el tipo de cambio real, $\bar{E}$ es el tipo de cambio real de equilibrio y $u_{t}^{R}$ es un shock monetario. En las estimaciones de las ecuaciones (A16) y (A17) se utilizó el PIB sin recursos naturales (es decir, sin sector cobre).

\section{Exportaciones no mineras}

En el modelo, se supone que las exportaciones $X_{t}$ dependen del tipo de cambio real $E_{t}$, de la actividad económica internacional $P I B_{t}^{*}$ y además, presenta cierto grado de inercia $\Omega$. Luego,

$$
\begin{gathered}
X_{t}=\left(E_{t}\right)^{-\eta^{*}} P I B_{t}^{*} \\
X_{t}=\left(X_{t-1}\right)^{\Omega}\left(X_{t}\right)^{1-\Omega}
\end{gathered}
$$

\section{Riesgo País}

Además, como en Schmitt-Grohé y Uribe (2003), para cerrar el modelo se supone que el riesgo país depende de la deuda externa de la siguiente forma: 


$$
S X_{t}\left(\Phi\left(\frac{B_{t+1}^{*}}{P I B_{t}}\right) R_{t}^{*}\right)^{-1}
$$

\section{Restricción de Recursos, Producción de Cobre y Gasto de Gobierno}

La regla fiscal establece que el gasto depende los ingresos estructurales $I T$, más un ajuste por exceso de deuda pública. En otras palabras si esta deuda es consistente con su valor de largo plazo $B^{G^{*}}$, entonces el valor del gasto fiscal es igual a los ingresos estructurales $I T$.

$$
P_{t} G_{t}=\left(\frac{B_{t}^{G^{*}}}{B^{G^{*}}}\right)^{-\phi^{G}} I T
$$

La restricción presupuestaria del gobierno, que incluye todos los ingresos de los impuestos más las transferencias del cobre $\gamma^{c u} S X_{t} P_{t}^{c u} Q C U_{t}$ es:

$$
t c P_{t} C_{t}+t_{t}^{u} P_{t} I_{t}+t_{t}^{u} P_{t} I_{t}^{c u}+t w W_{t} N_{t}+\left(R_{t}\right)^{-1} B_{t+1}^{G}+\gamma^{c u} S X_{t} P_{t}^{c u} Q C U_{t}=B_{t}^{G}+P_{t} G_{t}
$$

Donde $B_{t}^{G}$ es la deuda del gobierno y $\gamma^{c u}$ es el porcentaje del valor total de las exportaciones del cobre que son del gobierno (CODELCO). 


\section{Anexo $N^{\circ}$ 2: Datos Utilizados}

La muestra del estudio es trimestral desde 2003.I al 2013.IV.

\subsection{Datos Macroeconómicos.}

La información macroeconómica fue tomada de la página WEB del Banco Central de Chile: http:// si3.bcentral.cl/ Siete/ secure/ cuadros/ home.aspx

\subsection{Datos Sector Minero.}

\section{PIB Cobre:}

BCCh (trimestral, 2003-2013, empalme con series de referencia 2008)

http://si3.bcentral.cl/Siete/secure/cuadros/home.aspx

\section{Inversión Minera:}

Cochilco, Inversión Gran Minería del Cobre, Anuario (dólares de cada año, anual 2003-2013, calculado en términos reales al multiplicarse por tipo de cambio dividido por deflactor de la inversión.

\section{http://www.cochilco.cl/estadisticas/anuario.asp}

BCCh Formación bruta de capital físico (FBCF), página WEB (pesos de cada año, empalme con series de referencia 2008, anual 2003-2013, calculado en términos reales al dividirse por deflactor de la inversión).

\section{http://si3.bcentral.cl/Siete/secure/cuadros/home.aspx}

Con esta información se obtiene participación anual de la inversión minera sobre inversión total.

Dentro de un año, se supone que la participación de la inversión minera aumenta linealmente para alcanzar participación anual.

BCCh FBCF (trimestral 2003-2013, empalme con series de referencia 2008) página WEB http://si3.bcentral.cl/Siete/secure/cuadros/home.aspx

\section{Empleo minero:}

INE, ocupados por categoría, minería y canteras, 2013-2010: 
http://www.ine.cl/canales/chile estadistico/mercado del trabajo/empleo/series estadisti cas/nuevas empalmadas/series fecha.php

INE, ocupados por categoría, minería y canteras, 2010-2013:

http://www.ine.cl/canales/chile estadistico/mercado del trabajo/nene/series trimestrale s 2011.php

Las series luego fueron empalmadas y trimestralizada (Microsoft Excel @) y desestacionalizada por el programa Census X-13 de Eviews 8.0 $\odot$.

\section{Salario Minero:}

Índice nominal de remuneraciones, explotación de minas y canteras, INE (series obtenida de dos series: D) históricas empalmadas 1993 - 2007 (base enero 2006=100) y B) series referenciales base anual $2009=100)$ ).

http://www.ine.cl/canales/chile estadistico/mercado del trabajo/remuneraciones/series estadisticas/nuevo series estadisticas.php

La serie es mensual, luego fue trimestralizada (Microsoft Excel @) y desestacionalizada por el programa Census X-13 de Eviews 8.0 ㅇ.

\section{Energía Eléctrica}

Cochilco Consumo Nacional de la Energía en la Minería del Cobre, Anuario (promedio anual, 20032013, en TJ).

http://www.cochilco.cl/estadisticas/energia.asp

La serie fue transformada en GWH ( $1 \mathrm{GWh} \approx 0.28 \mathrm{TJ})$

http://www.Ingplants.com/conversiontables.html

CNE Generación Bruta SIC - SING (promedio anual en GWH)

http://www.cne.cl/estadisticas/energia/electricidad

Con esta información se obtiene la participación anual del consumo de energía eléctrica minera sobre el consumo nacional total.

CNE Generación Bruta SIC - SING (promedio mensual en GWH). Fue trimestralizada (Microsoft Excel () y desestacionalizada por el programa Census X-13 de Eviews 8.0 ๑.

http://www.cne.cl/estadisticas/energia/electricidad

Dentro de un año, se supone que la participación del consumo eléctrico minero aumenta linealmente para alcanzar participación anual. 


\section{Precio de la Energía Eléctrica}

El precio de la energía relevante para la minería se construye con el promedio ponderado entre el precio SICy SING.

Se usa como ponderadores el porcentaje del PIB minero de las regiones I y II para el SIGN $(0,6)$ y el porcentaje del PIB minero de las regiones III, IV, V y VI para el SIC $(0,4)$. No obstante la información regional solo se tiene información para el 2010-2012.

Los detalles sobre los precios es el siguiente:

- Desde el tercer trimestre del año 2006 hasta el cuarto trimestre del año 2013, los precios corresponden al "Precio Promedio de Mercado de Clientes no Sometidos a Regulación de Precios - en \$/ Kwh", publicado por la Comisión Nacional de Energía. AL respecto, cabe señalar que dado que los precios publicados están en promedios de cuatro meses, para un trimestre cualquiera se ha usado el promedio simple entre los dos cuatrimestres que incluyen los tres meses del trimestre correspondiente (por ejemplo, para el tercer trimestre del año 2006, se ha calculado el promedio simple entre el cuatrimestre "Junio 2006 - Septiembre 2006" y el cuatrimestre "Julio 2006 - Octubre 2006".

- Para el período comprendido entre el primer trimestre del año 2000 y el segundo trimestre del año 2006, se han usado los datos calculados por SYNEX, usados en el estudio "Impacto Macroeconómico del Retraso en las Inversiones de Generación Eléctrica en Chile", Renato Agurto, Fernando Fuentes H., Carlos J. García, y Esteban Skoknic; Serie Documentos de Investigación I - 288, Universidad Alberto Hurtado (2013). 


\section{Anexo N³: Convergencia y Parámetros Estimados del Modelo DSGE}

\section{Gráfico B.1: Convergencia y Estabilidad de los Parámetros}
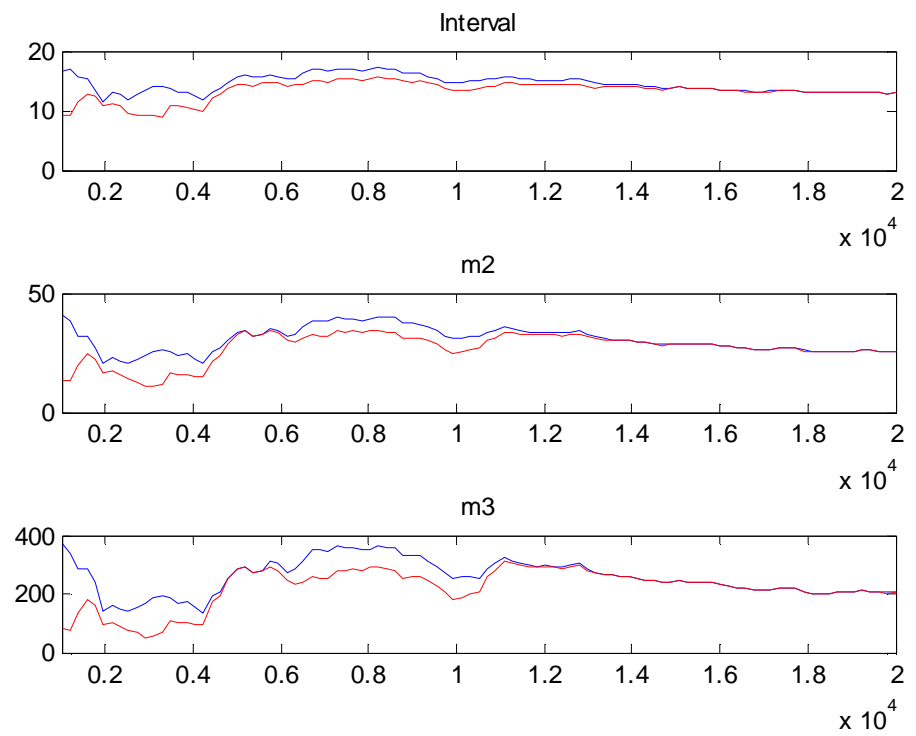

Fuente: Simulaciones realizadas por los autores en base al Modelo DSGE 
Tabla B.1: Parámetros Estimados del Modelo DSGE

\begin{tabular}{|c|c|c|c|c|c|c|}
\hline Parámetros & Prior & Posterior & Intervalo de C & $90 \%$ & Distribución Prior & Desviación Estándar \\
\hline sigma & 2 & 1.7726 & 1.6361 & 1.8897 & gamma & 0.1 \\
\hline $\mathrm{h}$ & 0.3 & 0.2638 & 0.2018 & 0.3359 & beta & 0.05 \\
\hline rho_L & 1 & 1.1488 & 1.0025 & 1.2985 & gamma & 0.1 \\
\hline rho_G & 0.9 & 0.9119 & 0.8497 & 0.9793 & beta & 0.05 \\
\hline rho_A & 0.9 & 0.931 & 0.9051 & 0.9535 & beta & 0.05 \\
\hline rho_Rstart & 0.9 & 0.6335 & 0.5437 & 0.7146 & beta & 0.05 \\
\hline rho_Ystart & 0.9 & 0.974 & 0.9651 & 0.9827 & beta & 0.05 \\
\hline rho_Oil & 0.9 & 0.8655 & 0.7959 & 0.9337 & beta & 0.05 \\
\hline rho_Pcu & 0.9 & 0.8623 & 0.8377 & 0.8915 & beta & 0.05 \\
\hline rho_GD & 0.1 & 0.0098 & 0.0083 & 0.0111 & beta & 0.05 \\
\hline index & 0.906 & 0.8815 & 0.8013 & 0.9641 & beta & 0.05 \\
\hline xi & 0.804 & 0.8173 & 0.8024 & 0.8298 & beta & 0.01 \\
\hline index_w & 0.9 & 0.6731 & 0.5775 & 0.785 & beta & 0.05 \\
\hline xi_w ${ }^{-}$ & 0.67 & 0.8961 & 0.8802 & 0.9136 & beta & 0.05 \\
\hline beta1 & 0.8 & 0.7961 & 0.7821 & 0.8106 & gamma & 0.01 \\
\hline beta2 & 0.1 & 0.0999 & 0.0987 & 0.1013 & beta & 0.001 \\
\hline rho_R & 0.92 & 0.9201 & 0.906 & 0.9325 & beta & 0.01 \\
\hline rho_inf & 2 & 1.9894 & 1.8261 & 2.1729 & beta & 0.1 \\
\hline rho_y & 0.5 & 0.5758 & 0.456 & 0.7018 & beta & 0.1 \\
\hline rho_e1 & 0.3 & 0.2655 & 0.1338 & 0.3943 & beta & 0.2 \\
\hline rho_e2 & 0.3 & 0.0811 & 0.0001 & 0.1715 & beta & 0.2 \\
\hline rho_E & 0.3 & 0.3015 & 0.2848 & 0.3198 & beta & 0.01 \\
\hline pmg_M & 0.5 & 0.3838 & 0.281 & 0.4713 & beta & 0.1 \\
\hline pmg_L & 0.5 & 0.1662 & 0.1344 & 0.193 & beta & 0.1 \\
\hline pmg_K & 0.5 & 0.5271 & 0.3736 & 0.6914 & beta & 0.1 \\
\hline theta_TOIL & 0.5 & 0.4599 & 0.2992 & 0.6423 & beta & 0.1 \\
\hline theta_L & 0.5 & 0.8001 & 0.7209 & 0.8725 & beta & 0.1 \\
\hline theta_K $\mathrm{K}$ & 0.5 & 0.5046 & 0.3333 & 0.6619 & beta & 0.1 \\
\hline theta_M & 0.5 & 0.5602 & 0.419 & 0.688 & beta & 0.1 \\
\hline pmg_TOTOL & 0.1 & 0.0694 & 0.0103 & 0.1306 & beta & 0.05 \\
\hline pmg_G & 0.5 & 0.5863 & 0.5159 & 0.6519 & beta & 0.05 \\
\hline trend_PIB & 1.1 & 1.2909 & 1.2053 & 1.3755 & gamma & 0.1 \\
\hline trend_Oil & 2.42 & 2.4448 & 2.3135 & 2.5874 & gamma & 0.1 \\
\hline trend_Pcu & 3.28 & 3.2443 & 3.0929 & 3.3895 & gamma & 0.1 \\
\hline trend_PIBstar & 1.22 & 1.2056 & 1.0516 & 1.3425 & gamma & 0.1 \\
\hline trend_L $\mathrm{L}$ & 0.71 & 0.5789 & 0.4701 & 0.6934 & gamma & 0.1 \\
\hline trend_E & 0.5 & 0.112 & 0.0006 & 0.228 & unif & 0.2887 \\
\hline constänte_R & 0.99 & 0.9837 & 0.828 & 1.1513 & gamma & 0.1 \\
\hline constante_PI & 0.75 & 0.7538 & 0.6067 & 0.8976 & gamma & 0.1 \\
\hline constante_Rstar & 0.5 & 0.3869 & 0.0004 & 0.728 & unif & 0.2887 \\
\hline rho_PEE & 0.5 & 0.8518 & 0.8016 & 0.9022 & beta & 0.1 \\
\hline pmḡ_EE_EN_COBRE & 0.5 & 0.2564 & 0.1317 & 0.3676 & beta & 0.1 \\
\hline index_w_COBRE & 0.9 & 0.9046 & 0.8335 & 0.9712 & beta & 0.05 \\
\hline xi_w_COB̈RE & 0.67 & 0.6216 & 0.5705 & 0.6813 & beta & 0.05 \\
\hline pmg_EN_COBRE & 0.5 & 0.1423 & 0.0555 & 0.2258 & beta & 0.1 \\
\hline pmg_L_C̄OBRE & 0.5 & 0.0848 & 0.051 & 0.1188 & beta & 0.1 \\
\hline pmg_K_COBRE & 0.5 & 0.5461 & 0.4031 & 0.6778 & beta & 0.1 \\
\hline rho_A_COBRE & 0.9 & 0.9045 & 0.8917 & 0.9168 & beta & 0.01 \\
\hline trend_PIB_COBRE & 0.1 & 0.1039 & 0.09 & 0.1187 & gamma & 0.01 \\
\hline trend_PEE & 0.64 & 0.6381 & 0.4976 & 0.7886 & gamma & 0.1 \\
\hline
\end{tabular}

Fuente: Los autores basado en el modelo DSGE 
Tabla B.2: Desviaciones Estándar Shocks Estimados del Modelo DSGE

\begin{tabular}{l|cccccc} 
Desviaciones Estándar Shocks & Prior & Posterior & Intervalo de Confianza 90\% & Distribución Prior & Desviación Estándar \\
\hline Err_C & 1.08 & 0.4898 & 0.3557 & 0.6311 & invg2 & 0.5 \\
Err_E & 3.56 & 5.1476 & 4.5078 & 5.7673 & invg2 & 0.5 \\
Err_G & 1.72 & 1.1 & 0.9047 & 1.2844 & invg2 & 0.5 \\
Err_M & 4.78 & 4.5151 & 3.9697 & 5.1656 & invg2 & 0.5 \\
Err_Oil & 16.07 & 16.2808 & 15.608 & 17.0067 & invg2 & 0.5 \\
Err_Pcu & 16.53 & 16.627 & 15.8637 & 17.4668 & invg2 & 0.5 \\
Err_PIB & 1.16 & 1.8313 & 1.3863 & 2.2744 & invg2 & 0.5 \\
Err_Q & 9.76 & 9.7315 & 8.9636 & 10.7295 & invg2 & 0.5 \\
Err_W & 0.87 & 0.4109 & 0.3251 & 0.4877 & invg2 & 0.5 \\
Err_X & 5.03 & 5.1674 & 4.5989 & 5.7607 & invg2 & 0.5 \\
Err_Ystart & 2.81 & 3.31 & 2.662 & 3.9391 & invg2 & 0.5 \\
Err_R & 0.48 & 0.2178 & 0.1732 & 0.2618 & invg2 & 0.5 \\
Err_PI & 0.96 & 0.7168 & 0.5913 & 0.8459 & invg2 & 0.5 \\
Err_Rstart & 0.89 & 0.9244 & 0.7517 & 1.0735 & invg2 & 0.5 \\
Err_QCU & 3.59 & 3.7614 & 3.2334 & 4.2889 & invg2 & 0.5 \\
Err_COBRE_I & 6.46 & 6.4473 & 5.8375 & 7.0691 & invg2 & 0.5 \\
Err_I & 4.6 & 4.4445 & 3.9205 & 4.93 & invg2 & 0.5 \\
Err_L_COBRE & 1.4 & 4.4021 & 3.7446 & 5.0181 & invg2 & 0.5 \\
Err_L & 0.81 & 1.5259 & 1.2768 & 1.753 & invg2 & 0.5 \\
Err_COBRE_W & 0.9 & 0.5559 & 0.4213 & 0.6845 & invg2 & 0.5 \\
Err_EE_COBRE & 4.34 & 5.1935 & 4.3792 & 5.8902 & invg2 & 0.5 \\
Err_PEE & 6.84 & 6.9106 & 6.2696 & 7.6251 & invg2 & 0.5 \\
Err_A & 1.16 & 0.7197 & 0.5738 & 0.8635 & invg2 & 0.5 \\
Err_A_COBRE & 3.59 & 7.2804 & 5.834 & 8.6681 & invg2 & 0.5
\end{tabular}

Fuente: Los autores basado en el modelo DSGE 


\section{Anexo $N^{\circ}$ 4: Calibración de los Parámetros funciones Cobb-Douglas}

Las funciones que representa la producción de cobre son las siguientes (repetimos las ecuaciones del texto principal)

$$
\begin{aligned}
& Q C U_{t}=A_{t}^{C U} L_{t}^{C U \alpha} K_{t}^{C U \beta} E_{t}^{1-\alpha-\beta} \\
& E_{t}=O I L_{t}^{\delta} E E_{t}^{1-\delta}
\end{aligned}
$$

El cálculo de $\beta$ en la ecuación (1.6), se estima de la siguiente manera. Primero se obtiene la razón promedio Inversión Minería Cobre sobre el PIB minero. Segundo, se calcula la participación ó el $\beta$ por:

$$
\beta=\text { participación }=(r+\delta) \frac{K}{P I B}=(0.02368+0.02) * 0.23=0.51
$$

La tasa de interés se calcula suponiendo una tasa de descuento subjetiva de 0.9865, más un spread de 1\%. La tasa de depreciación $\delta$ es supuesta de $2 \%$ trimestral, tasa que es la misma tasa de depreciación para el resto de la economía, que a su vez fue fijada en ese valor para obtener valores razonables del estado estacionario (consumo sobre PIB, inversión sobre PIB, etc.).

En el caso de la energía, usamos los consumos de energía eléctrica como de combustible medios en TJ anuales que publica COCHILCO. Luego los consumos anuales en TJ son transformadas en unidades equivalentes de barriles de petróleo (se divide por 5.75/1000) y GWh (se multiplica por 0.28), puesto que en estas unidades existen precios en pesos (el precio del barril de petróleo es transformado en pesos al ser multiplicado por el tipo de cambio observado).

Los valores totales para cada tipo de energía son calculados por la multiplicación de los precios por la cantidad de barriles y de GWh, respectivamente. En seguida se obtienen las participaciones respectivas dividendo los valores anuales por el PIB del cobre en pesos de cada año. Así, se toma el promedio de las participaciones del período 2003-2013. En resumen, la energía total participa en un 10\% en el PIB cobre, 3\% combustible y 7\% energía eléctrica, con lo cual un 30\% del gasto total de energía es combustible y 70\% energía eléctrica.

La participación del trabajo se obtiene como residuo, una vez calculado la participación del capital y la energía $(1-0.51-0.1=0.39)$. 